\title{
Ru(II)- or Rh(III)-Catalyzed Difunctionalization of Alkenes by Tandem Cyclization of $N$-Aryl Acrylamides with Alkenes
}

Ramasamy Manoharan, Ravichandran Logeswaran, and Masilamani Jeganmohan*

Indian Institute of Technology Madras, Chennai 600036, Tamil Nadu, India

Email: mjeganmohan@iitm.ac.in

Supporting Information (SI)

Table of Contents

S2 - S8 Mechanistic studies

S9-S11 Single crystal X-Ray Studies

S12-S83 Copies of $\mathrm{H}^{1}, \mathrm{C}^{13}$ and DEPT135 


\section{Mechanistic Studies}

\section{Competition Experiments:}

\section{General Procedure for Competition reaction between amides}

A $15 \mathrm{~mL}$ schlenk tube with septum containing $\left[\mathrm{RuCl}_{2}\right.$ (p-cymene $\left.)\right]_{2}(5.0 \mathrm{~mol} \%), \mathrm{N}$ arylacrylamide1d $(0.5 \mathrm{mmol}, 1$ equiv), $N$-arylacrylamide amide $\mathbf{1 h}(0.5 \mathrm{mmol}, 1$ equiv) maleimide $2 \mathrm{a}(0.5 \mathrm{mmol}, 1 \mathrm{eq}), \mathrm{Cu}(\mathrm{OAc})_{2} \cdot \mathrm{H}_{2} \mathrm{O}$ (2.2 equiv) and $\mathrm{AgSbF}_{6}(20 \mathrm{~mol} \%)$ was evacuated and purged with nitrogen gas three times ( $\mathrm{AgSbF}_{6}$ was taken inside the glove box). To the tube were then added $\mathrm{ClCH}_{2} \mathrm{CH}_{2} \mathrm{Cl}(3.0 \mathrm{~mL}, 0.16 \mathrm{M})$ via syringe, after that the reaction mixture was evacuated and purged with nitrogen gas three times. After that, the septum was taken out immediately and a screw cap was used to cover the tube under the nitrogen atmosphere and again the reaction mixture stirred at room temperature for 5 minutes. Then, the reaction mixture was allowed to stir at $100{ }^{\circ} \mathrm{C}$ for $16 \mathrm{~h}$ in oil bath. After cooling to ambient temperature, the reaction mixture was diluted with $\mathrm{CH}_{2} \mathrm{Cl}_{2}$, filtered through Celite and the filtrate was concentrated. The crude residue was purified through a silica gel column using hexanes and ethyl acetate as eluent to give pure product 3dain21\% yield.
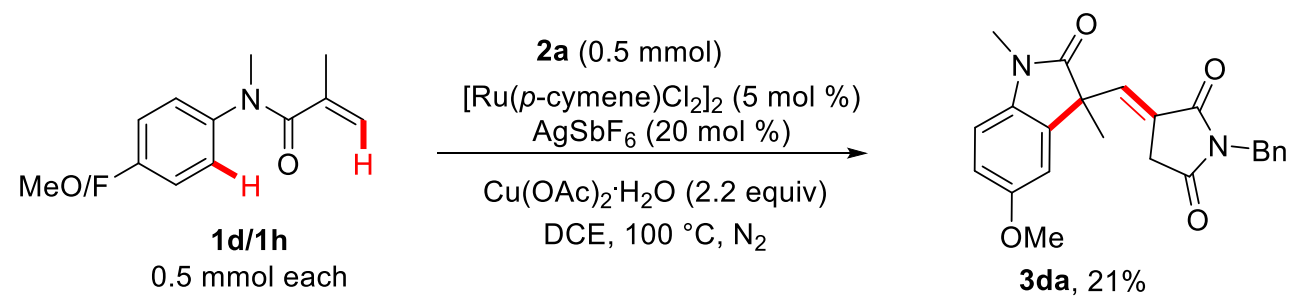

\section{Procedure for the preparation of uncyclized product 6:}

A $15 \mathrm{~mL}$ schlenk tube with septum containing $\left[\mathrm{RuCl}_{2} \text { (p-cymene) }\right]_{2}$ or $\left[\mathrm{Cp}^{*} \mathrm{RhCl}_{2}\right]_{2}(5.0 \mathrm{~mol}$ $\%$ ), $\mathrm{N}$-arylacrylamide 1a (50 mg, 1 equiv), maleimide 2a (1.2 eq), $\mathrm{CH}_{3} \mathrm{COOH}$ (4 equiv) and $\mathrm{AgSbF}_{6}(20 \mathrm{~mol} \%)$ was evacuated and purged with nitrogen gas three times $\left(\mathrm{AgSbF}_{6}\right.$ was taken inside the glove box). To the tube were then added $\mathrm{ClCH}_{2} \mathrm{CH}_{2} \mathrm{Cl}(3.0 \mathrm{~mL})$ via syringe, after that the reaction mixture was evacuated and purged with nitrogen gas three times. After that, the septum was taken out immediately and a screw cap was used to cover the tube under the nitrogen atmosphere and again the reaction mixture stirred at room temperature for 5 minutes. Then, the reaction mixture was allowed to stir at $100{ }^{\circ} \mathrm{C}$ for $16 \mathrm{~h}$ in oil bath. After cooling to ambient temperature, the reaction mixture was diluted with $\mathrm{CH}_{2} \mathrm{Cl}_{2}$, filtered through Celite and the filtrate was concentrated. The crude residue was purified through a 
silica gel column using hexanes and ethyl acetate as eluent to give pure product $\mathbf{6}$ in $72 \%$ yield under rhodium catalysis.

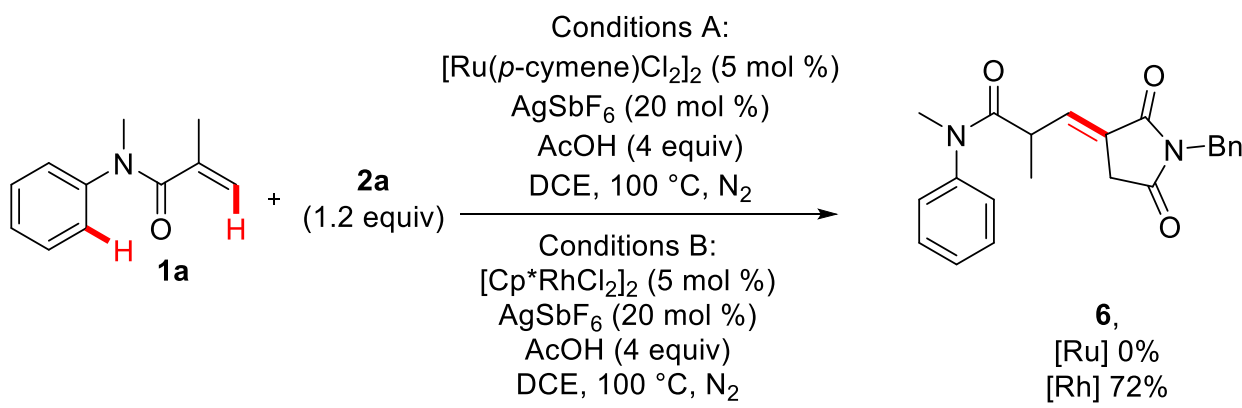

\section{Procedure for the Studies with Isotopically Labelled Compounds}

\section{A)H/D Exchange studies with $\mathrm{CD}_{3} \mathrm{OD}$ as Additive}

A $15 \mathrm{~mL}$ schlenk tube with septum containing $\left[\mathrm{RuCl}_{2}(\mathrm{p} \text {-cymene })\right]_{2}(5.0 \mathrm{~mol} \%), \mathrm{N}$ arylacrylamide1a (100mg, 1 equiv), $\mathrm{Cu}(\mathrm{OAc})_{2} \cdot \mathrm{H}_{2} \mathrm{O}$ (2.2equiv) and $\mathrm{AgSbF}_{6}(20 \mathrm{~mol} \%)$ was evacuated and purged with nitrogen gas three times ( $\mathrm{AgSbF}_{6}$ was taken inside the glove box). To the tube were then added $\mathrm{ClCH}_{2} \mathrm{CH}_{2} \mathrm{Cl}(3.0 \mathrm{~mL})$ via syringe, after that the reaction mixture was evacuated and purged with nitrogen gas three times. After that, the septum was taken out immediately and a screw cap was used to cover the tube under the nitrogen atmosphere and again the reaction mixture stirred at room temperature for 5 minutes. Then, the reaction mixture was allowed to stir at $100{ }^{\circ} \mathrm{C}$ for $16 \mathrm{~h}$ in oil bath. After cooling to ambient temperature, the reaction mixture was diluted with $\mathrm{CH}_{2} \mathrm{Cl}_{2}$, filtered through Celite and the filtrate was concentrated. The crude residue was directly analysed by $1 \mathrm{H}$ NMR.<smiles>C/C=C(/C)C(=O)N(C)c1ccccc1</smiles>

$1 \mathrm{a}$

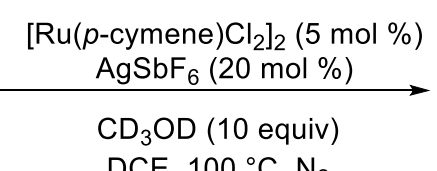

DCE, $100{ }^{\circ} \mathrm{C}, \mathrm{N}_{2}$<smiles>C/C(=C/OC(C)(C)C)C(=O)N(C)c1ccccc1</smiles>

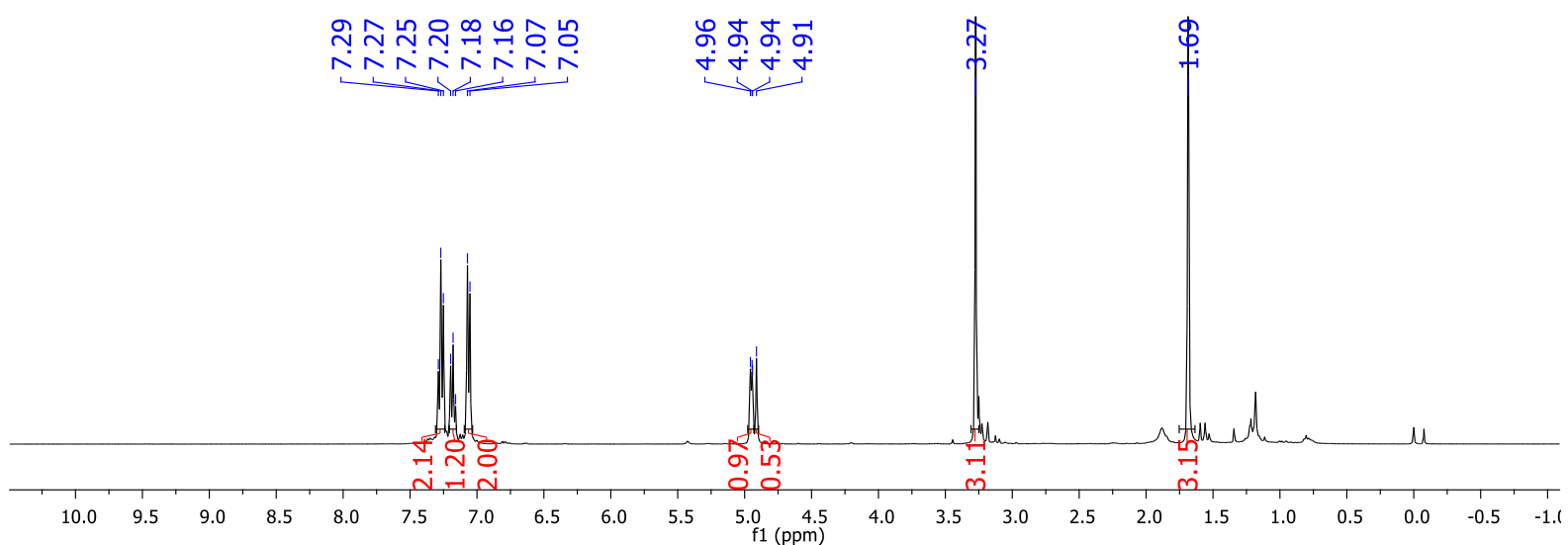




\section{B)H/D Exchange studies with $\mathrm{CD}_{3} \mathrm{OD}$ as Additive}

A $15 \mathrm{~mL}$ schlenk tube with septum containing $\left[\mathrm{RuCl}_{2}(\mathrm{p} \text {-cymene })\right]_{2}(5.0 \mathrm{~mol} \%), \mathrm{N}$ arylacrylamide1a (100mg, 1 equiv), maleimide $\mathbf{2 a}\left(1.2\right.$ equiv), $\mathrm{Cu}(\mathrm{OAc})_{2} \cdot \mathrm{H}_{2} \mathrm{O}$ (2.2equiv) and $\mathrm{AgSbF}_{6}(20 \mathrm{~mol} \%)$ was evacuated and purged with nitrogen gas three times $\left(\mathrm{AgSbF}_{6}\right.$ was taken inside the glove box). To the tube were then added $\mathrm{ClCH}_{2} \mathrm{CH}_{2} \mathrm{Cl}(2.0 \mathrm{~mL})$ and $\mathrm{CD}_{3} \mathrm{OD}$ $(1.0 \mathrm{~mL})$ via syringes, after that the reaction mixture was evacuated and purged with nitrogen gas three times. After that, the septum was taken out immediately and a screw cap was used to cover the tube under the nitrogen atmosphere and again the reaction mixture stirred at room temperature for 5 minutes. Then, the reaction mixture was allowed to stir at $100{ }^{\circ} \mathrm{C}$ for $16 \mathrm{~h}$ in oil bath. After cooling to ambient temperature, the reaction mixture was diluted with $\mathrm{CH}_{2} \mathrm{Cl}_{2}$, filtered through Celite and the filtrate was concentrated. The crude residue was purified through a silica gel column using hexanes and ethyl acetate as eluent to give pure product 3aa in $46 \%$ yield with $55 \%$ deuterium incorporation at the $\alpha$-position to the amide group in maleimide unit.<smiles>C/C=C(/C)C(=O)N(C)c1ccccc1</smiles>

$1 \mathrm{a}$

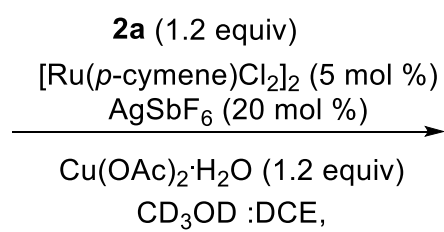

$(2: 2)$

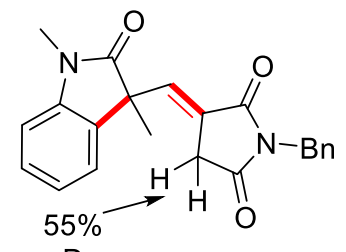

3aa, $46 \%$ $100^{\circ} \mathrm{C}, \mathrm{N}_{2}$

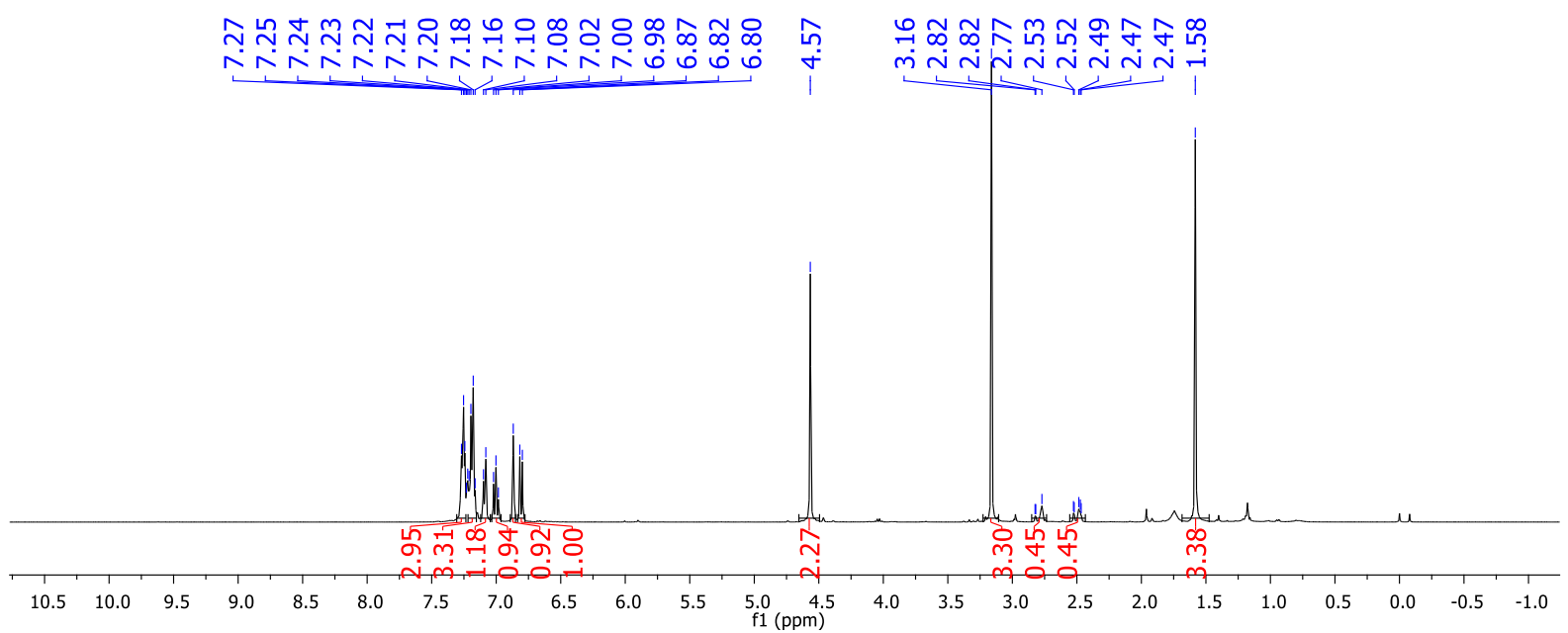




\section{B)H/D Exchange studies with [D]-1a and 2a}

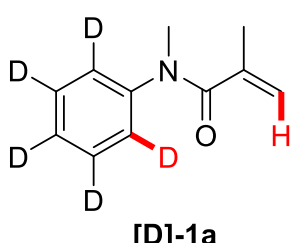

[D]-1a

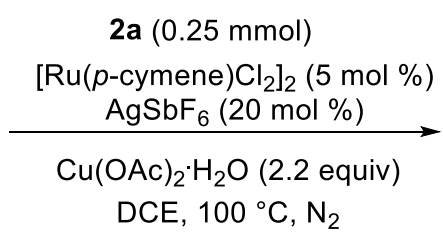

DCE, $100^{\circ} \mathrm{C}, \mathrm{N}_{2}$<smiles>[2H]c1c([2H])c([2H])c2c(c1[2H])N(C)C(=O)C2(C)/C=C1\CC(=O)N(Cc2ccccc2)C1=O</smiles>

[D]-3aa, $59 \%$

A $15 \mathrm{~mL}$ schlenk tube with septum containing $\left[\mathrm{RuCl}_{2}(\mathrm{p} \text {-cymene })\right]_{2}(5.0 \mathrm{~mol} \%), \mathrm{N}$ arylacrylamide[D]-1a (100mg, 1 equiv), maleimide $\mathbf{2 a}\left(1.2\right.$ equiv), $\mathrm{Cu}(\mathrm{OAc})_{2} \cdot \mathrm{H}_{2} \mathrm{O}$ (2.2equiv) and $\mathrm{AgSbF}_{6}(20 \mathrm{~mol} \%)$ was evacuated and purged with nitrogen gas three times $\left(\mathrm{AgSbF}_{6}\right.$ was taken inside the glove box). To the tube were then added $\mathrm{ClCH}_{2} \mathrm{CH}_{2} \mathrm{Cl}(3.0 \mathrm{~mL})$ via syringe, after that the reaction mixture was evacuated and purged with nitrogen gas three times. After that, the septum was taken out immediately and a screw cap was used to cover the tube under the nitrogen atmosphere and again the reaction mixture stirred at room temperature for 5 minutes. Then, the reaction mixture was allowed to stir at $100{ }^{\circ} \mathrm{C}$ for $16 \mathrm{~h}$ in oil bath. After cooling to ambient temperature, the reaction mixture was diluted with $\mathrm{CH}_{2} \mathrm{Cl}_{2}$, filtered through Celite and the filtrate was concentrated. The crude residue was purified through a silica gel column using hexanes and ethyl acetate as eluent to give pure product [D]-3aa in 59\% yield without any H/D exchange at the ortho position.

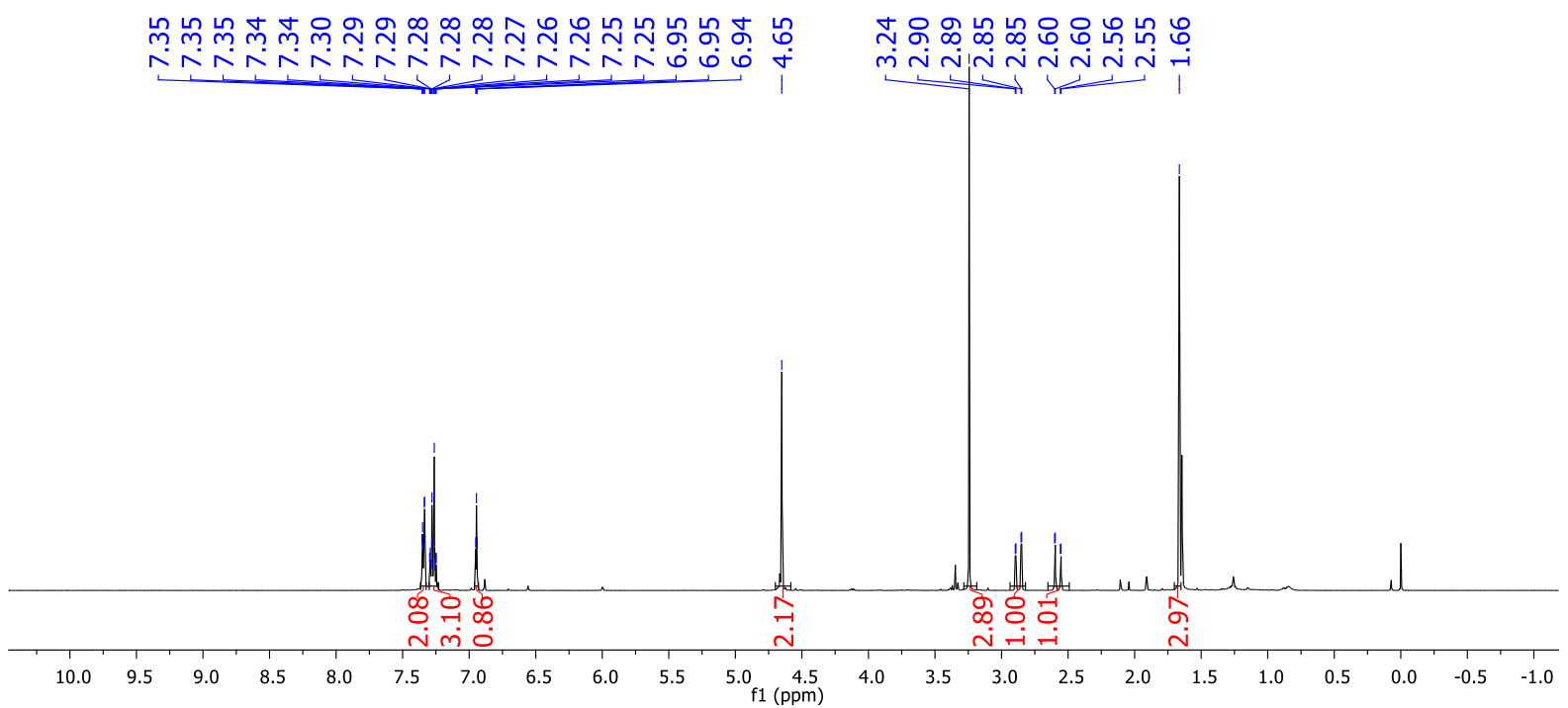

\section{Studies on the Kinetic Isotope Effect.}

\section{C) By competition reaction}

A $15 \mathrm{~mL}$ schlenk tube with septum containing $\left[\mathrm{RuCl}_{2}(\mathrm{p} \text {-cymene })\right]_{2}(5.0 \mathrm{~mol} \%), \mathrm{N}$ arylacrylamide1a $(0.25 \mathrm{mmol}, 1$ equiv), amide[D]-1a(0.25 mmol, 1 equiv), maleimide 2a(0.025 mmol, 1equiv), $\mathrm{Cu}(\mathrm{OAc})_{2} \cdot \mathrm{H}_{2} \mathrm{O}$ (2.2 equiv) and $\mathrm{AgSbF}_{6}(20 \mathrm{~mol} \%)$ was evacuated 
and purged with nitrogen gas three times $\left(\mathrm{AgSbF}_{6}\right.$ was taken inside the glove box). To the tube were then added $\mathrm{ClCH}_{2} \mathrm{CH}_{2} \mathrm{Cl}(3.0 \mathrm{~mL})$ via syringe, after that the reaction mixture was evacuated and purged with nitrogen gas three times. After that, the septum was taken out immediately and a screw cap was used to cover the tube under the nitrogen atmosphere and again the reaction mixture stirred at room temperature for 5 minutes. Then, the reaction mixture was allowed to stir at $100{ }^{\circ} \mathrm{C}$ for $16 \mathrm{~h}$ in oil bath. After cooling to ambient temperature, the reaction mixture was diluted with $\mathrm{CH}_{2} \mathrm{Cl}_{2}$, filtered through Celite and the filtrate was concentrated. The crude residue was purified through a silica gel column using hexanes and ethyl acetate as eluent to give combined products 3aaand [D]-3aain56\% yield.<smiles>C/C=C(/C)C(=O)N(C)c1ccc(Br)cc1</smiles>

$1 \mathrm{a} /[\mathrm{D}]-1 \mathrm{a}$ $0.25 \mathrm{mmol}$ each

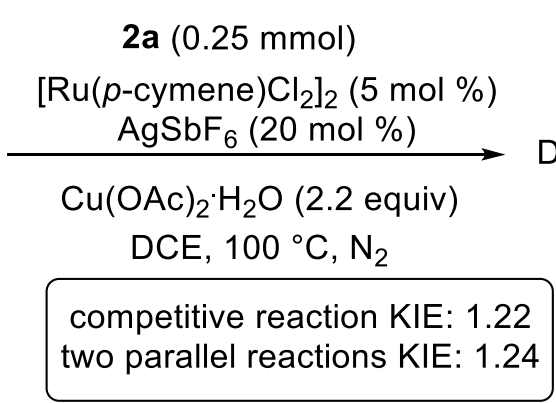

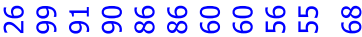

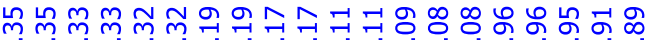

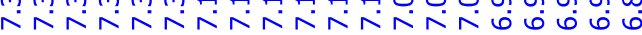<smiles>CN1C(=O)C(C)(/C=C2\CC(=O)N(Cc3ccccc3)C2=O)c2ccc([OH2+])cc21</smiles>

3aa, $56 \%$

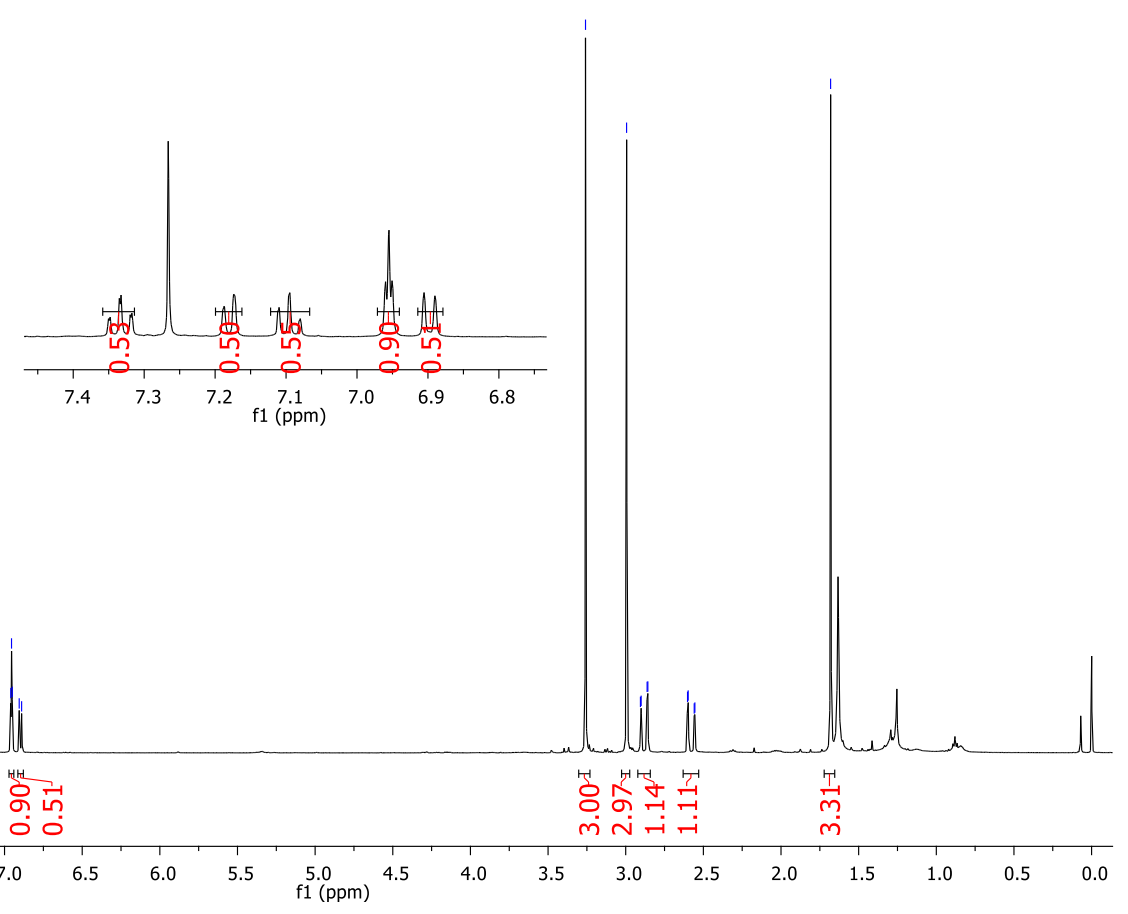




\section{B) By two parallel reactions}<smiles>C/C=C(/C)C(=O)N(C)c1ccccc1</smiles>

$1 \mathrm{a}$

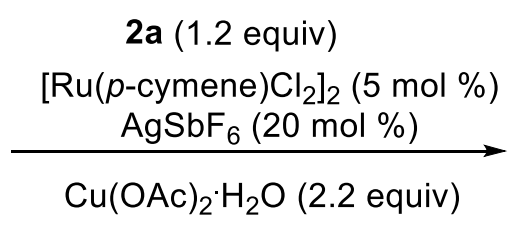

DCE, $100{ }^{\circ} \mathrm{C}, \mathrm{N}_{2}$<smiles>CN1C(=O)C(C)(/C=C2\CC(=O)N(Cc3ccccc3)C2=O)c2ccccc21</smiles>

A $15 \mathrm{~mL}$ schlenk tube with septum containing $\left[\mathrm{RuCl}_{2} \text { (p-cymene) }\right]_{2}(5.0 \mathrm{~mol} \%), \mathrm{N}$ arylacrylamide1a (100mg, 1 equiv), maleimide $\mathbf{2 a}\left(1.2\right.$ equiv), $\mathrm{Cu}(\mathrm{OAc})_{2} \cdot \mathrm{H}_{2} \mathrm{O}$ (2.2equiv) and $\mathrm{AgSbF}_{6}(20 \mathrm{~mol} \%)$ was evacuated and purged with nitrogen gas three times $\left(\mathrm{AgSbF}_{6}\right.$ was taken inside the glove box $)$. To the tube were then added $\mathrm{ClCH}_{2} \mathrm{CH}_{2} \mathrm{Cl}(2.0 \mathrm{~mL})$ and $\mathrm{CD}_{3} \mathrm{OD}$ $(1.0 \mathrm{~mL})$ via syringes, after that the reaction mixture was evacuated and purged with nitrogen gas three times. After that, the septum was taken out immediately and a screw cap was used to cover the tube under the nitrogen atmosphere and again the reaction mixture stirred at room temperature for 5 minutes. Then, the reaction mixture was allowed to stir at $100{ }^{\circ} \mathrm{C}$ in oil bath. For $120 \mathrm{~min}$, an aliquot $(0.1 \mathrm{~mL})$ was removed by a syringe every $20 \mathrm{~min}$ and directly analyzed by $1 \mathrm{H}-\mathrm{NMR}$.<smiles>[2H]c1c([2H])c([2H])c(N(C)C(=O)/C(C)=C\C)c([2H])c1[2H]</smiles>

[D]-1a

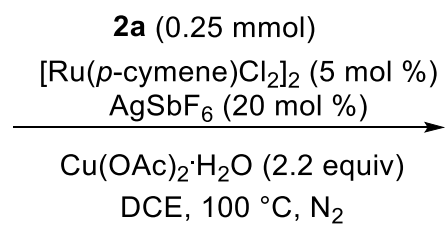<smiles>[2H]c1c([2H])c([2H])c2c(c1[2H])N(C)C(=O)C2(C)/C=C1\CC(=O)N(Cc2ccccc2)C1=O</smiles>

The procedure above was followed using [D]-1a (100 mg,). Data from independent kinetic isotope studies are collected in the figure below and KIE was found to be $k_{\mathrm{H}} / k_{\mathrm{D}} \approx 1.24$ 


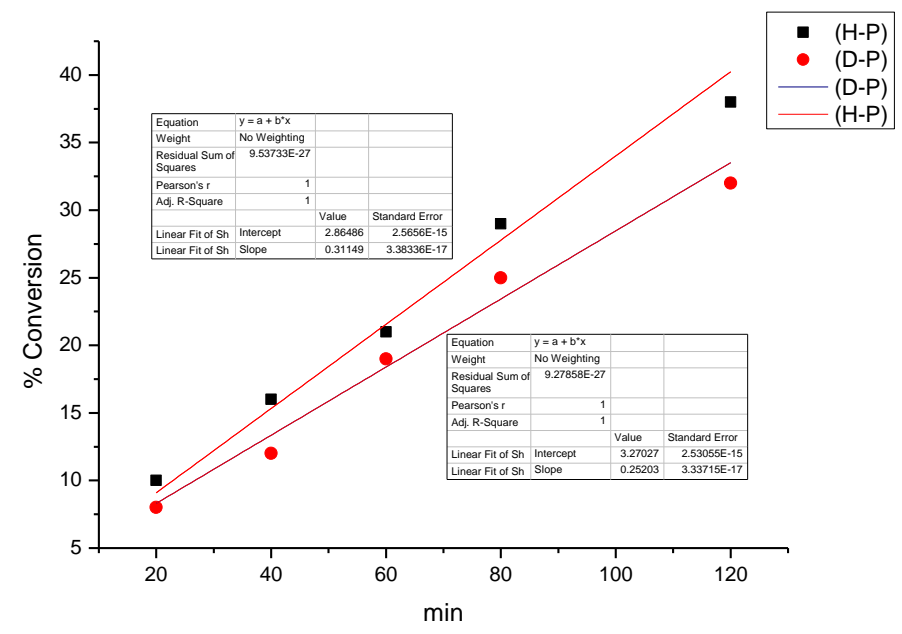

\section{Radical trapping experiments}

A $15 \mathrm{~mL}$ schlenk tube with septum containing $\left[\mathrm{RuCl}_{2} \text { (p-cymene) }\right]_{2}(5.0 \mathrm{~mol} \%), \mathrm{N}$ arylacrylamide1a (100mg, 1 equiv), maleimide $\mathbf{2 a}\left(1.2\right.$ equiv), $\mathrm{Cu}(\mathrm{OAc})_{2} \cdot \mathrm{H}_{2} \mathrm{O}$ (2.2equiv), $\mathrm{AgSbF}_{6}(20 \mathrm{~mol} \%)$ and TEMPO or BHT (2 equiv) was evacuated and purged with nitrogen gas three times $\left(\mathrm{AgSbF}_{6}\right.$ was taken inside the glove box). To the tube were then added $\mathrm{ClCH}_{2} \mathrm{CH}_{2} \mathrm{Cl}(2.0 \mathrm{~mL})$ and $\mathrm{CD}_{3} \mathrm{OD}(1.0 \mathrm{~mL})$ via syringes, after that the reaction mixture was evacuated and purged with nitrogen gas three times. After that, the septum was taken out immediately and a screw cap was used to cover the tube under the nitrogen atmosphere and again the reaction mixture stirred at room temperature for 5 minutes. Then, the reaction mixture was allowed to stir at $100{ }^{\circ} \mathrm{C}$ for $16 \mathrm{~h}$ in oil bath. In crude reaction mixture expected product 3aawas not observed.<smiles>C/C=C(/C)C(=O)N(C)c1ccccc1</smiles>

$1 \mathrm{a}$

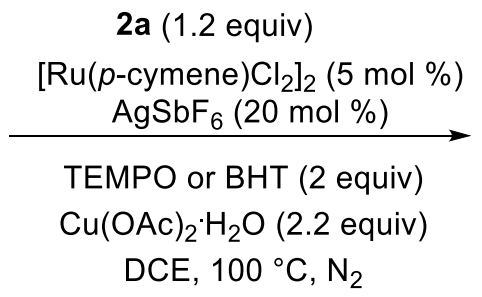<smiles>CN1C(=O)C(C)(/C=C2\CC(=O)N(Cc3ccccc3)C2=O)c2ccccc21</smiles>

3aa, $0 \%$ 


\section{Single crystal X-Ray Studies}

Suitable single crystals for X-ray diffraction studies were obtained from the compound synthesized in this study. Single crystals were grown in a NMR tube at room temperature using $\mathrm{CDCl}_{3}$ over a period of 3-4 weeks by slow evaporation of solvent.

X-ray data was collected with a Bruker AXS (Kappa Apex 2) CCD diffractometer equipped with graphite monochromatic Mo $(\mathrm{K} \alpha)(\lambda=0.7107 \mathrm{~A})$ radiation source. The data were collected with $100 \%$ completeness for $\Theta$ up to $25^{\circ}$. $\omega$ and $\phi$ scans were employed to collect the data. The frame width for $\omega$ for was fixed to $0.5^{\circ}$ for data collection. The crystal was solved by direct methods using Bruker SHELXS (Sheldrick, 1997). The Structure was refined using the Bruker SHELXTL (Version 6.12) software package. These data were deposited with Cambridge Crystallographic Data Center with the following numbers: CCDC 1919981 and CCDC 1919647.

ORTEP representation of compound 3ia displaying thermal elliposoid at 50\% probability.

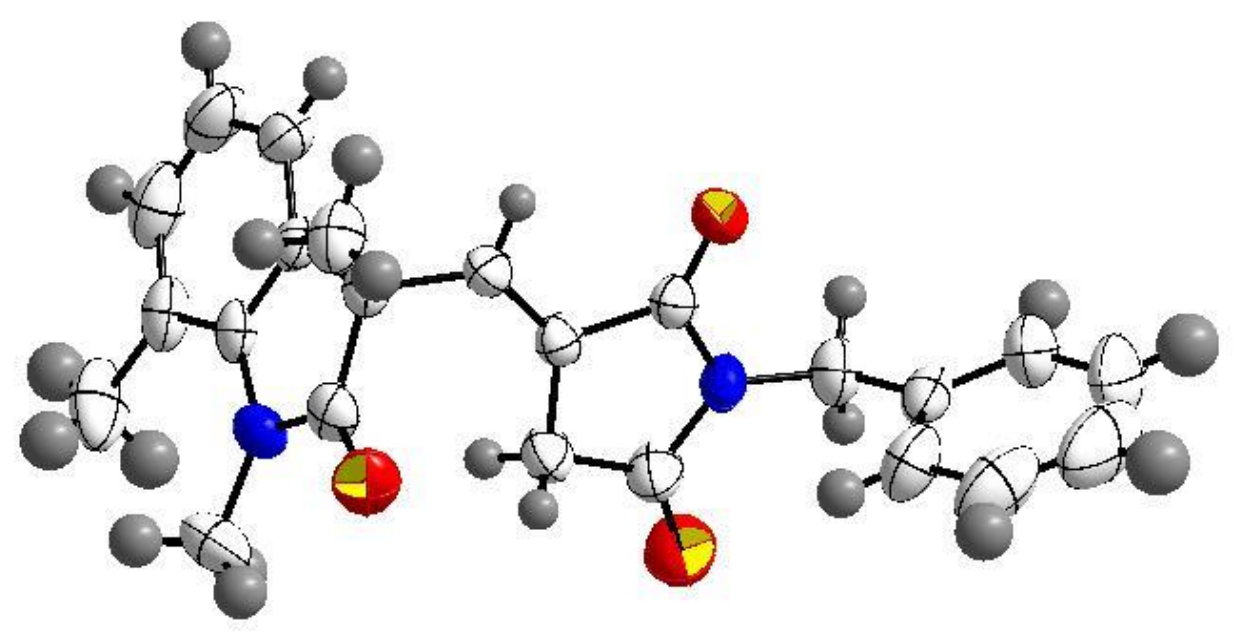

Table S1.Crystal data and structure refinement for compound 3ia.

Identification code

nrm-4272

Empirical formula

$\mathrm{C}_{23} \mathrm{H}_{22} \mathrm{~N}_{2} \mathrm{O}_{3}$

Formula weight

374.42 
Temperature

Wavelength

Crystal system

Space group

Unit cell dimensions

Volume

$\mathrm{Z}$

Density (calculated)

Absorption coefficient $\mathrm{F}(000)$

Theta range for data collection

Index ranges

Reflections collected

Independent reflections

Completeness to theta $=24.999^{\circ}$

Goodness-of-fit on $\mathrm{F}^{2}$

$\mathrm{R}$ indices (all data)

Extinction coefficient
$296 \mathrm{~K}$

$0.71073 \AA$

orthorhombic

$\mathrm{P}-21$

$\mathrm{a}=8.9275(5) \AA \quad \mathrm{a}=90^{\circ}$.

$\mathrm{b}=10.3757(7) \AA \quad \mathrm{b}=90^{\circ}$

$\mathrm{c}=21.3167(13) \AA \quad \mathrm{g}=90^{\circ}$

$1974.5(2) \AA^{3}$

4

$1.260 \mathrm{~g} / \mathrm{cm}^{-3}$

$0.084 \mathrm{~mm}^{-1}$

792

1.692 to $24.999^{\circ}$.

$-15<=\mathrm{h}<=15,-5<=\mathrm{k}<=7,-28<=\mathrm{l}<=24$

3066

$2248[\mathrm{R}(\mathrm{int})=0.0881]$

$99.9 \%$

1.020

$\mathrm{R} 1=0.0405, \mathrm{wR} 2=0.1084$

$\mathrm{n} / \mathrm{a}$ 
ORTEP representation of compound 3af displaying thermal elliposoid at 50\% probability.

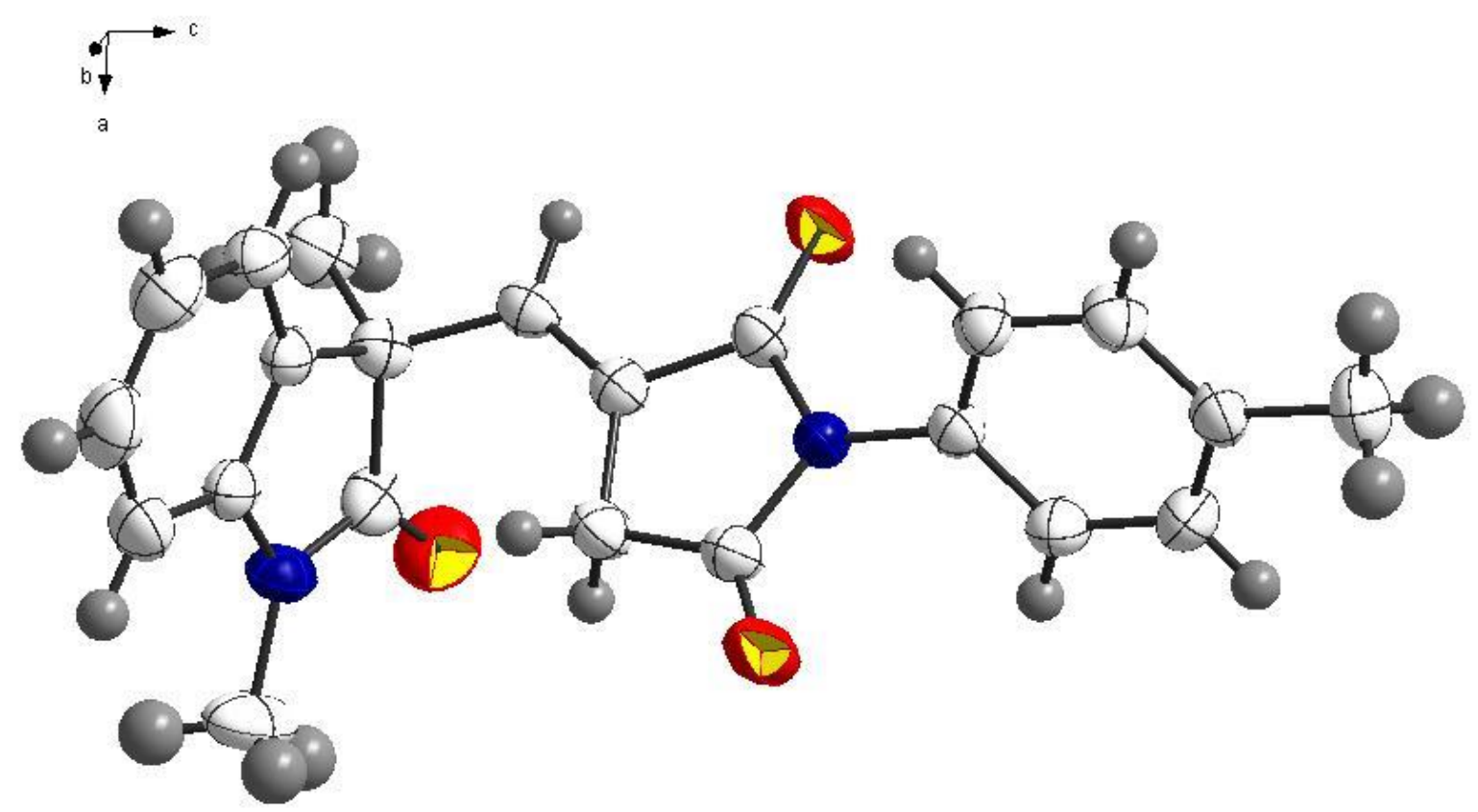

Table S2.Crystal data and structure refinement for compound 3af.

Identification code

Empirical formula

Formula weight

Temperature

Wavelength

Crystal system

Space group

Unit cell dimensions

Volume

Z

Density (calculated)

Absorption coefficient $\mathrm{F}(000)$ nrm-4281

$\mathrm{C}_{22} \mathrm{H}_{20} \mathrm{~N}_{2} \mathrm{O}_{3}$

360.40

$296 \mathrm{~K}$

$0.71073 \AA$

monoclinic

$\mathrm{P}-21 / \mathrm{n}$

$\mathrm{a}=12.6714(4) \AA \quad \mathrm{a}=90^{\circ}$.

$\mathrm{b}=6.1189(2) \AA$

$\mathrm{b}=98.6402(13)^{\circ}$

$\mathrm{c}=24.3445(8) \AA$

$\mathrm{g}=90^{\circ}$

1866.13(10) $\AA^{3}$

4

$1.283 \mathrm{~g} / \mathrm{cm}^{-3}$

$0.086 \mathrm{~mm}^{-1}$

760 
Theta range for data collection

Index ranges

Reflections collected

Independent reflections

Completeness to theta $=24.999^{\circ}$

Goodness-of-fit on $\mathrm{F}^{2}$

$\mathrm{R}$ indices (all data)

Extinction coefficient
1.692 to $24.999^{\circ}$.

$-15<=\mathrm{h}<=15,-5<=\mathrm{k}<=7,-28<=1<=24$

5947

$3289[\mathrm{R}(\mathrm{int})=0.0881]$

$99.9 \%$

1.033

$\mathrm{R} 1=0.0405, \mathrm{wR} 2=0.1084$

$\mathrm{n} / \mathrm{a}$ 


\section{Copies of ${ }^{1} \mathrm{H}$ and ${ }^{13} \mathrm{C}\{1 \mathrm{H}\}$ NMR Spectra}

Copies of $\mathrm{H}^{1}, \mathrm{C}^{13}$ and DEPT 135 NMR spectra of compound 3aa

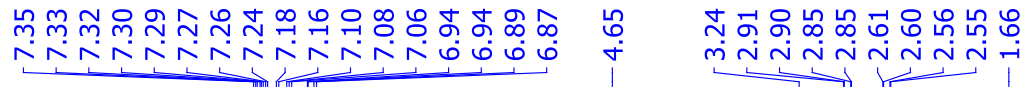
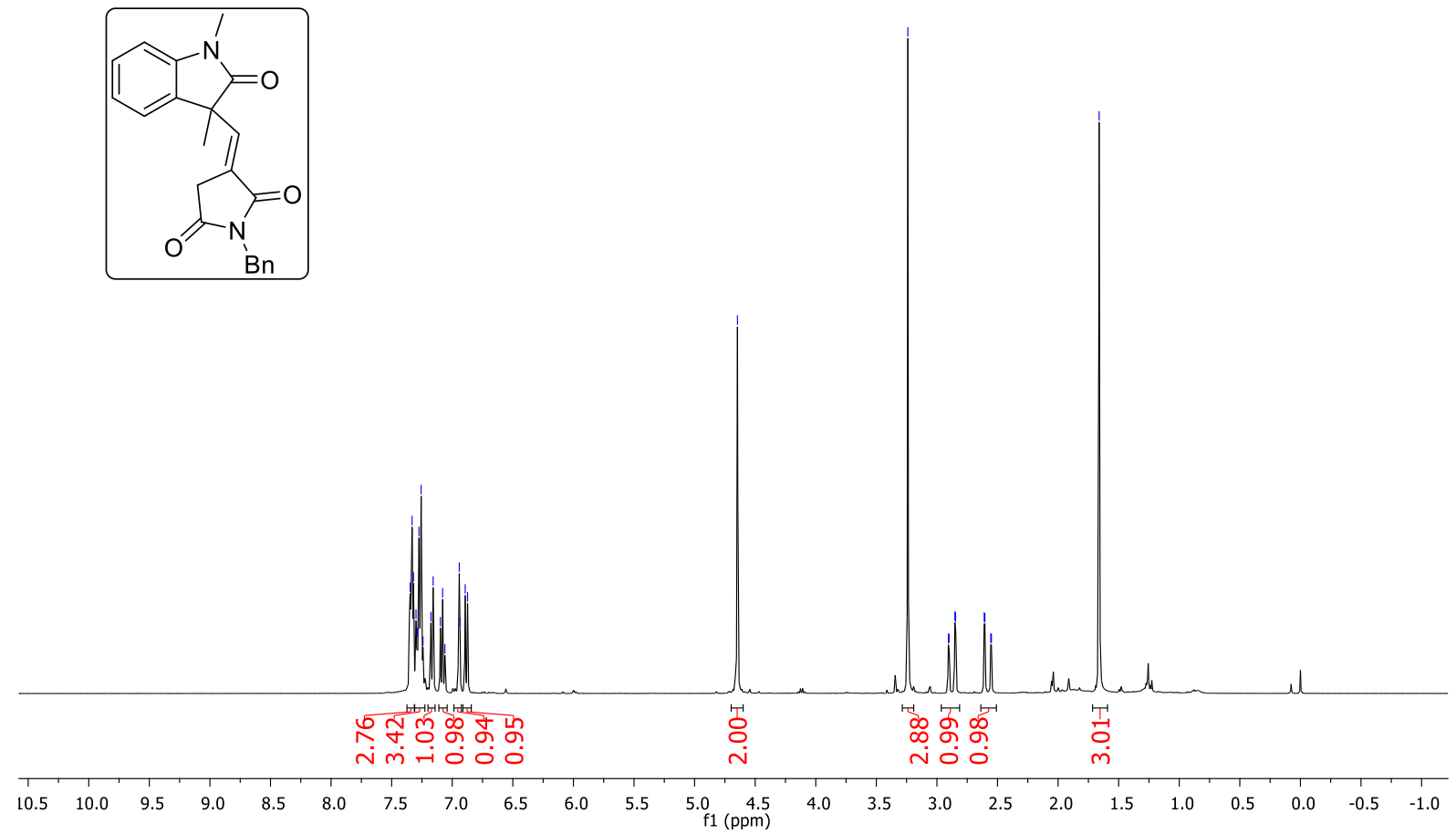

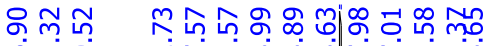

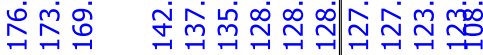

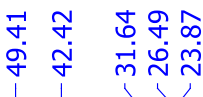

$\begin{array}{lllllllllllllllllllllll}210 & 200 & 190 & 180 & 170 & 160 & 150 & 140 & 130 & 120 & 110 & \begin{array}{c}100 \\ \mathrm{f} 1(\mathrm{pmm})\end{array} & 90 & 80 & 70 & 60 & 50 & 40 & 30 & 20 & 10 & 0 & -10\end{array}$ 


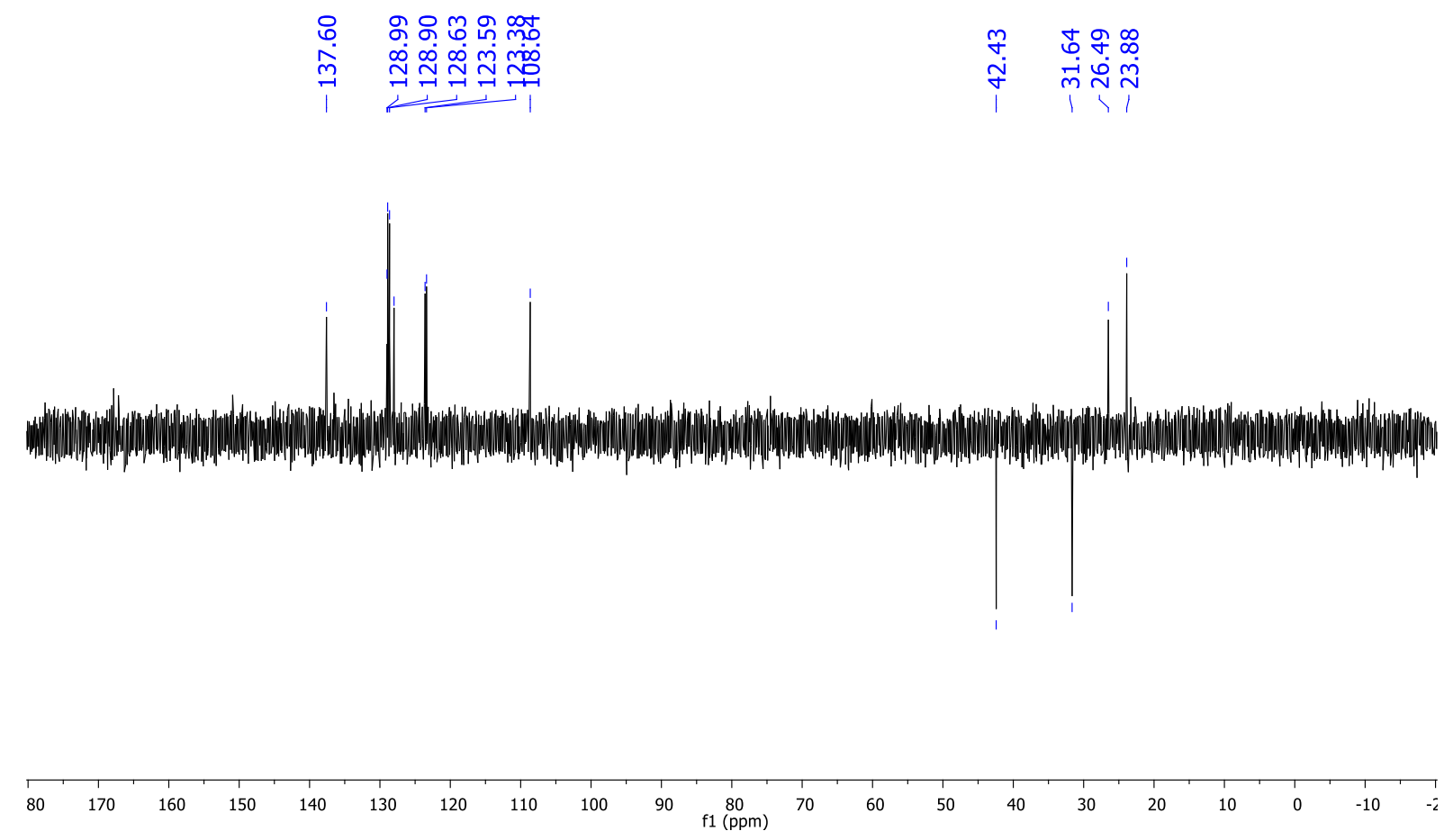


Copies of $\mathrm{H}^{1}, \mathrm{C}^{13}$ and DEPT 135 NMR spectra of compound $\mathbf{3 b a}$

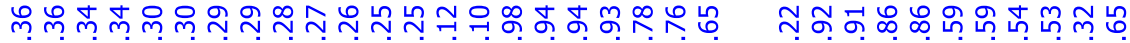

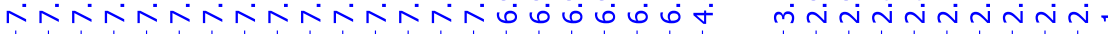

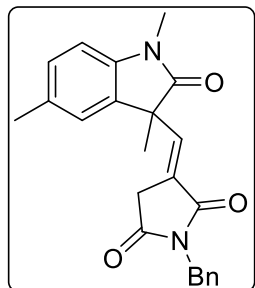

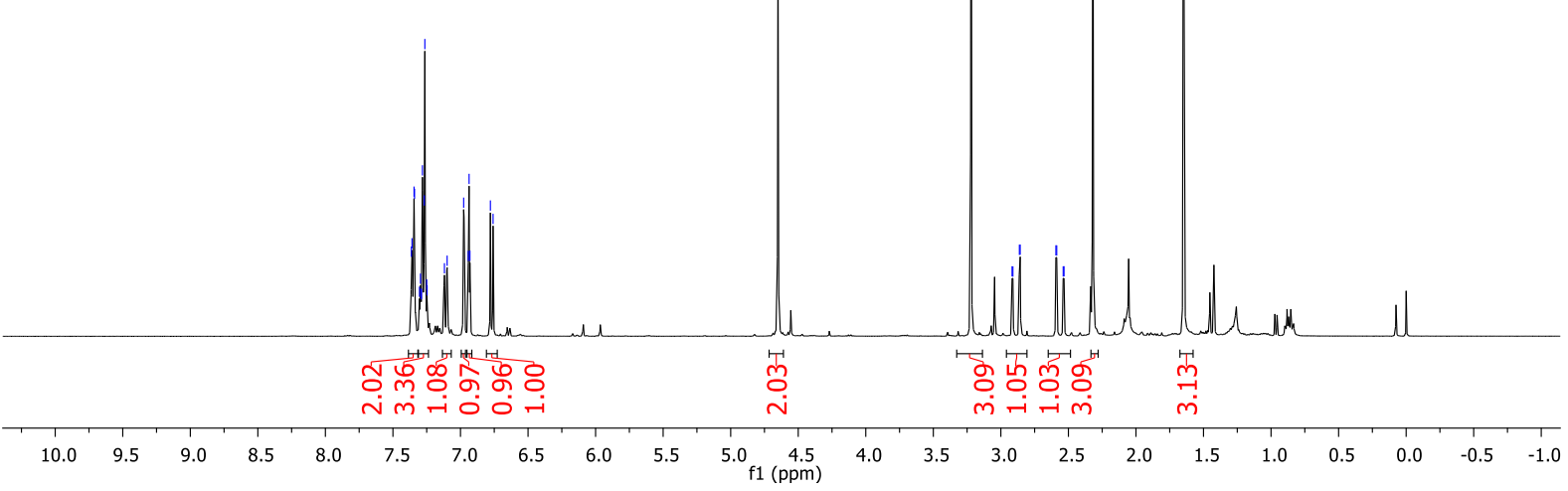

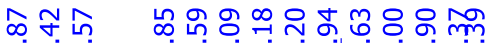

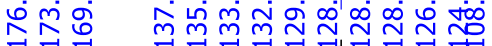

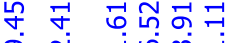

守

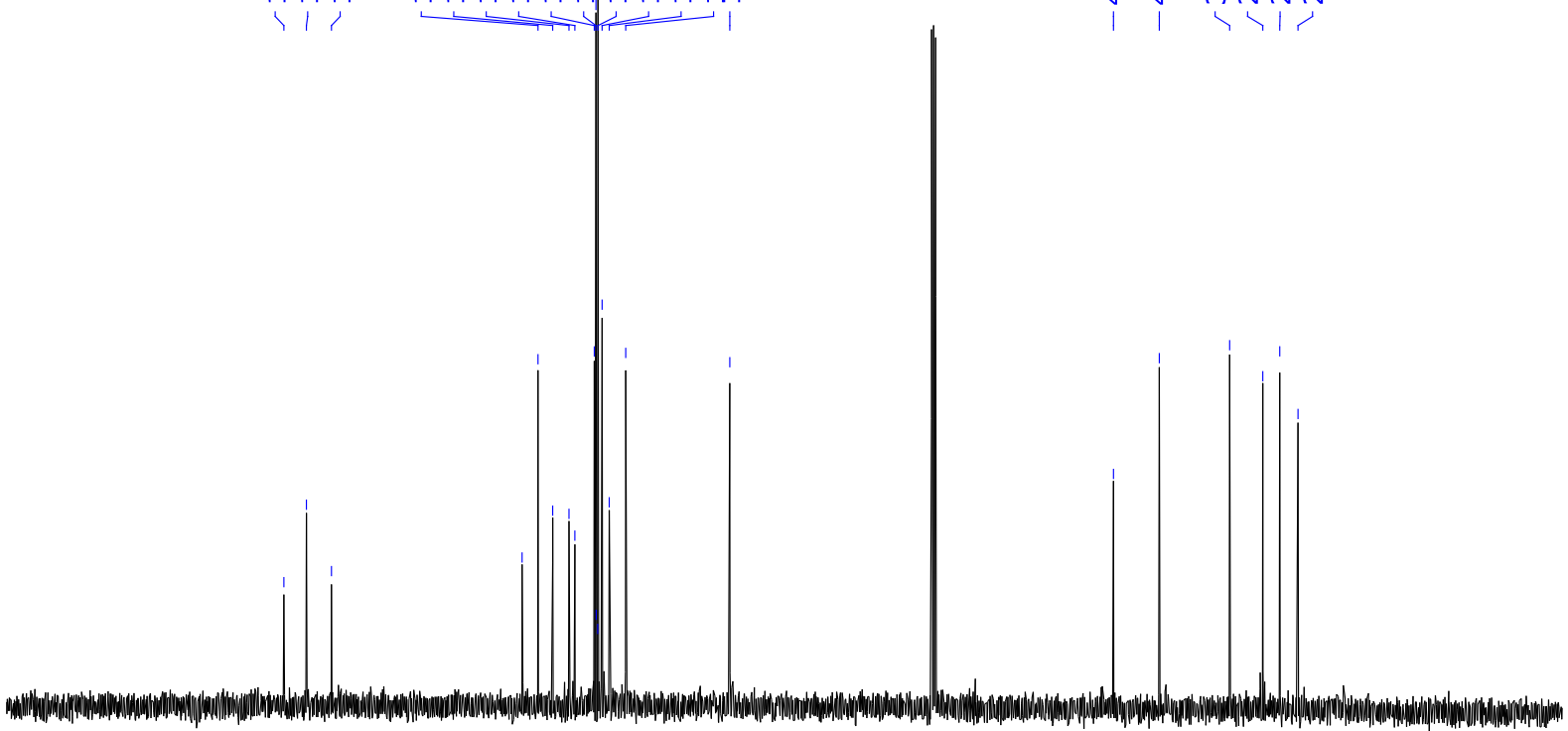

$\begin{array}{lllllllllllllllllllllllllll}210 & 200 & 190 & 180 & 170 & 160 & 150 & 140 & 130 & 120 & 110 & 100 & 90 & 80 & 70 & 60 & 50 & 40 & 30 & 20 & 10 & 0 & -10 & \end{array}$ 


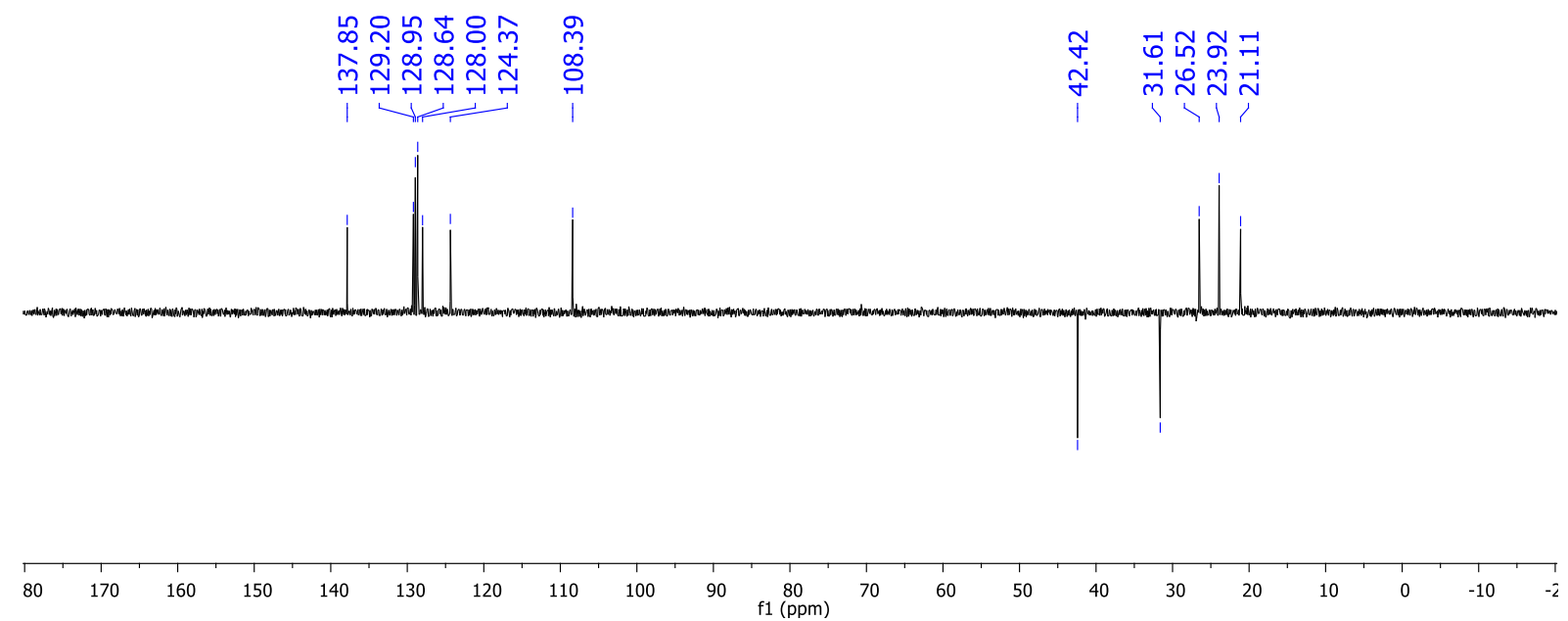


Copies of $\mathrm{H}^{1}, \mathrm{C}^{13}$ and DEPT 135 NMR spectra of compound 3ca

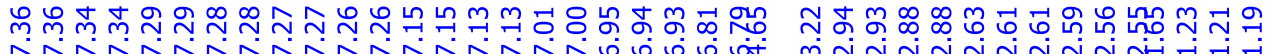

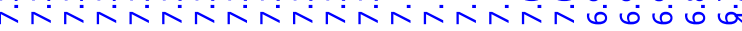

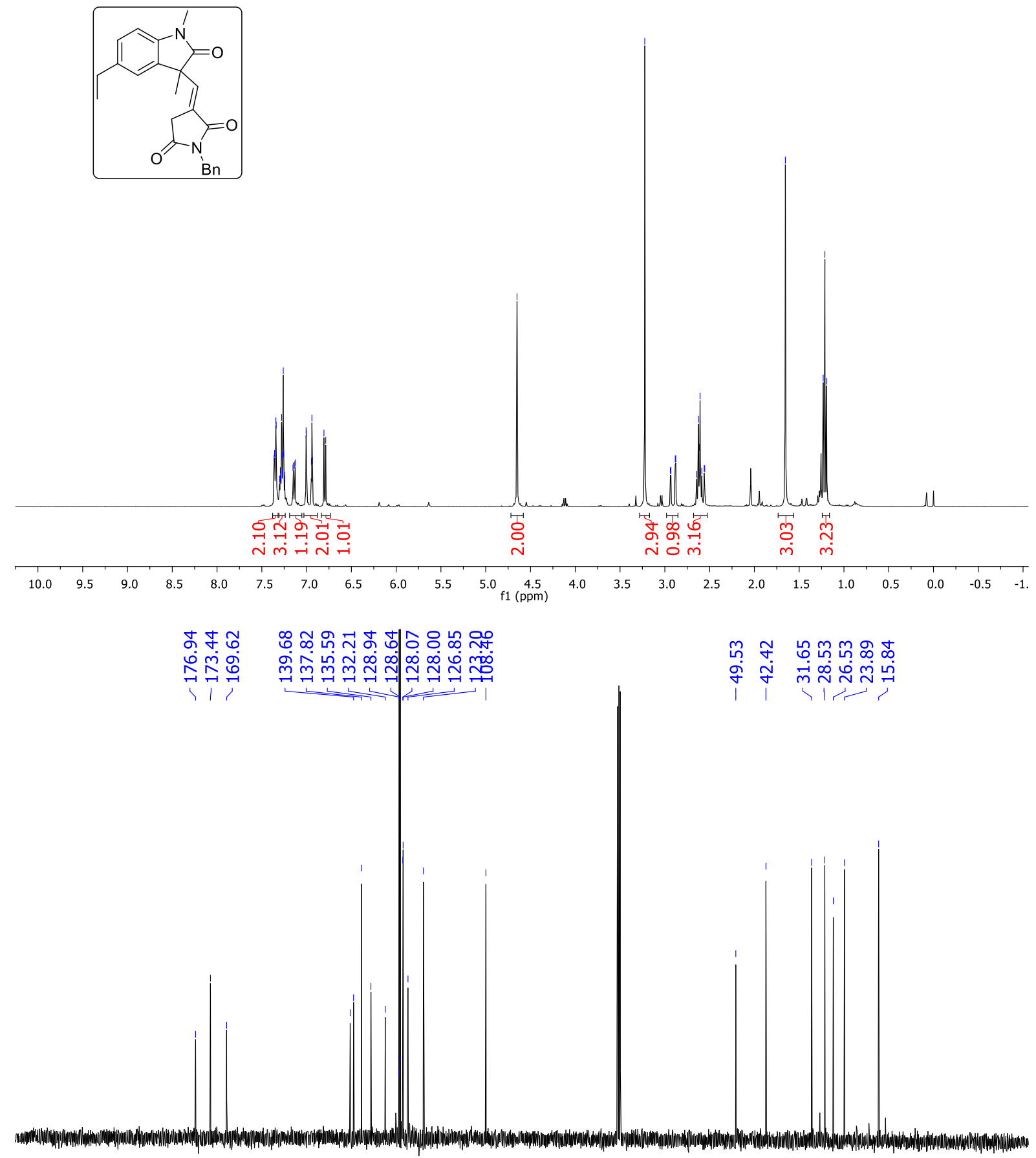
ming

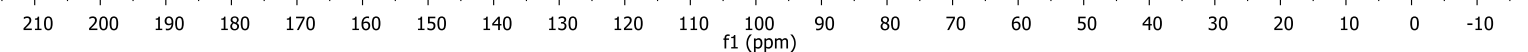




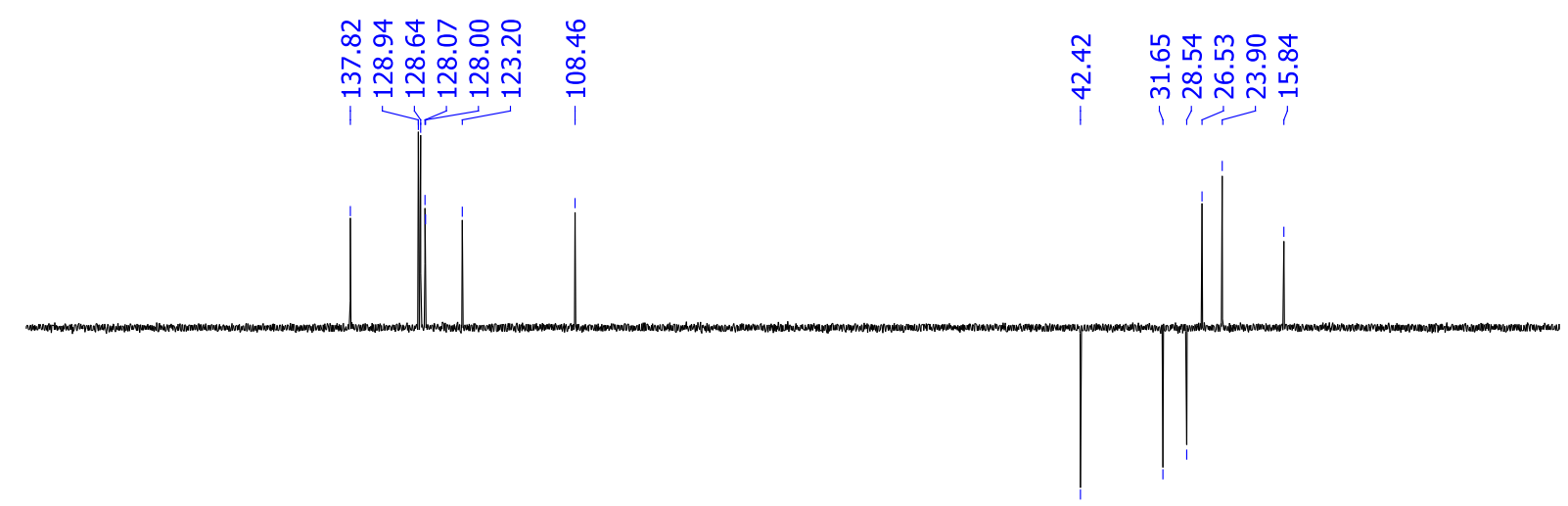

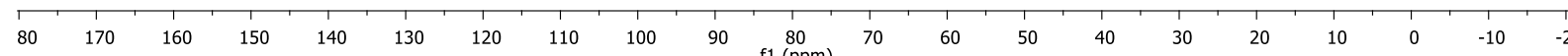


Copies of $\mathrm{H}^{1}, \mathrm{C}^{13}$ and DEPT 135 NMR spectra of compound 3da

ల్లిల్లి

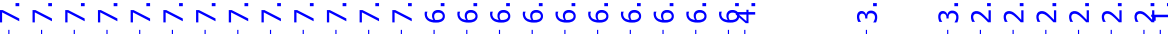
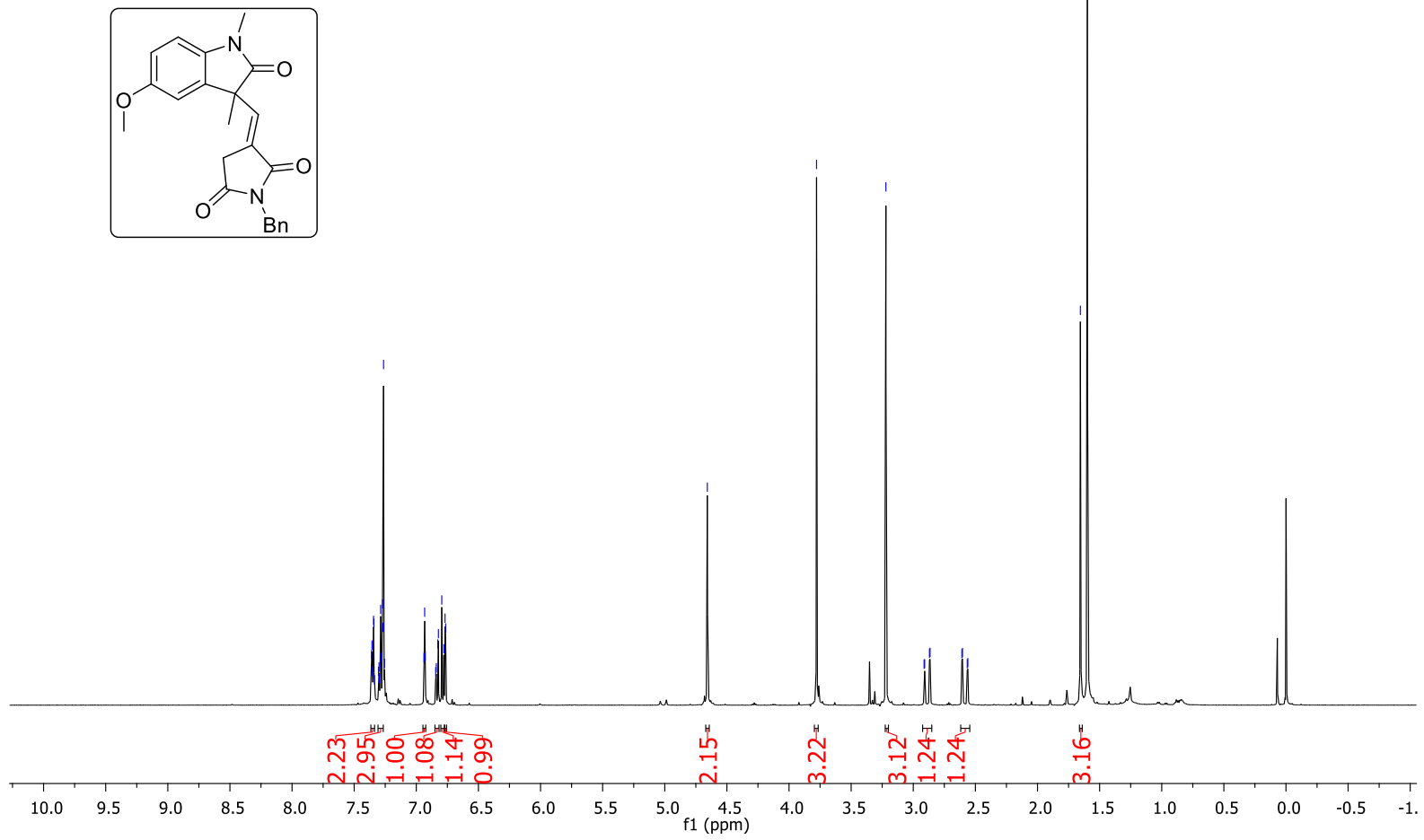

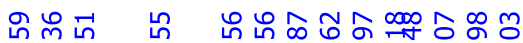

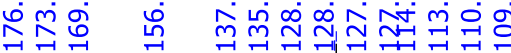

1, ।

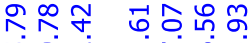

นึ่

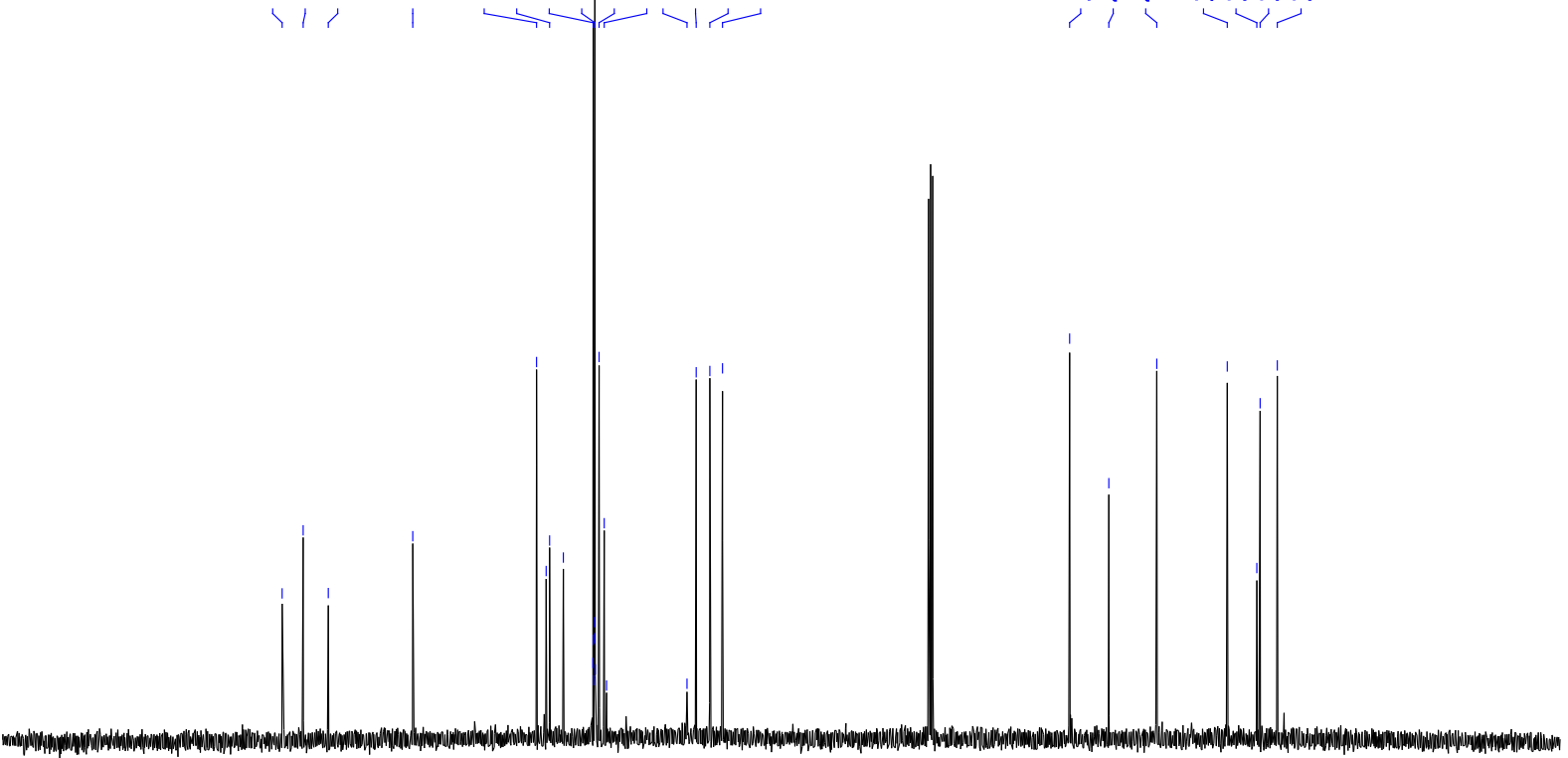

$\begin{array}{lllllllllllllllllllllll}210 & 200 & 190 & 180 & 170 & 160 & 150 & 140 & 130 & 120 & 110 & \underset{\mathrm{f} 1}{100}(\mathrm{ppm}) & 90 & 80 & 70 & 60 & 50 & 40 & 30 & 20 & 10 & 0 & -10\end{array}$ 


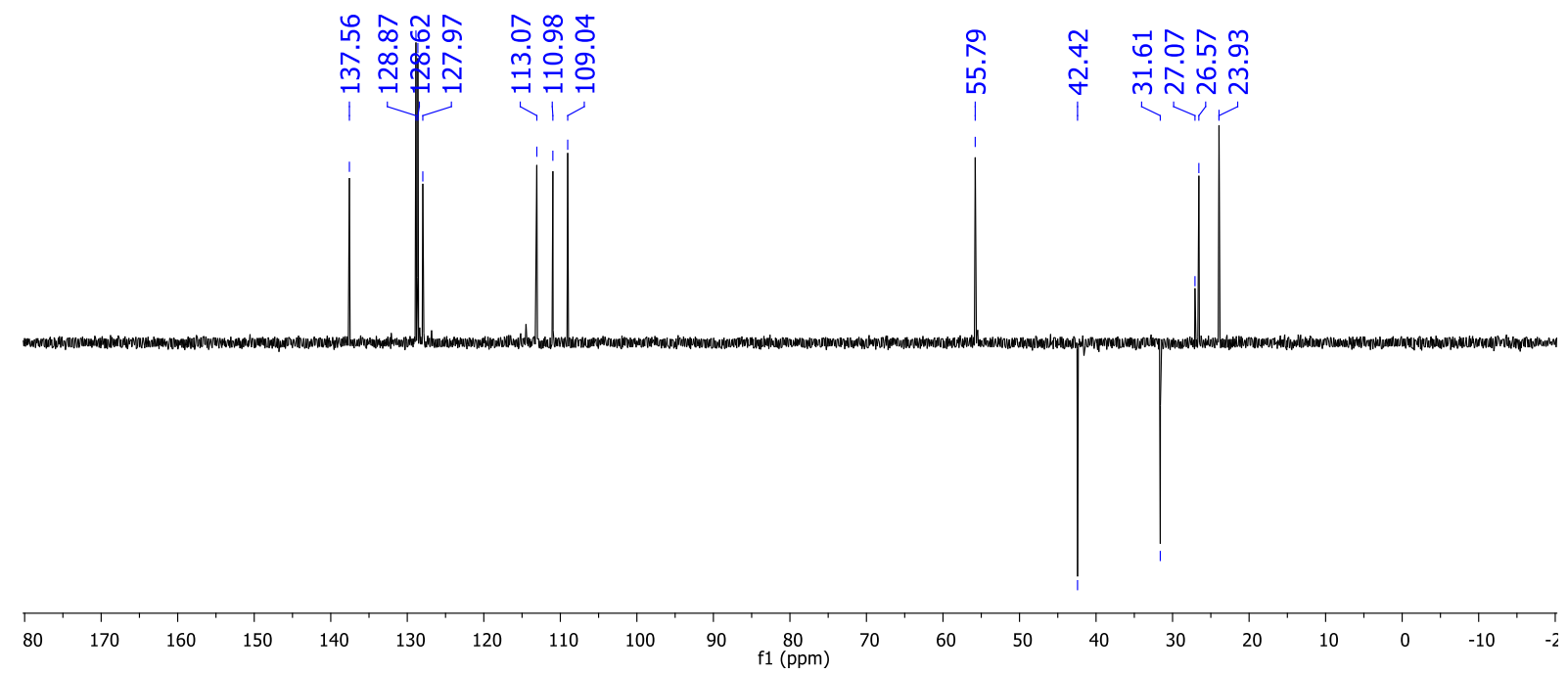


Copies of $\mathrm{H}^{1}, \mathrm{C}^{13}$ and DEPT 135 NMR spectra of compound 3ea

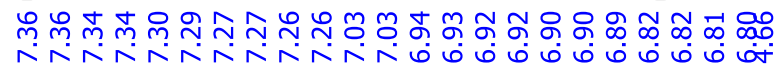

(n)

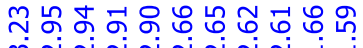

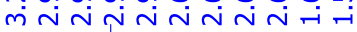

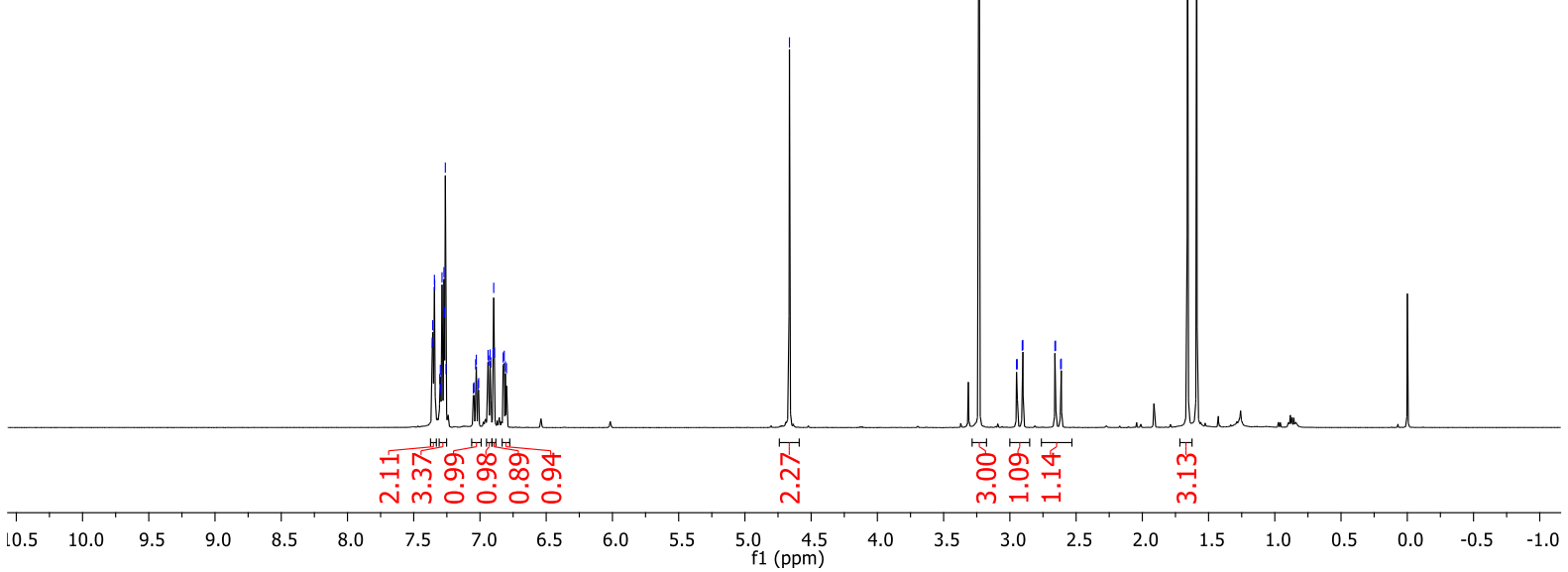

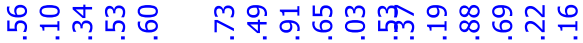

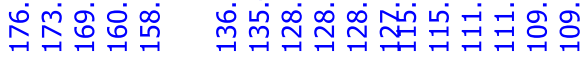

似

뉴 유용ㅇ

वे

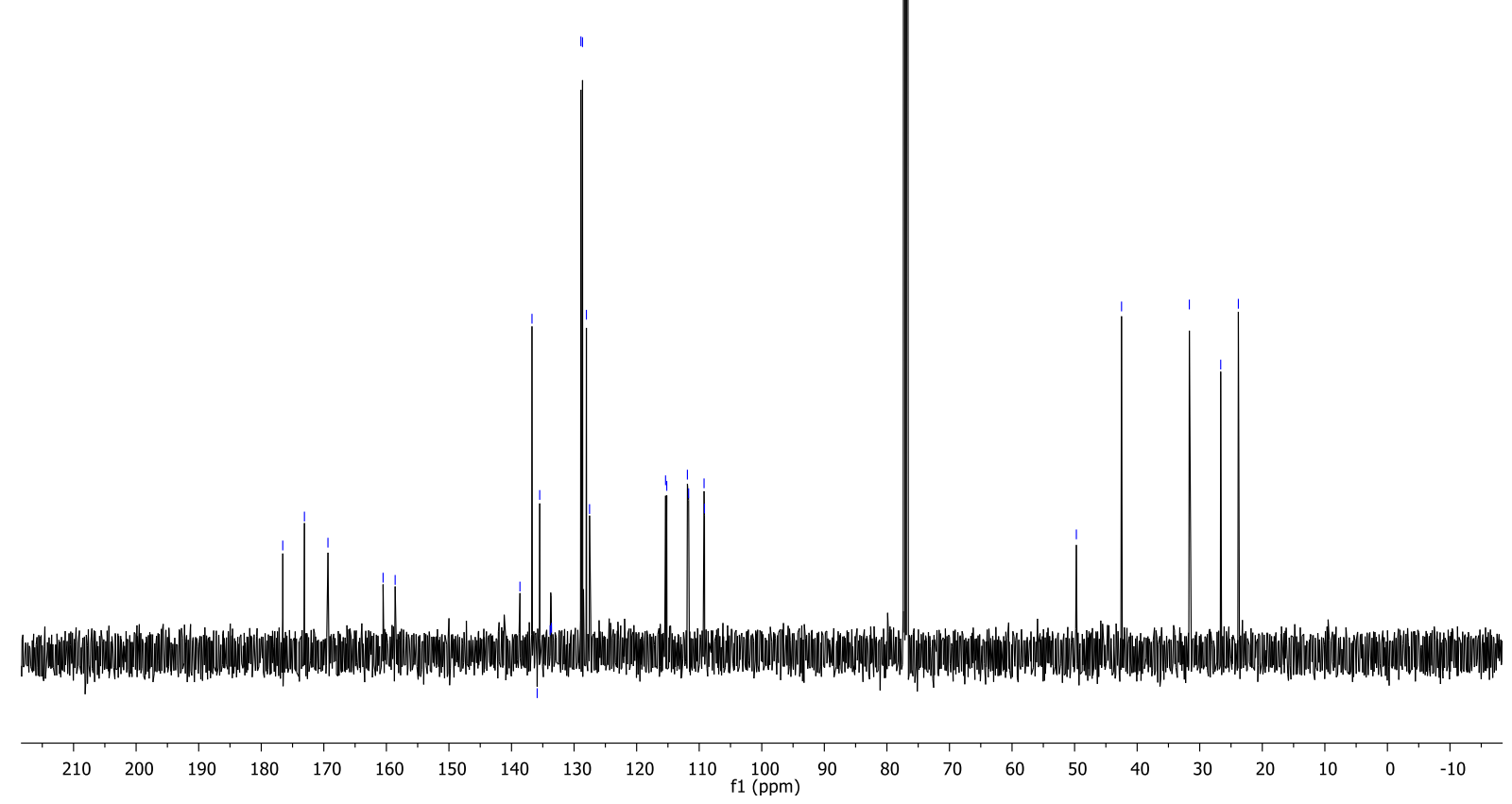




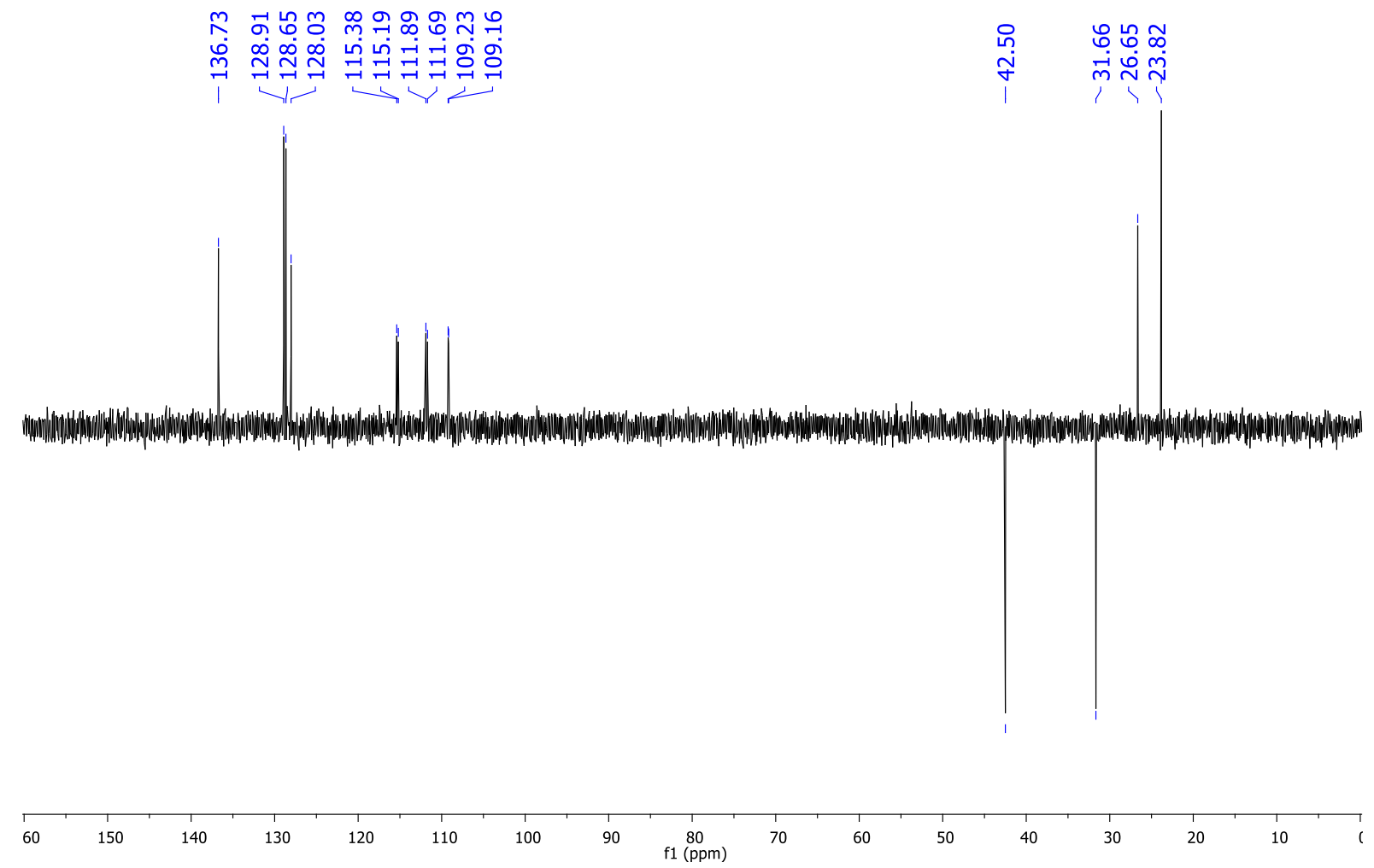


Copies of $\mathrm{H}^{1}, \mathrm{C}^{13}$ and DEPT 135 NMR spectra of compound $\mathbf{3 f a}$
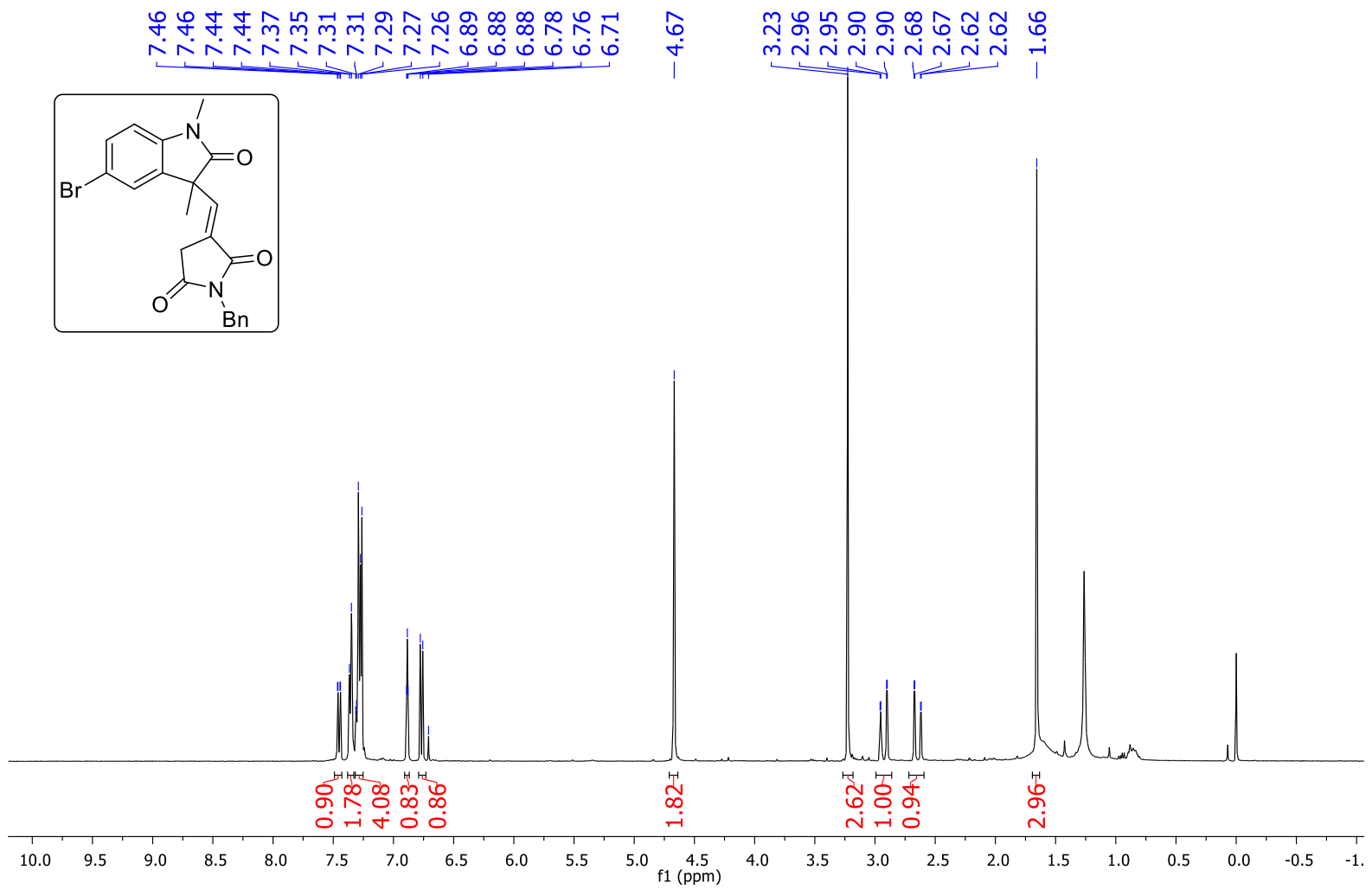

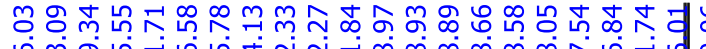

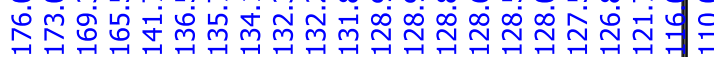

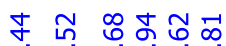
ชั

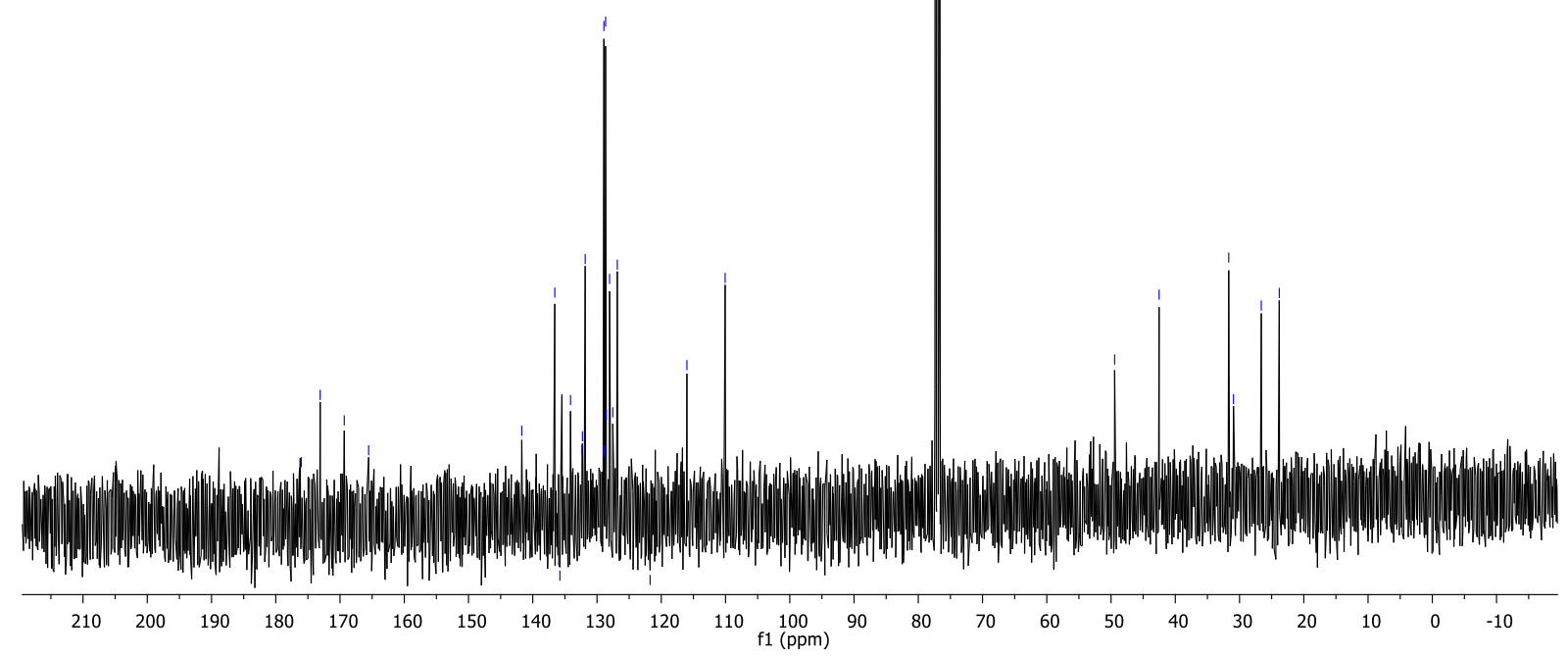




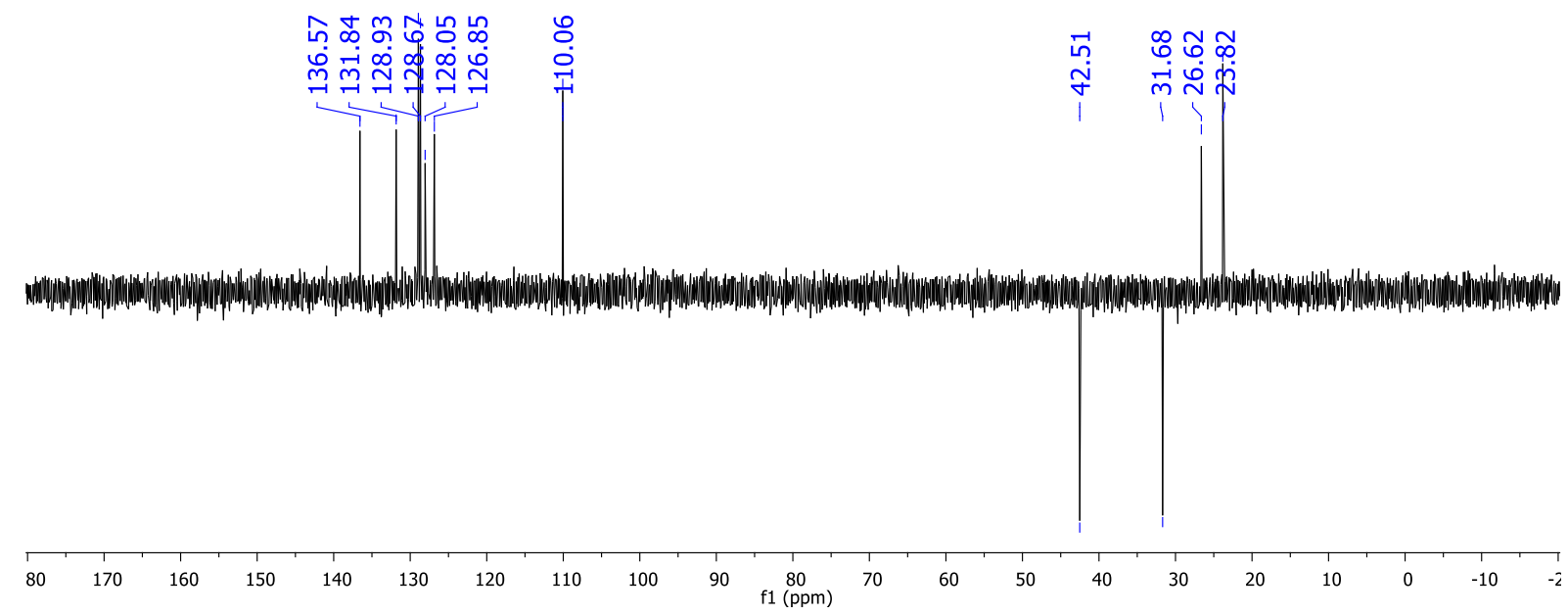


Copies of $\mathrm{H}^{1}, \mathrm{C}^{13}$ and DEPT 135 NMR spectra of compound 3ga

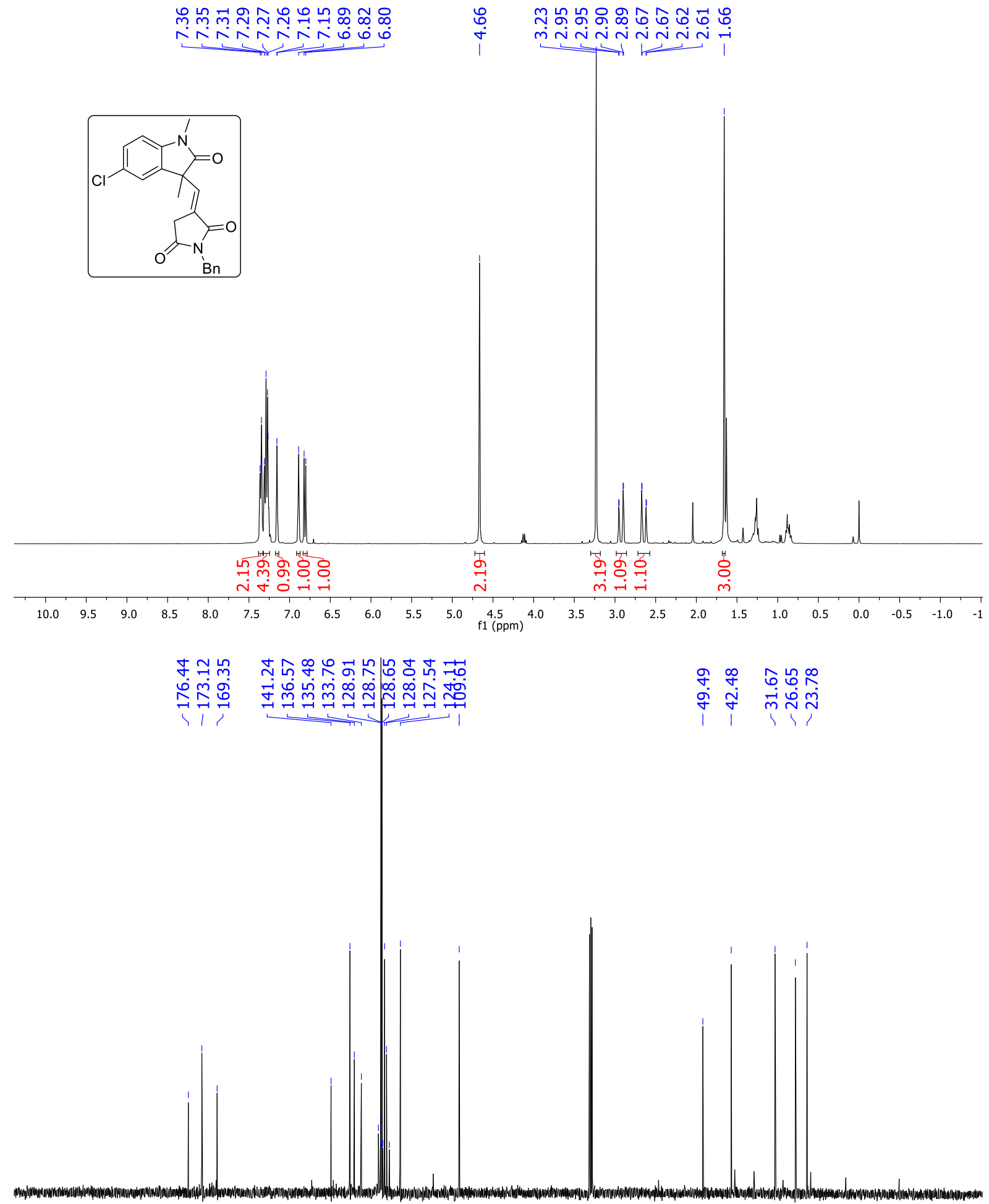

$\begin{array}{lllllllllllllllllllllll}210 & 200 & 190 & 180 & 170 & 160 & 150 & 140 & 130 & 120 & 110 & \underset{\mathrm{f} 1}{100}(\mathrm{ppm}) \\ 90 & 80 & 70 & 60 & 50 & 40 & 30 & 20 & 10 & 0 & -10\end{array}$ 


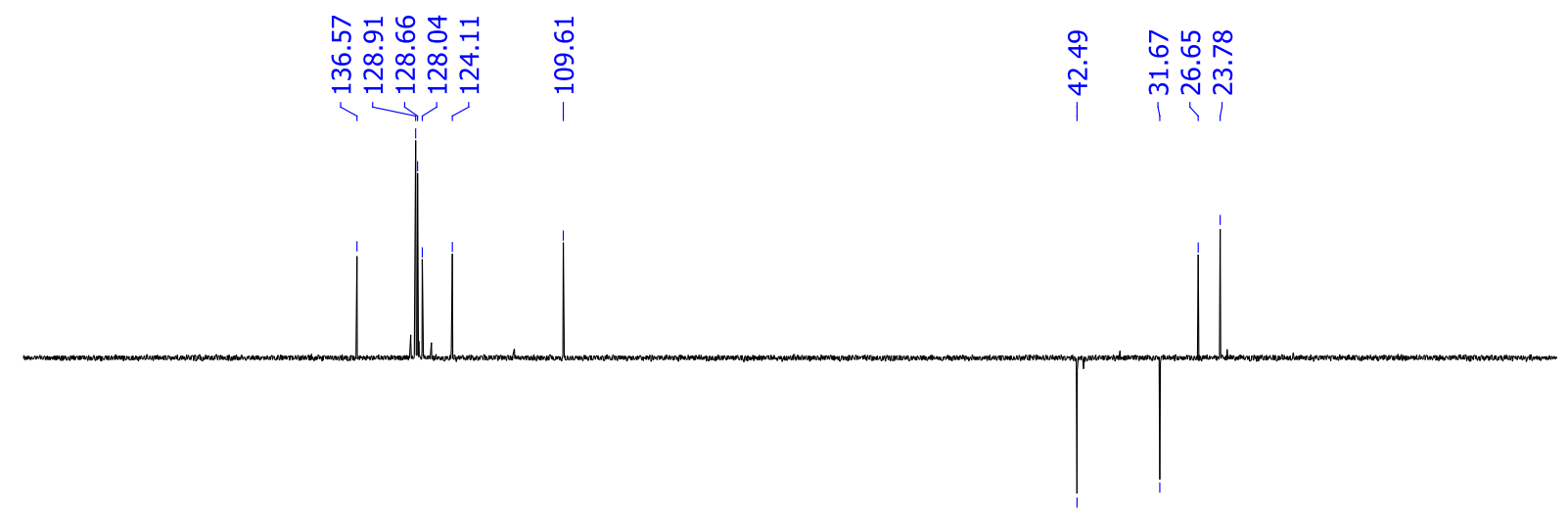

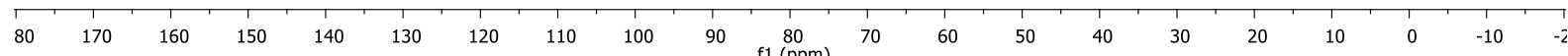


Copies of $\mathrm{H}^{1}, \mathrm{C}^{13}$ and DEPT 135 NMR spectra of compound 3ha

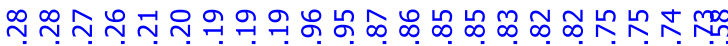

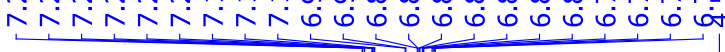

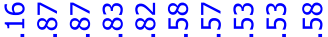<smiles>O=C1CC(=CC2C(=O)N3CCCC2C3)c2cc(F)ccc21</smiles>
min

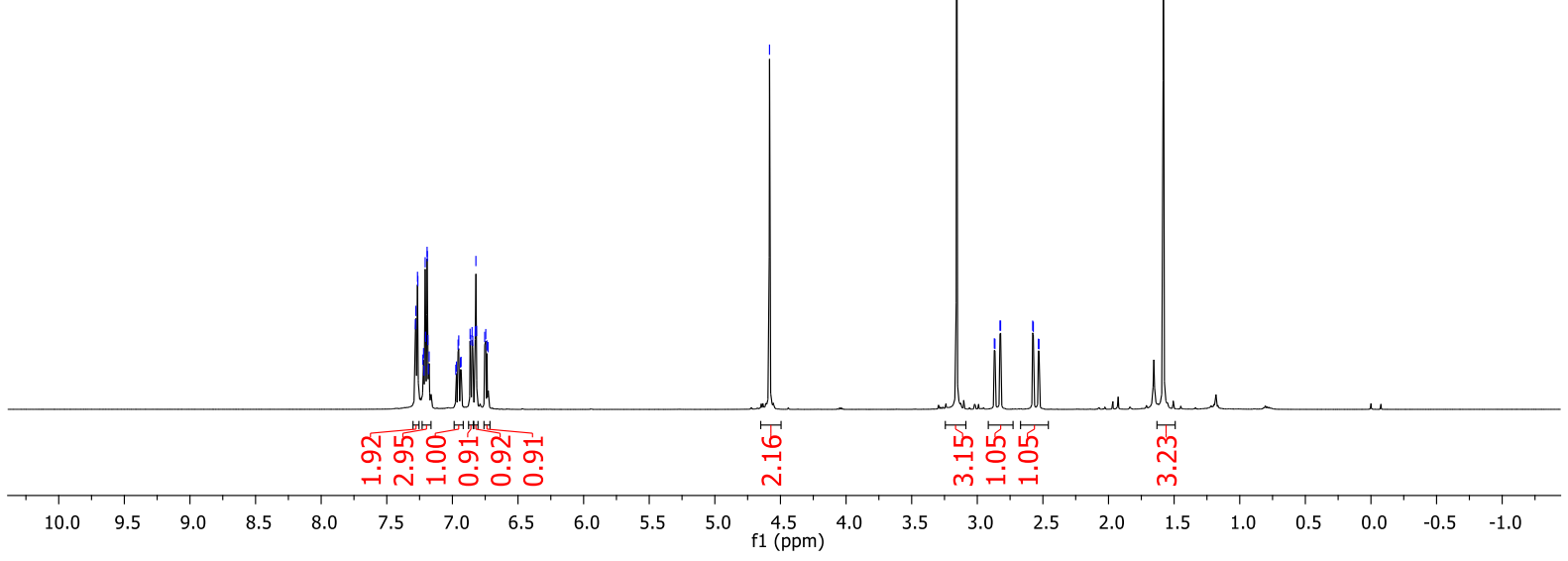

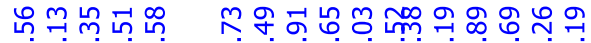

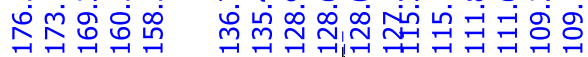

ㄷ.ㄴ.

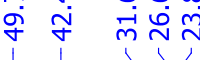

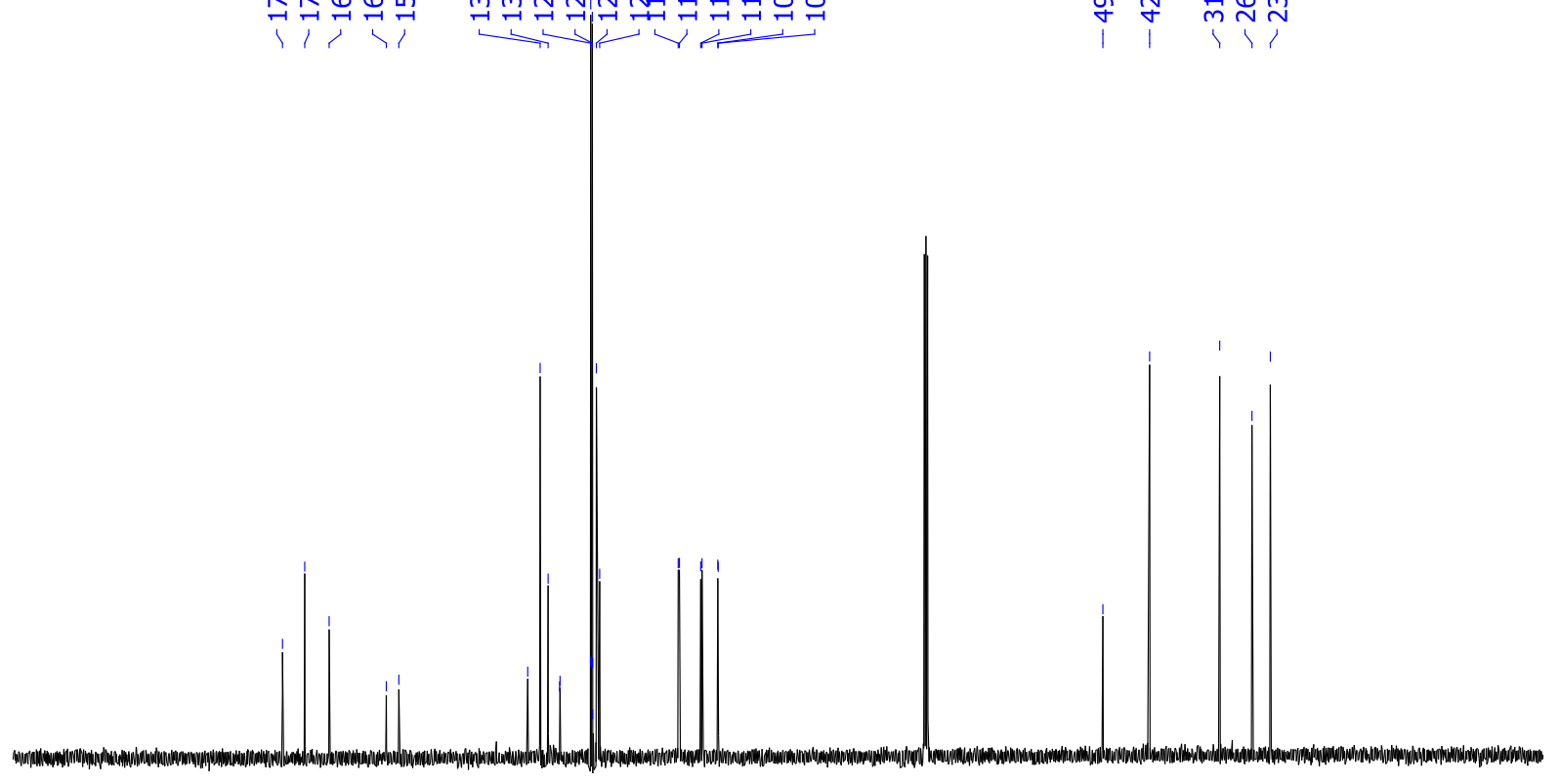

$\begin{array}{llllllllllllllllllllllll}210 & 200 & 190 & 180 & 170 & 160 & 150 & 140 & 130 & 120 & 110 & \underset{\mathrm{f} 1(\mathrm{ppm})}{100} & 90 & 80 & 70 & 60 & 50 & 40 & 30 & 20 & 10 & 0 & -10\end{array}$ 


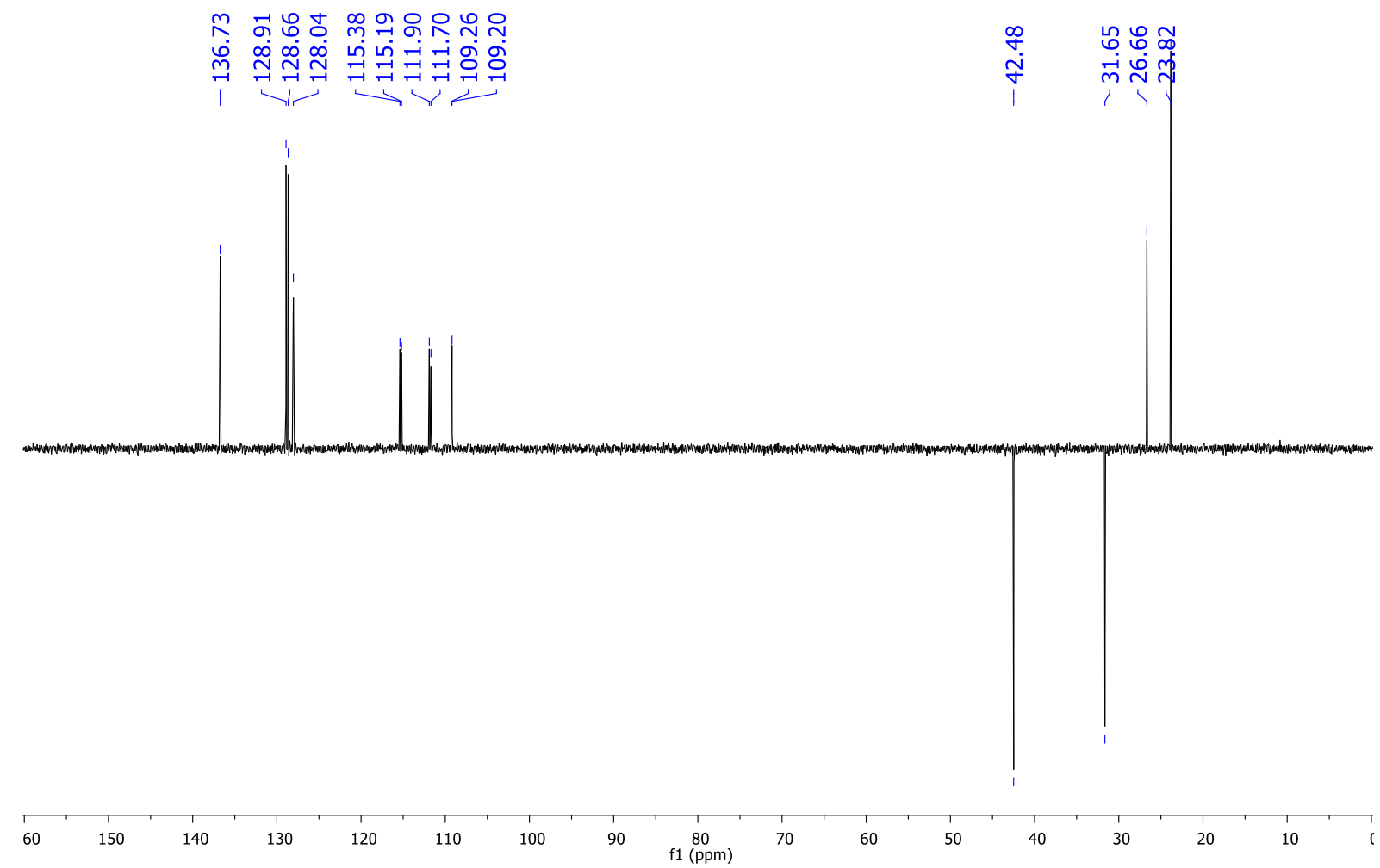


Copies of $\mathrm{H}^{1}, \mathrm{C}^{13}$ and DEPT 135 NMR spectra of compound 3ia

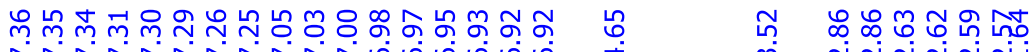

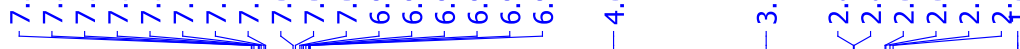
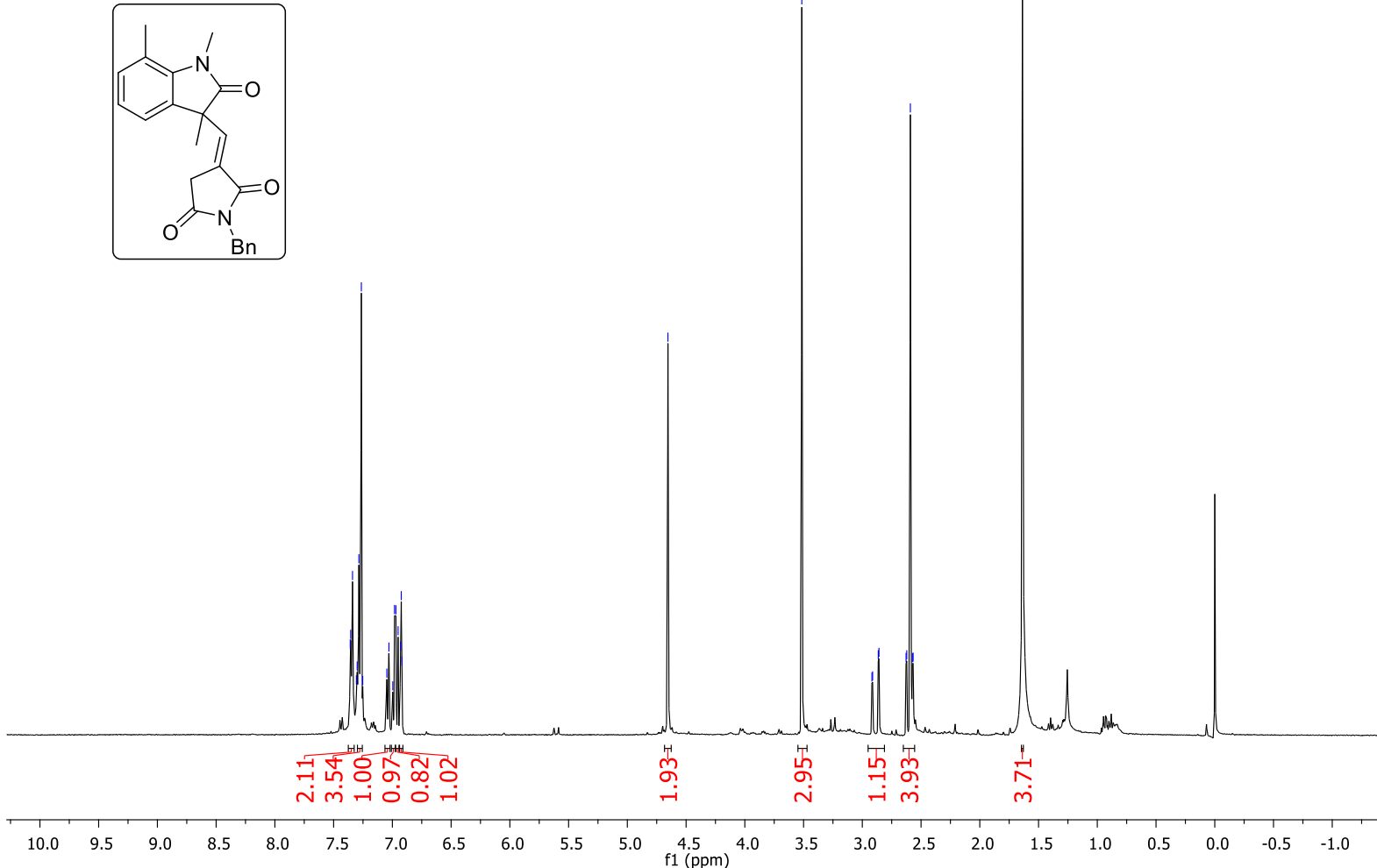

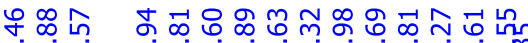

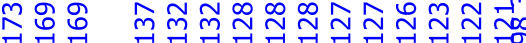

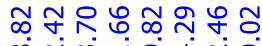

守

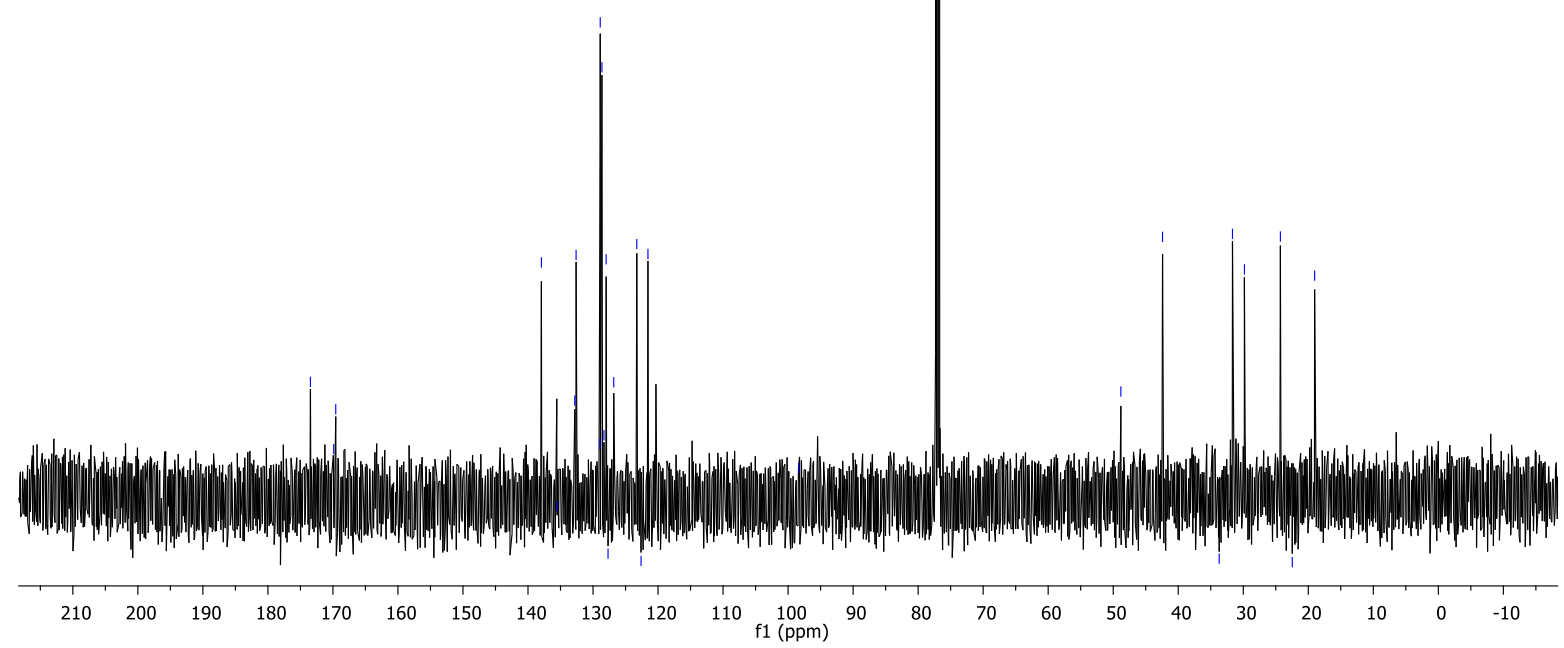




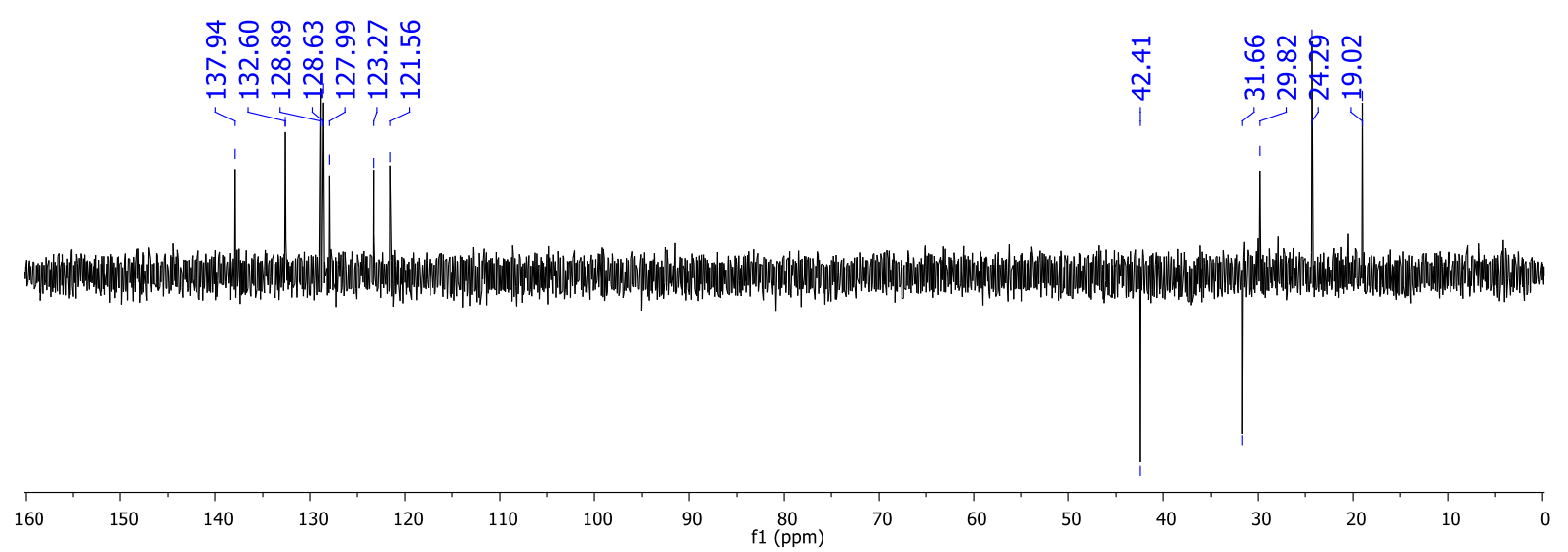


Copies of $\mathrm{H}^{1}, \mathrm{C}^{13}$ and DEPT 135 NMR spectra of compound $\mathbf{3 j a}$

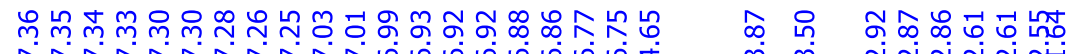

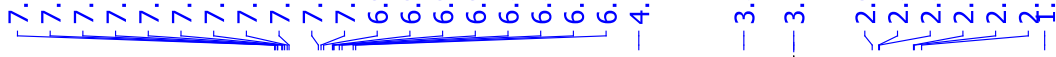

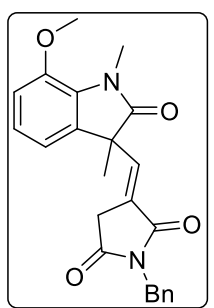

$\mathrm{Bn}$

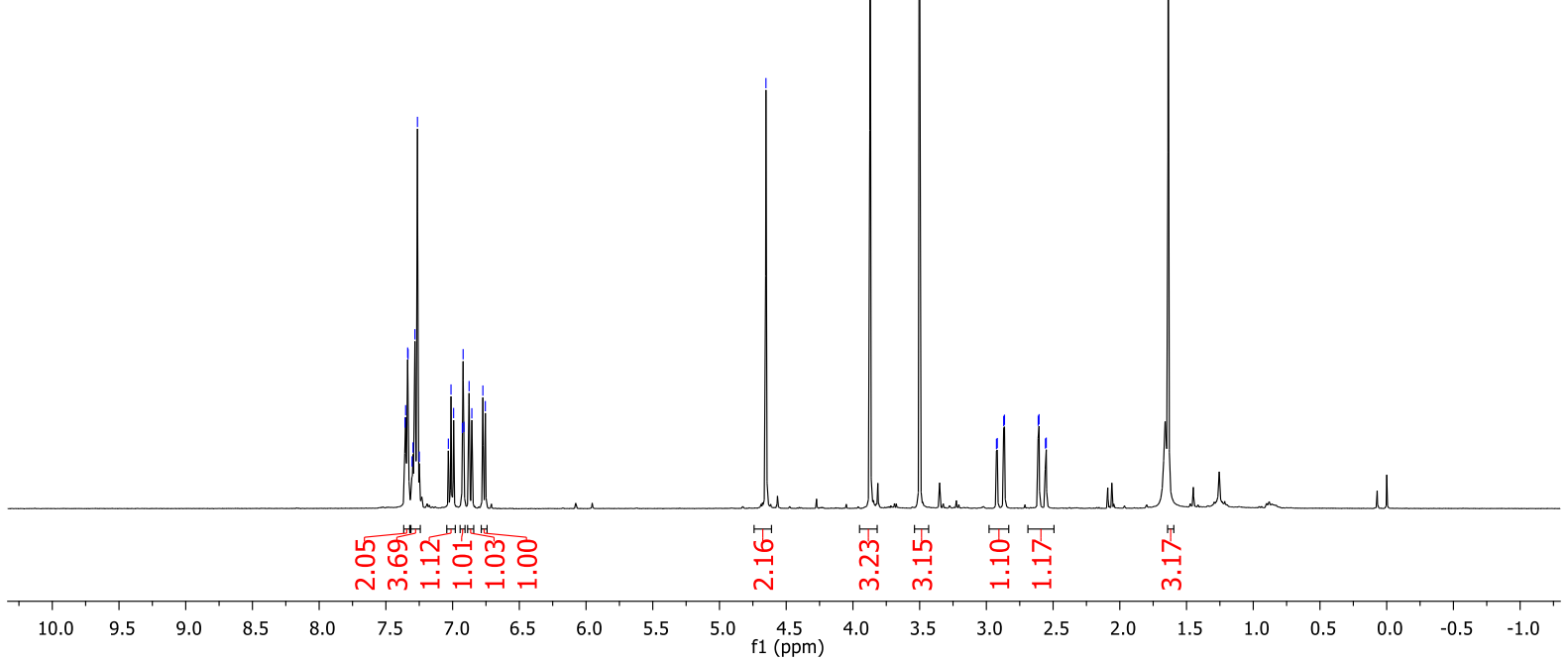

울ำน

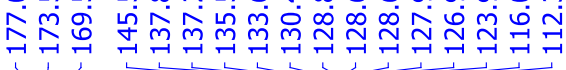

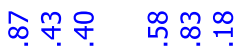

นก่์ ๆ

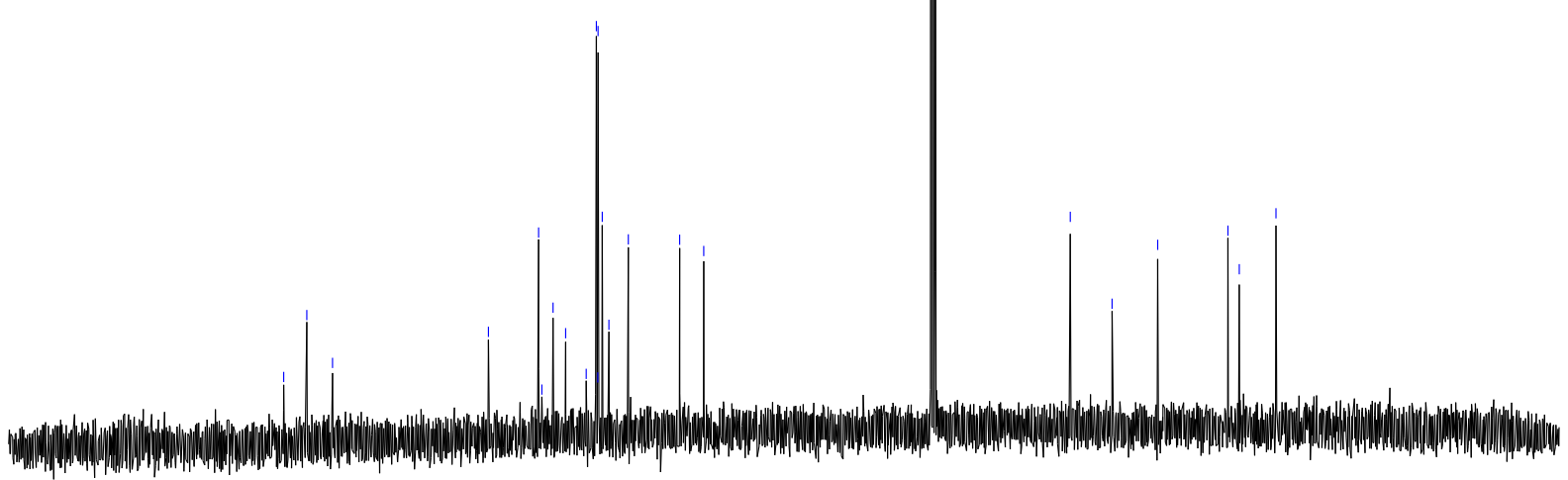

$\begin{array}{lllllllllllllllllllllll}210 & 200 & 190 & 180 & 170 & 160 & 150 & 140 & 130 & 120 & 110 & \begin{array}{c}100 \\ \mathrm{f} 1(\mathrm{ppm})\end{array} & 90 & 80 & 70 & 60 & 50 & 40 & 30 & 20 & 10 & 0 & -10\end{array}$ 


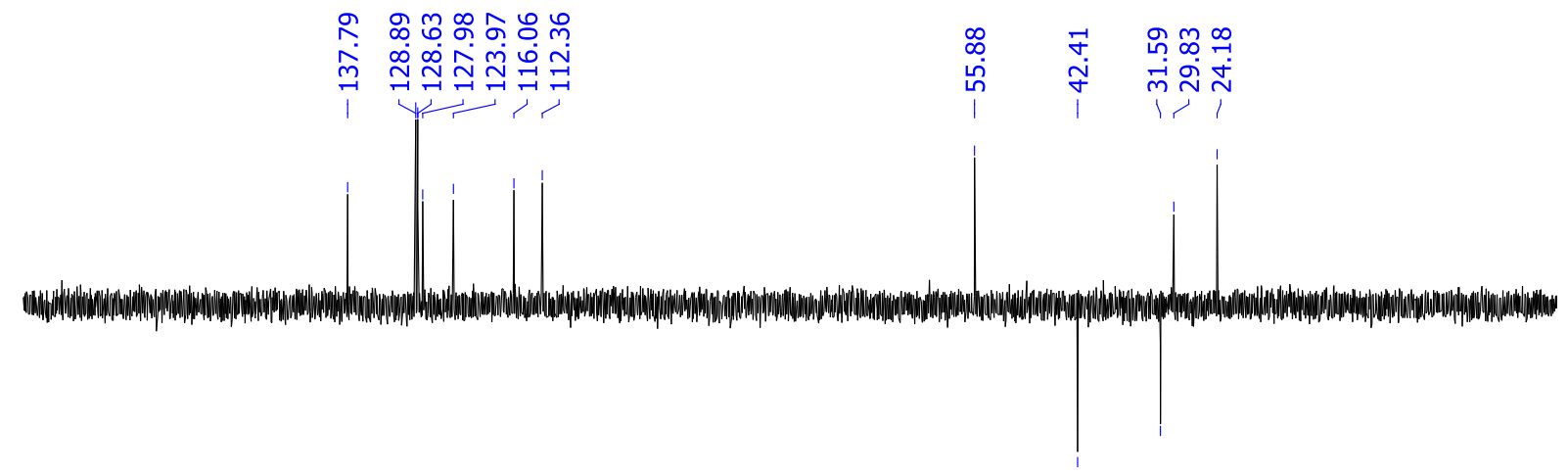

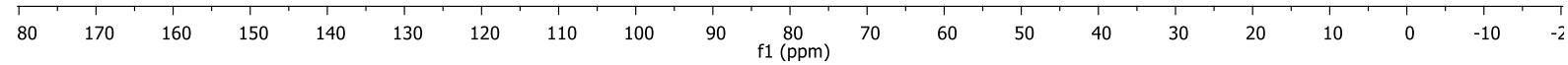


Copies of $\mathrm{H}^{1}, \mathrm{C}^{13}$ and DEPT 135 NMR spectra of compound $\mathbf{3 k a}$

లొ

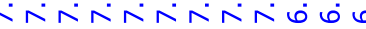

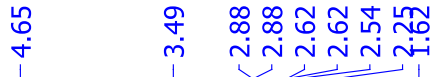

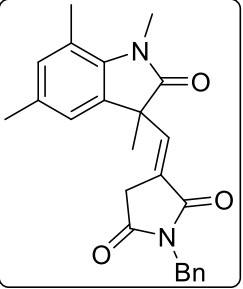

$\mathrm{Bn}$

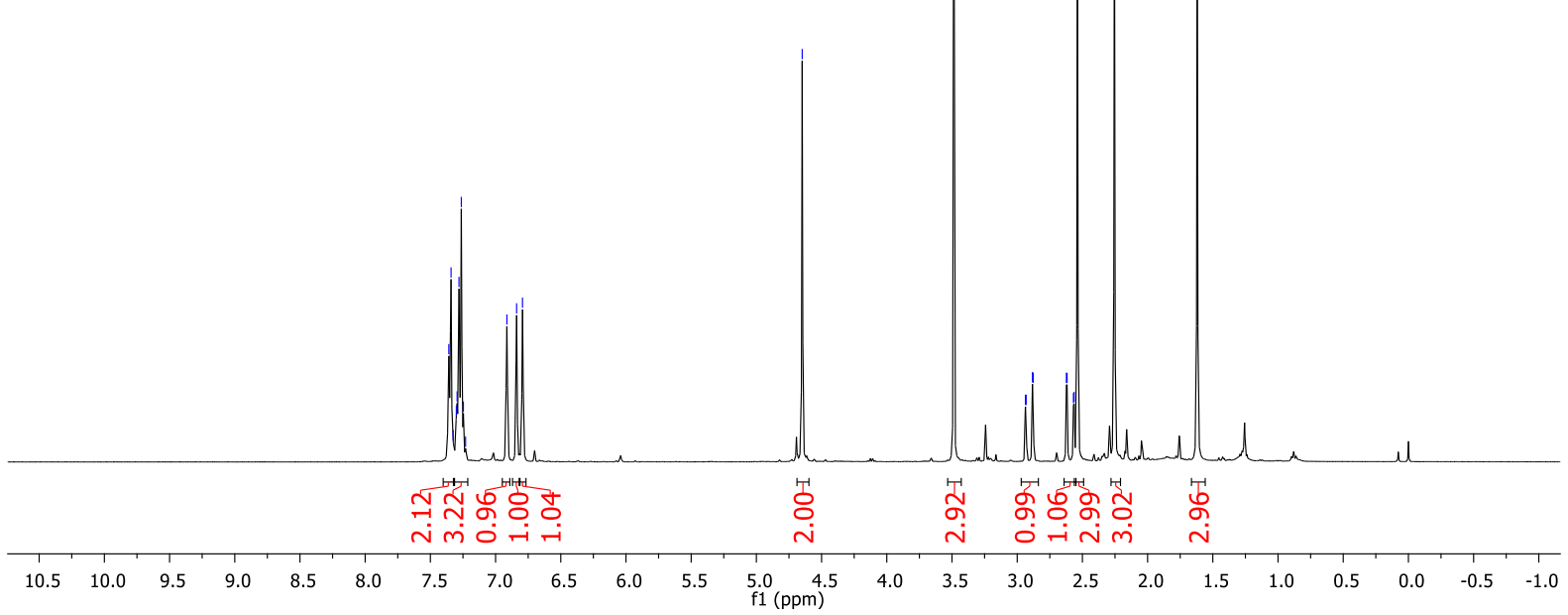

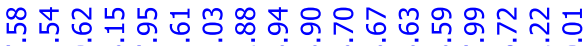

촉의

@ 우요에

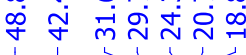

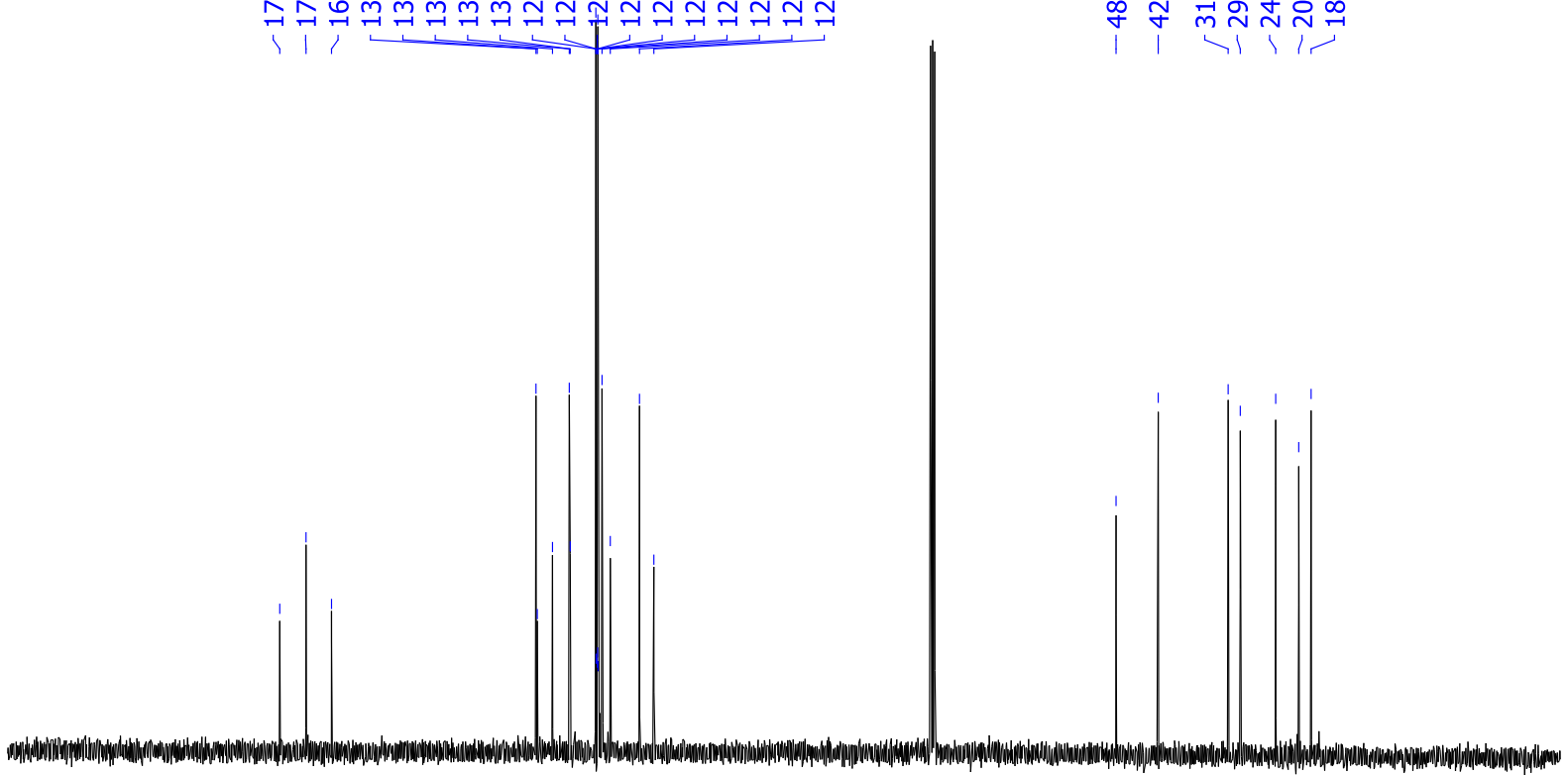

$\begin{array}{lllllllllllllllllllllllllll}210 & 200 & 190 & 180 & 170 & 160 & 150 & 140 & 130 & 120 & 110 & 100 & 90 & 80 & 70 & 60 & 50 & 40 & 30 & 20 & 10 & 0 & -10 & \end{array}$ 


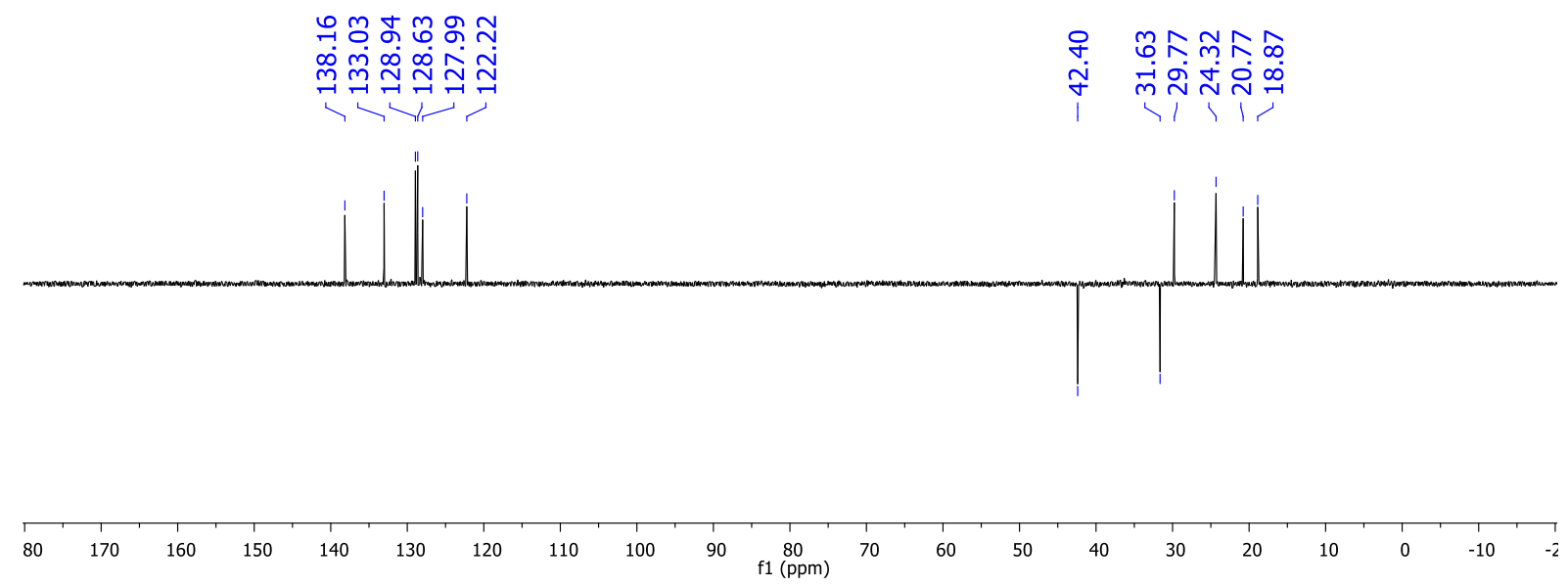


Copies of $\mathrm{H}^{1}, \mathrm{C}^{13}$ and DEPT 135 NMR spectra of compound 3la

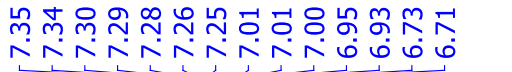

$\underset{\sim}{\mathscr{0}}$

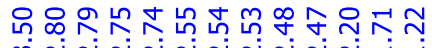

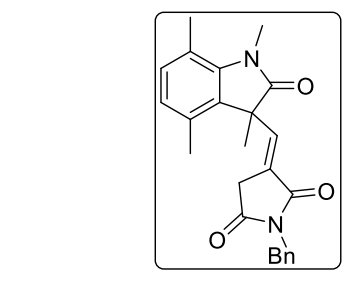

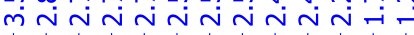

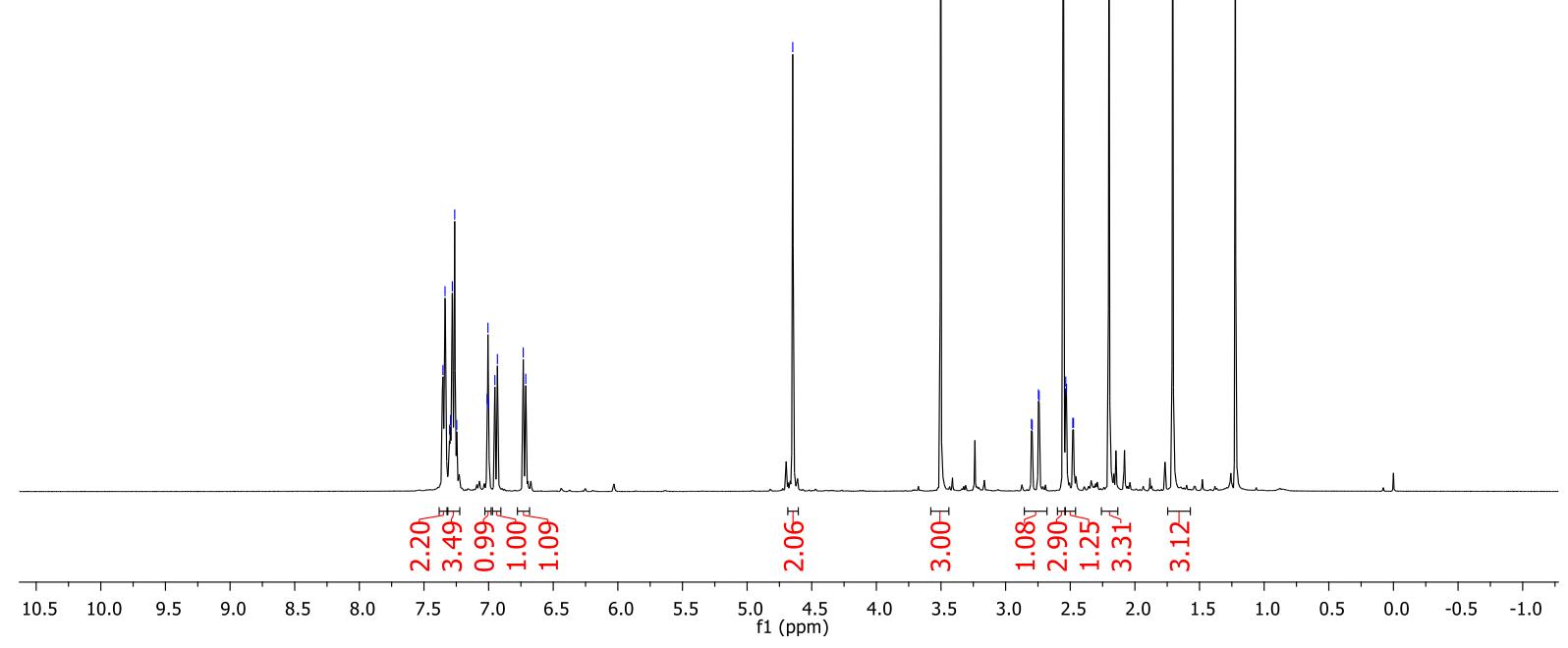

불 m

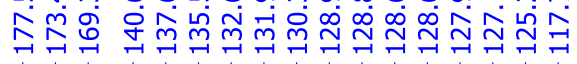

กี 굼

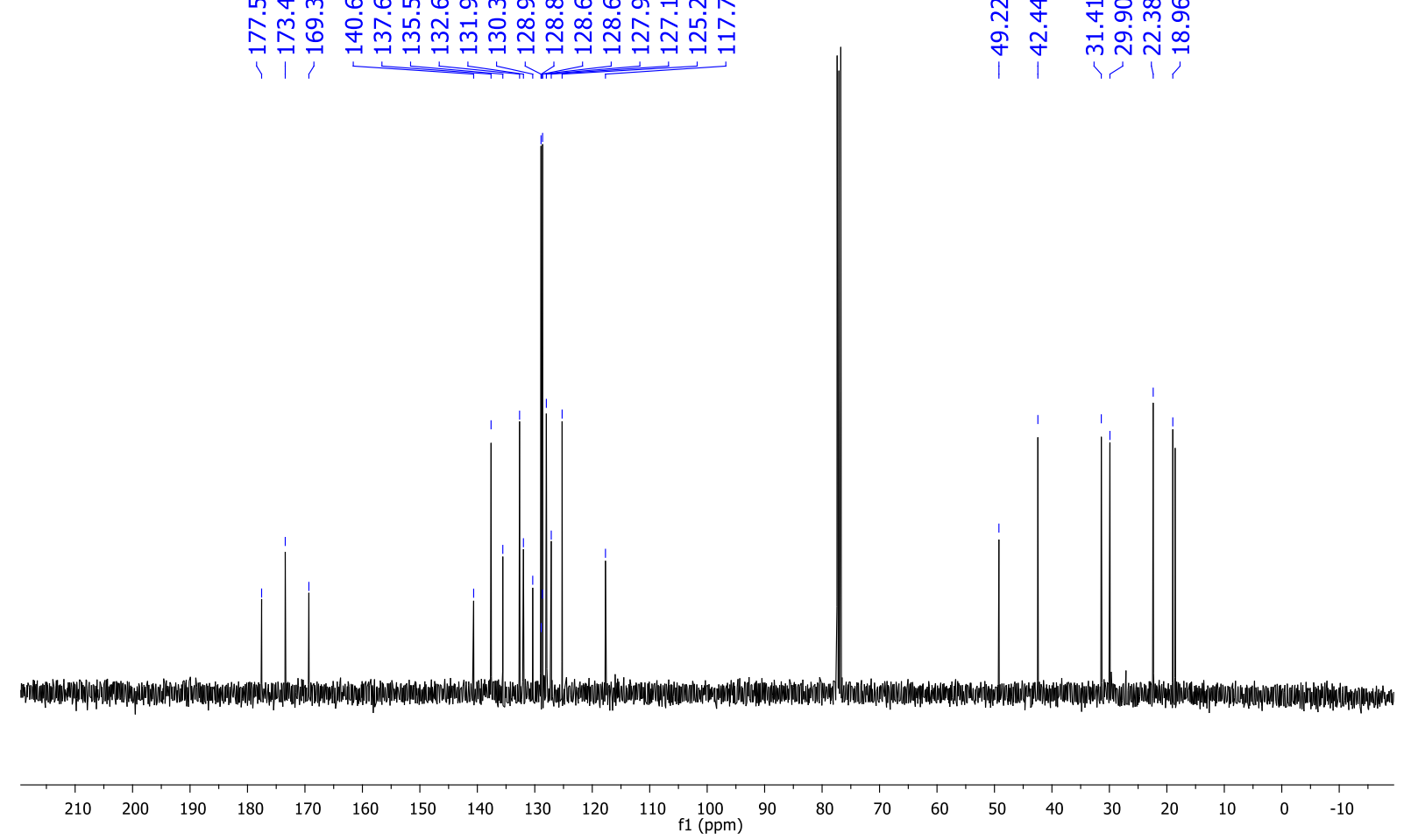




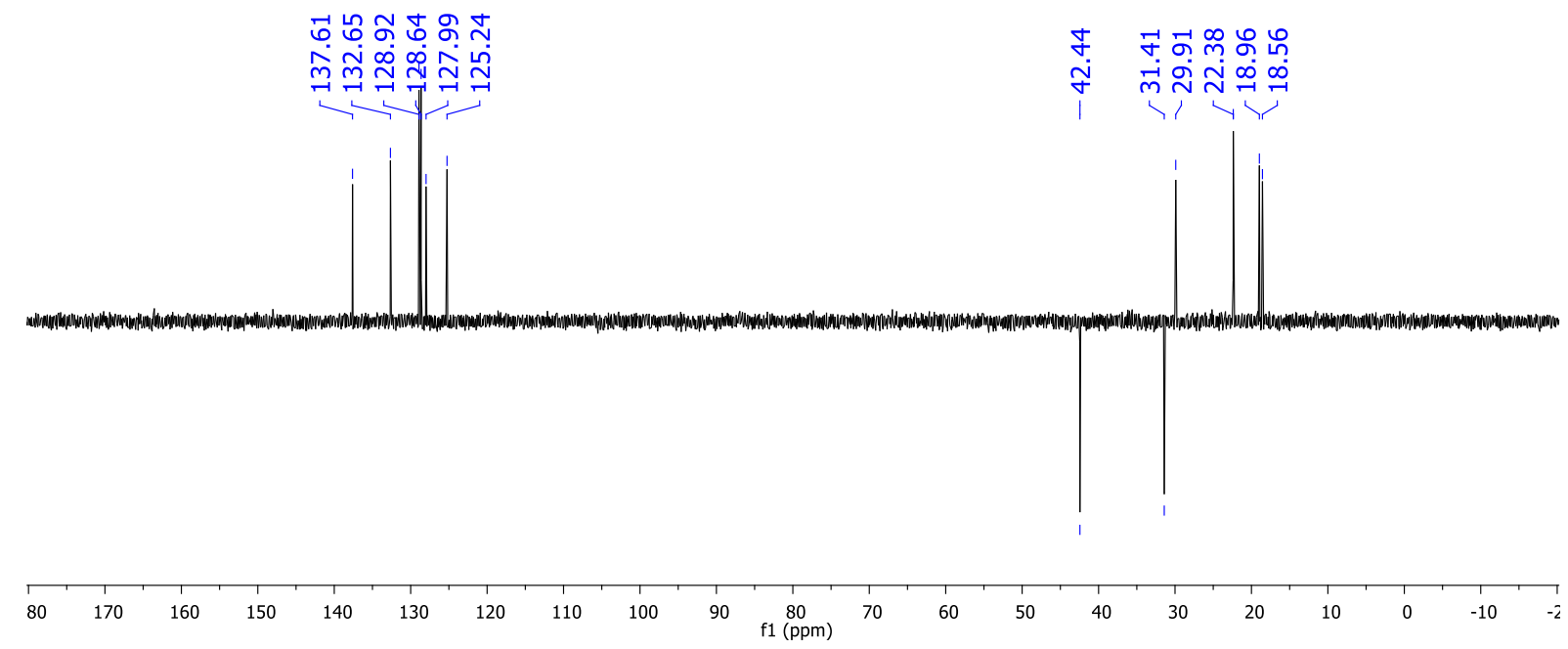


Copies of $\mathrm{H}^{1}, \mathrm{C}^{13}$ and DEPT $135 \mathrm{NMR}$ spectra of compound 3ma

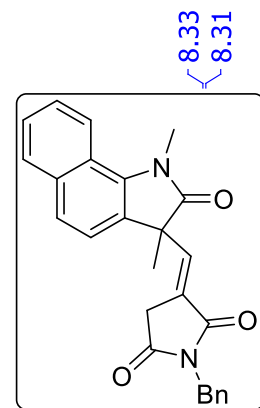

กิ

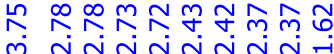

I $\sqrt{2 \pi n} \sqrt{2} \sin$

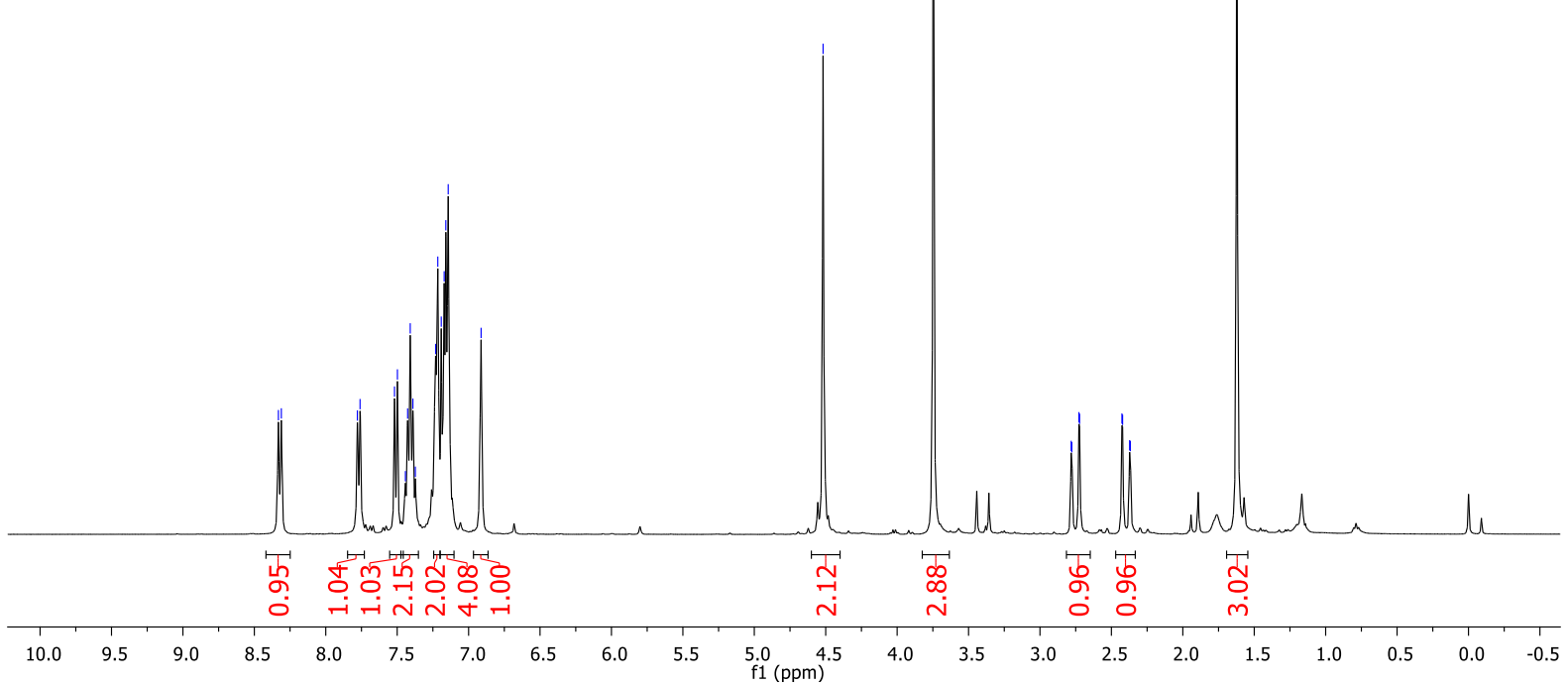

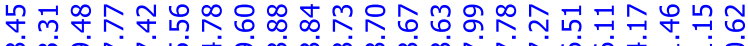

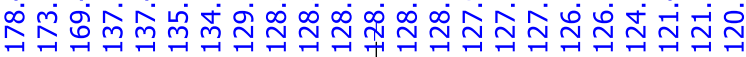

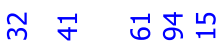

ค

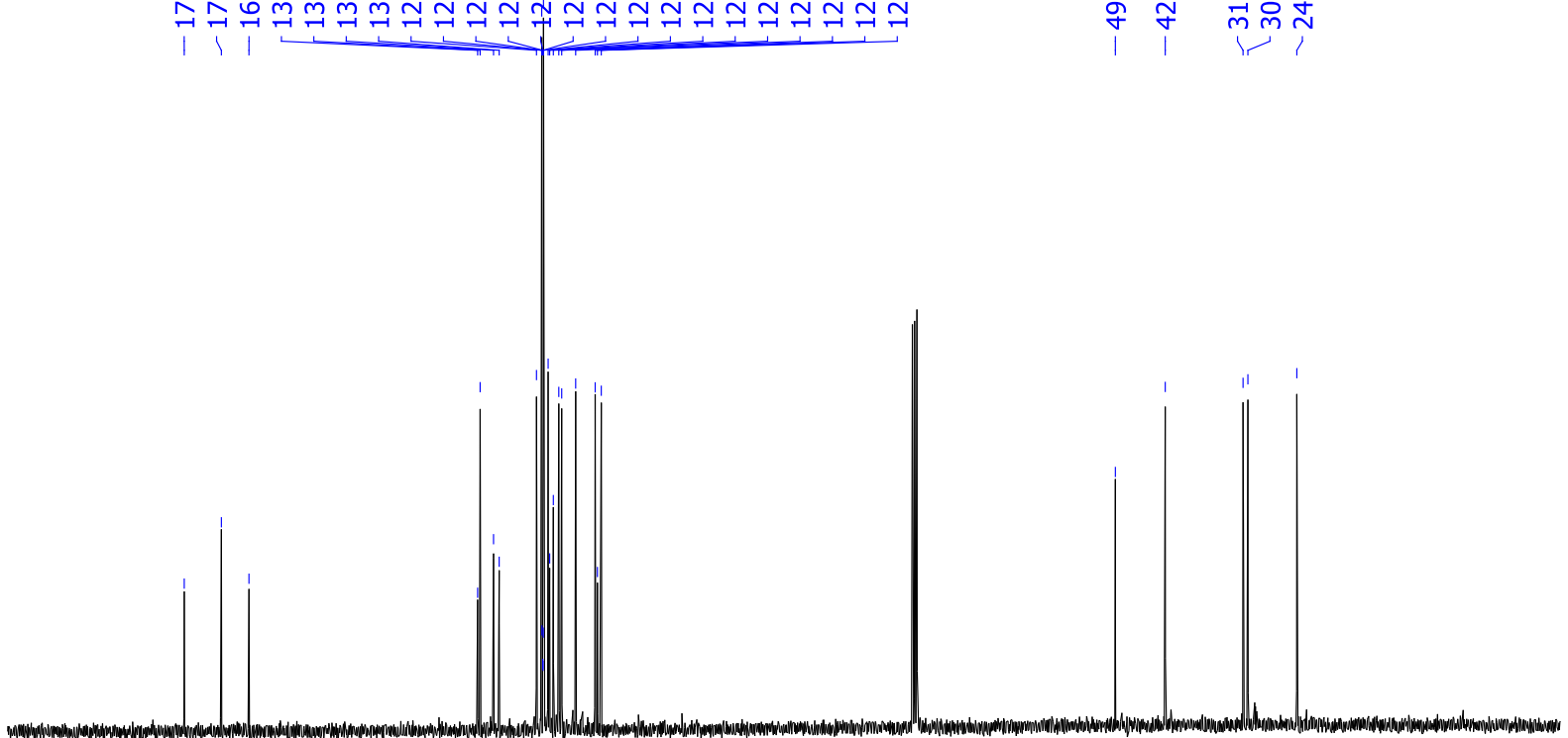

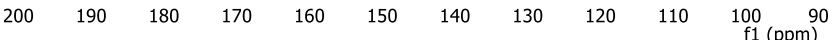




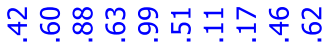

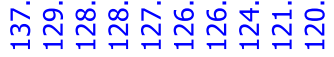

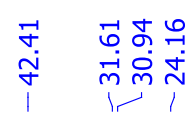
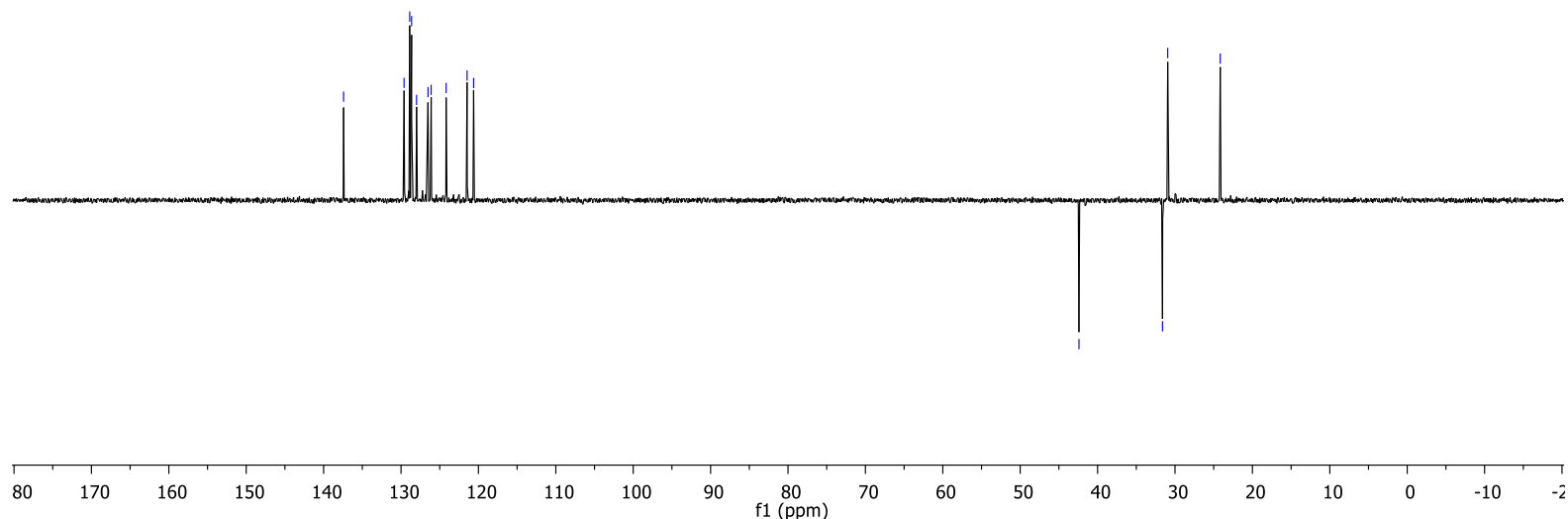
Copies of $\mathrm{H}^{1}, \mathrm{C}^{13}$ and DEPT 135 NMR spectra of compound 3na
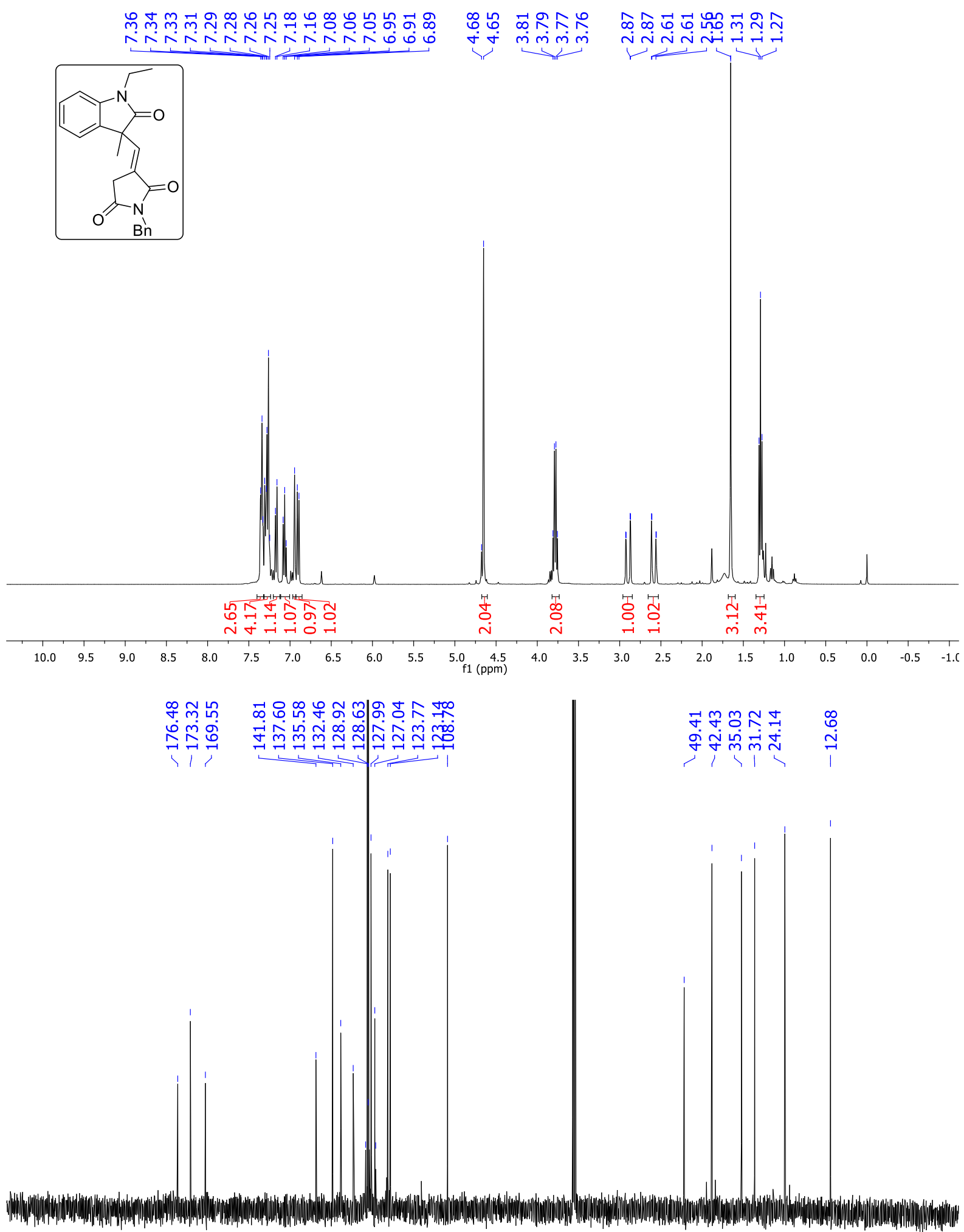

$\begin{array}{lllllllllllllllllllllll}210 & 200 & 190 & 180 & 170 & 160 & 150 & 140 & 130 & 120 & 110 & 100 & 90 & 80 & 70 & 60 & 50 & 40 & 30 & 20 & 10 & 0 & -10\end{array}$ 


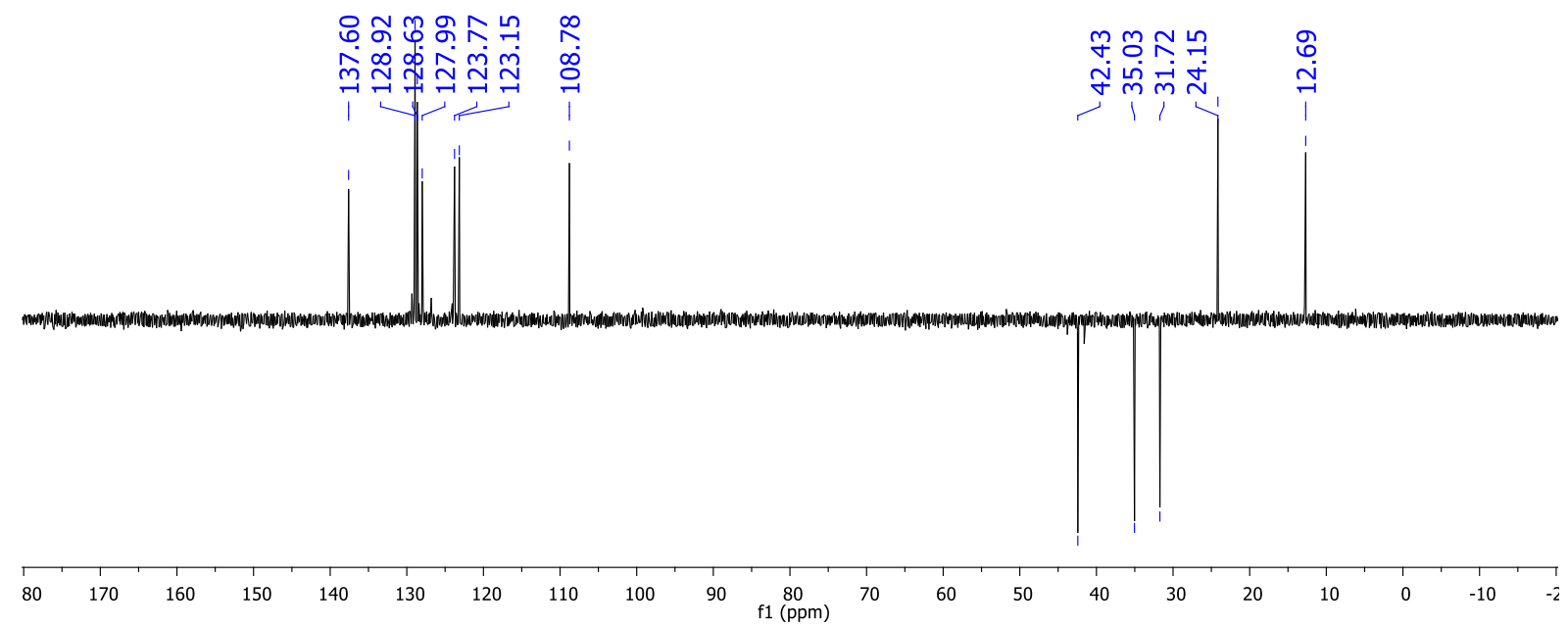


Copies of $\mathrm{H}^{1}, \mathrm{C}^{13}$ and DEPT 135 NMR spectra of compound 3oa

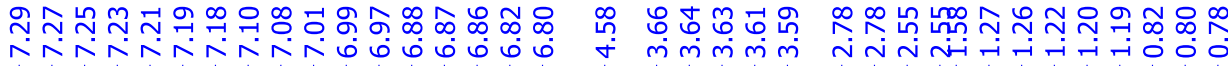
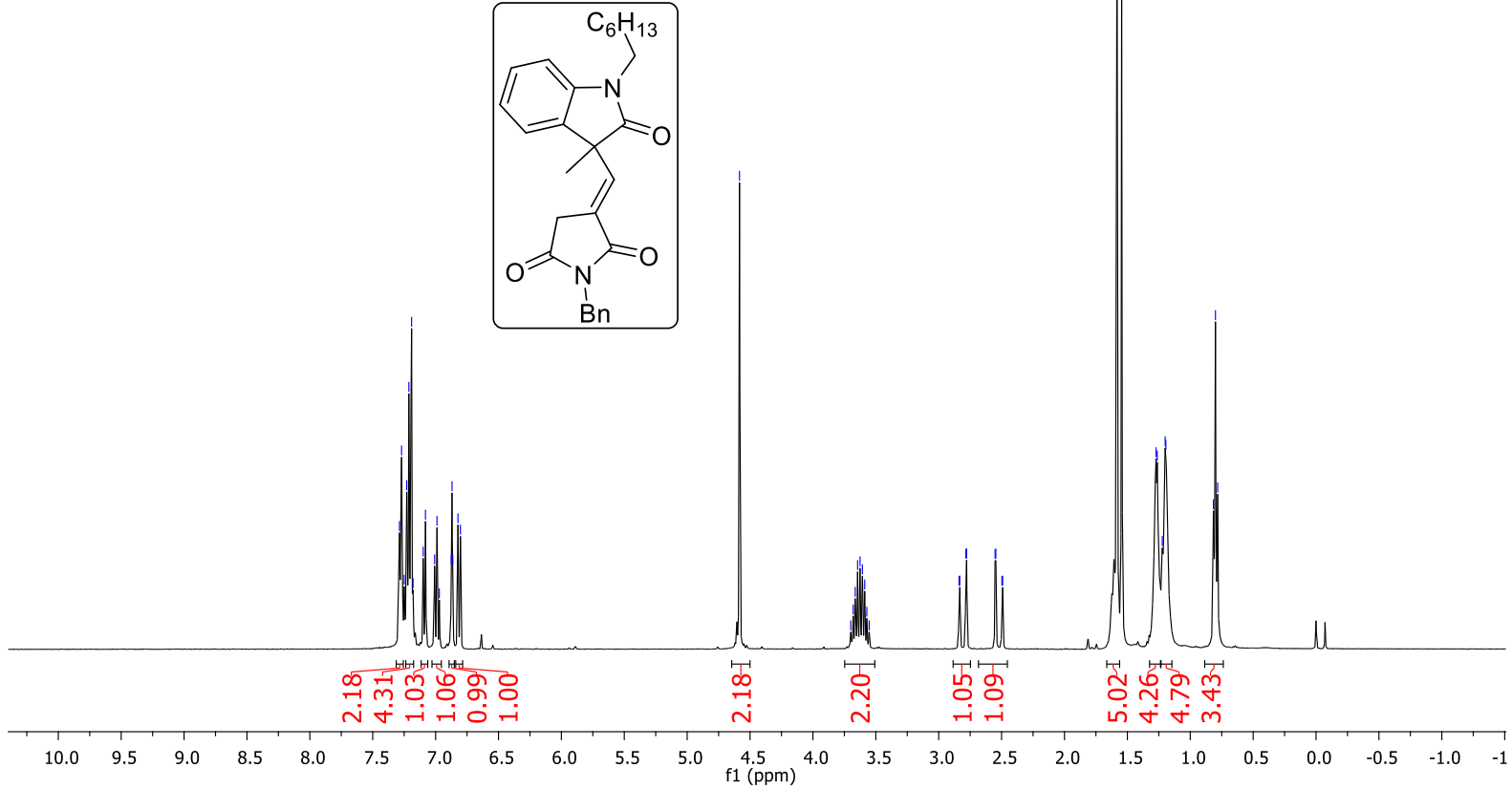

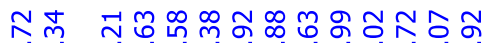

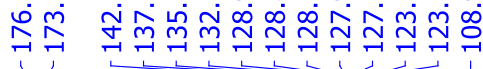

웅ํำ

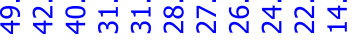

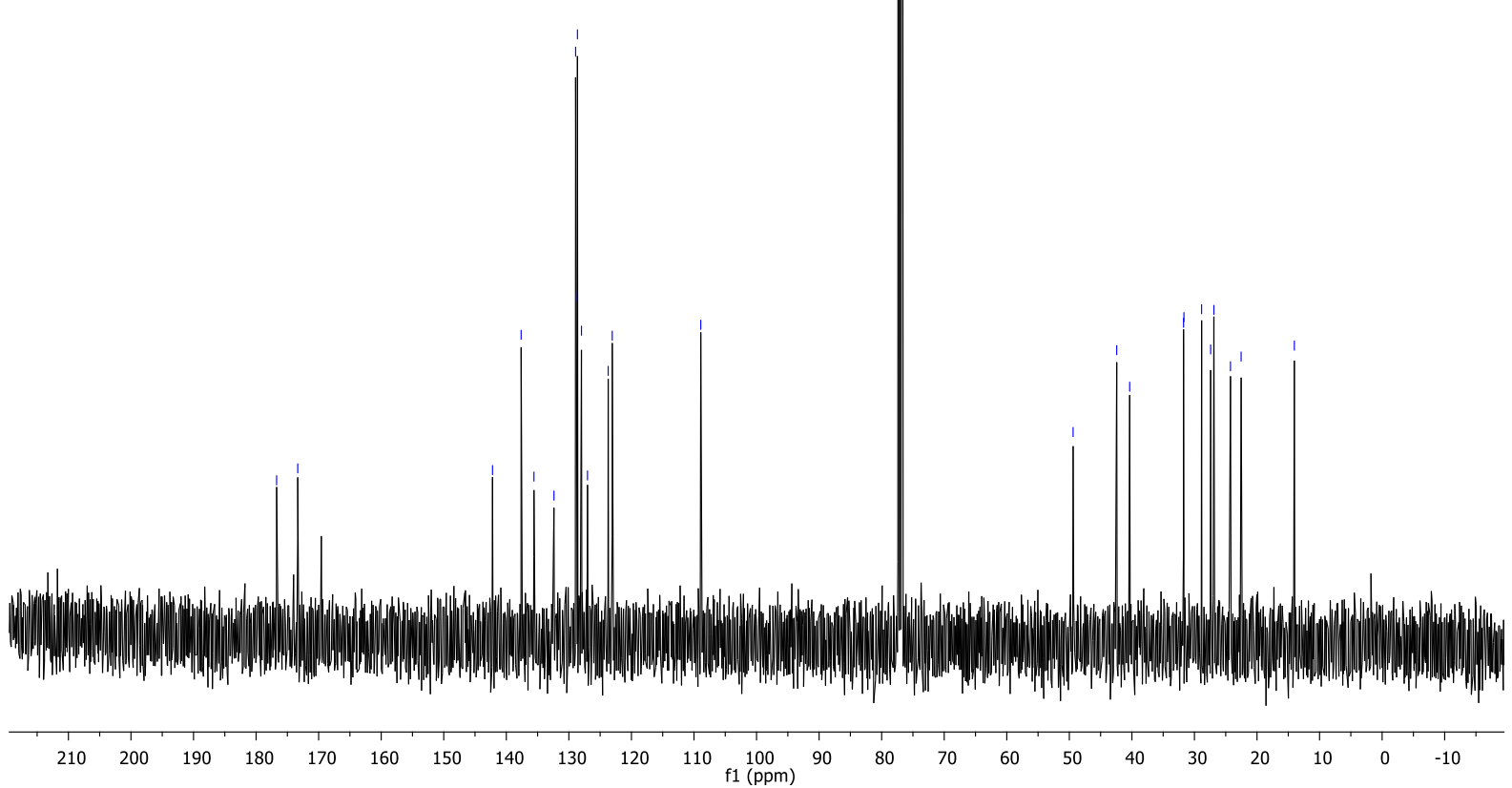




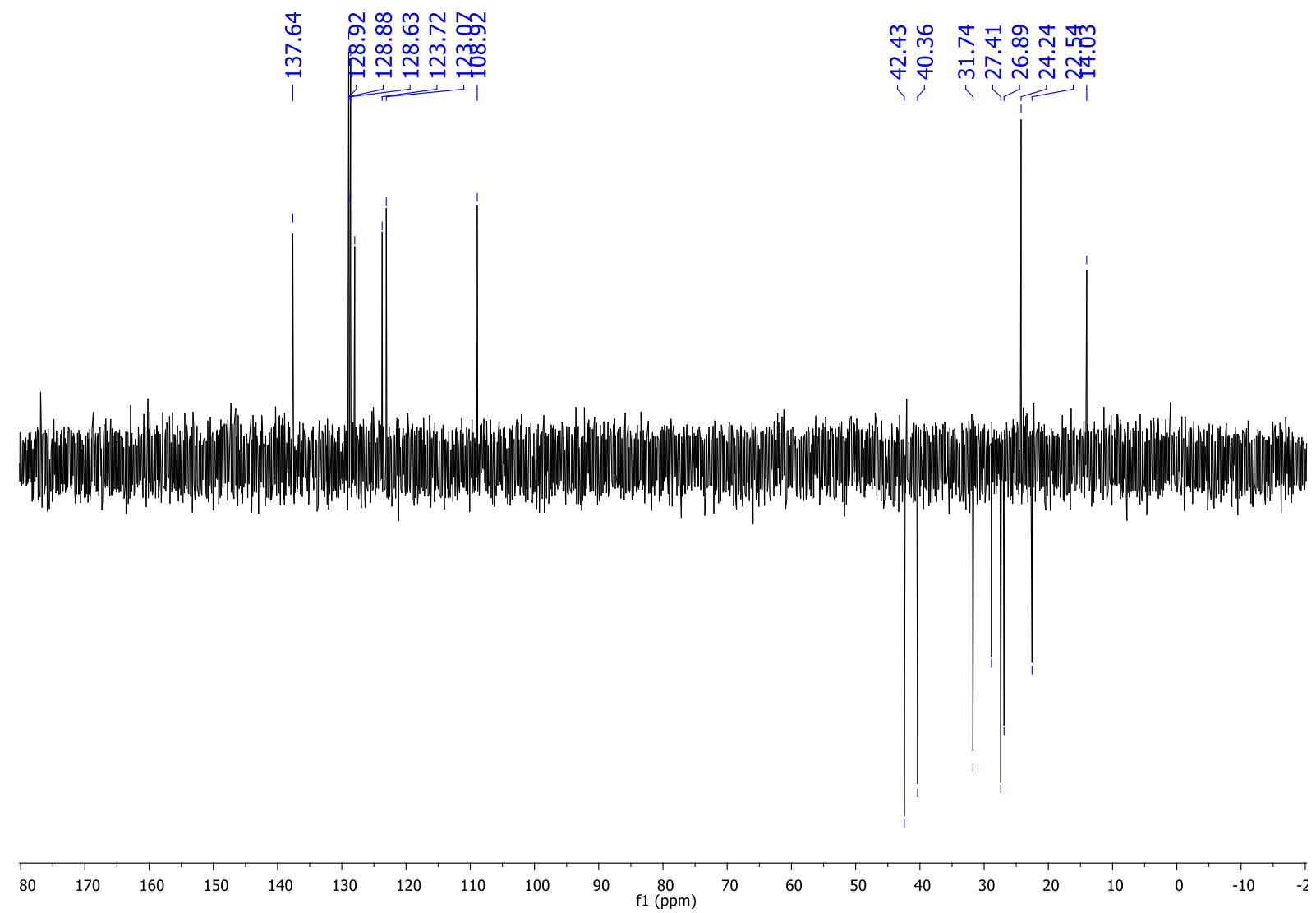


Copies of $\mathrm{H}^{1}, \mathrm{C}^{13}$ and DEPT 135 NMR spectra of compound $\mathbf{3 p a}$

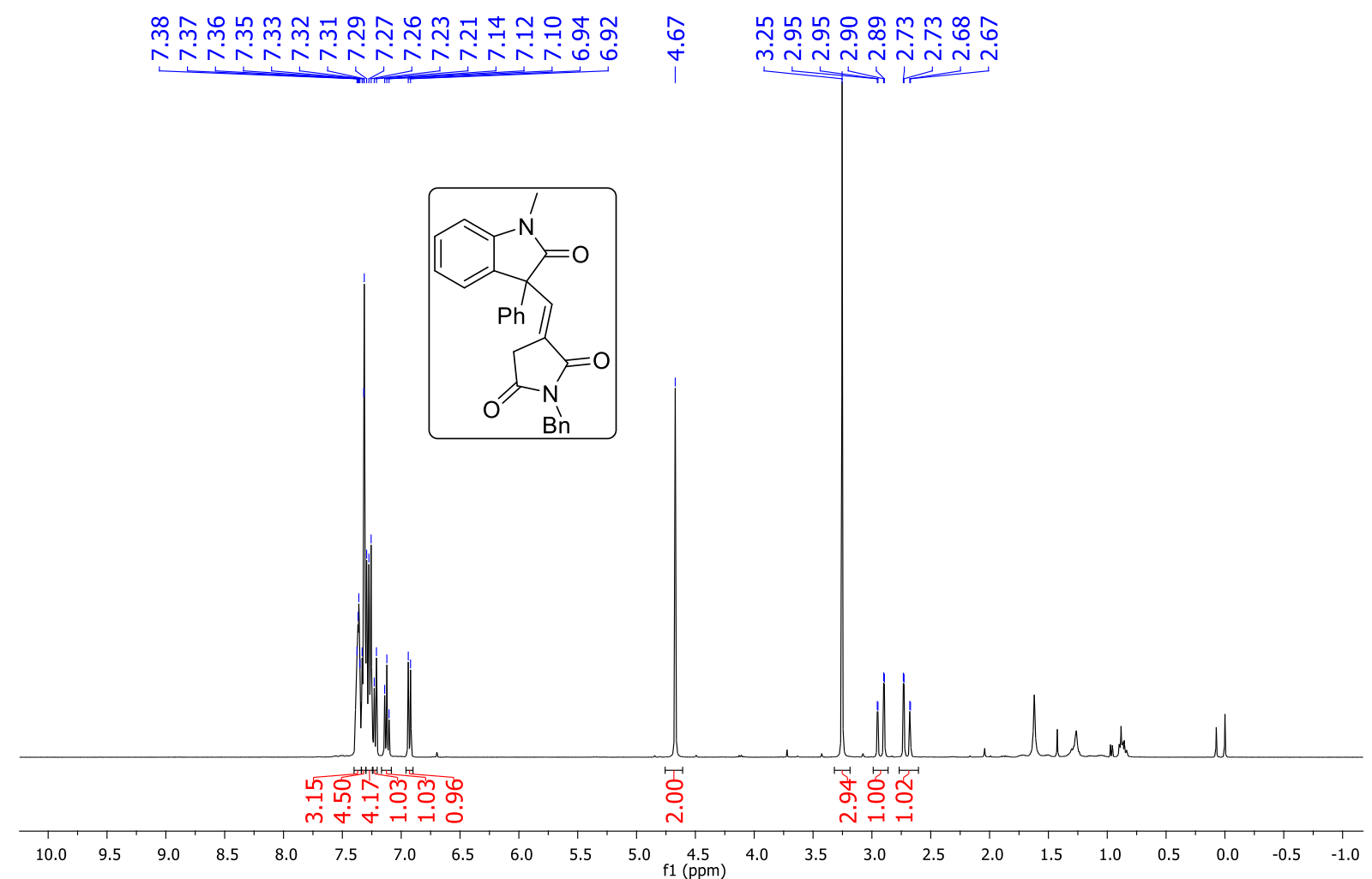

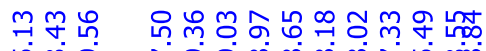

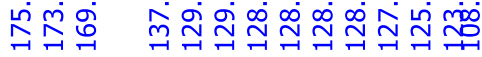

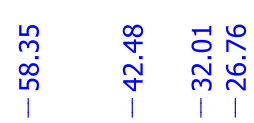

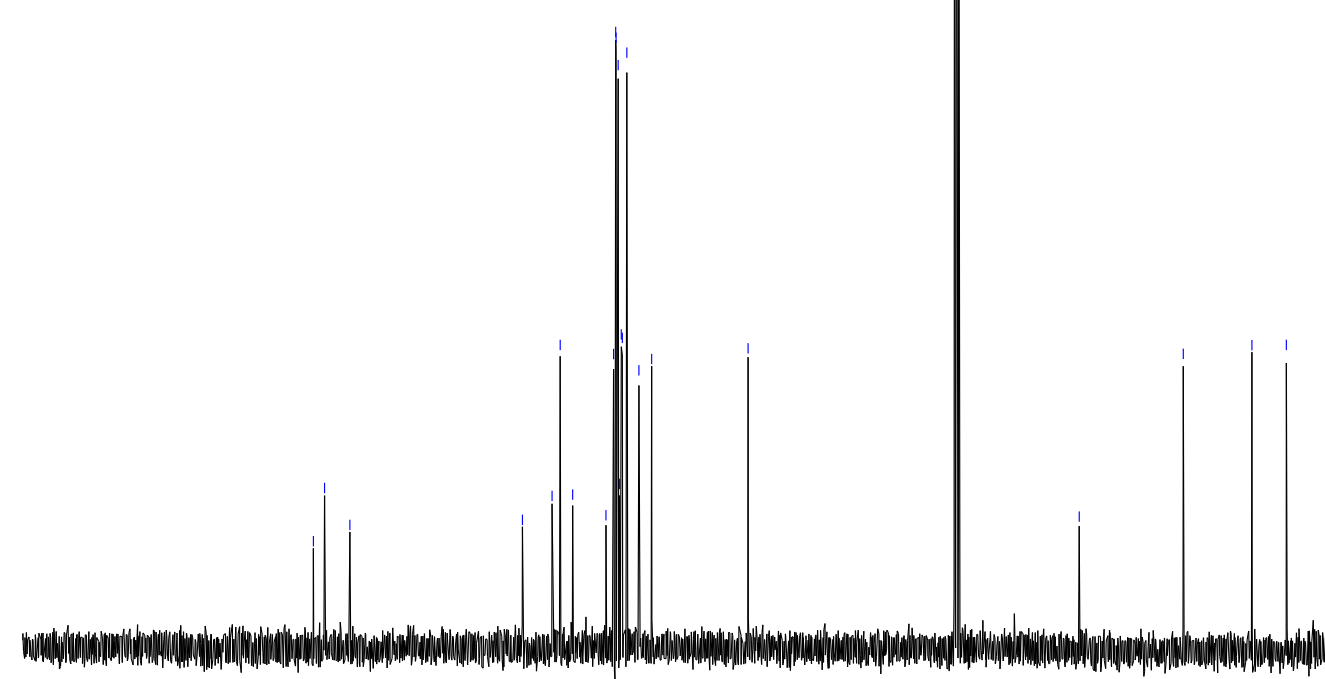

$\begin{array}{llllllllllllllllllllll}210 & 200 & 190 & 180 & 170 & 160 & 150 & 140 & 130 & 120 & 110 \underset{\mathrm{f} 1(\mathrm{ppm})}{100} & 90 & 80 & 70 & 60 & 50 & 40 & 30 & 20 & 10 & 0 & -10\end{array}$ 


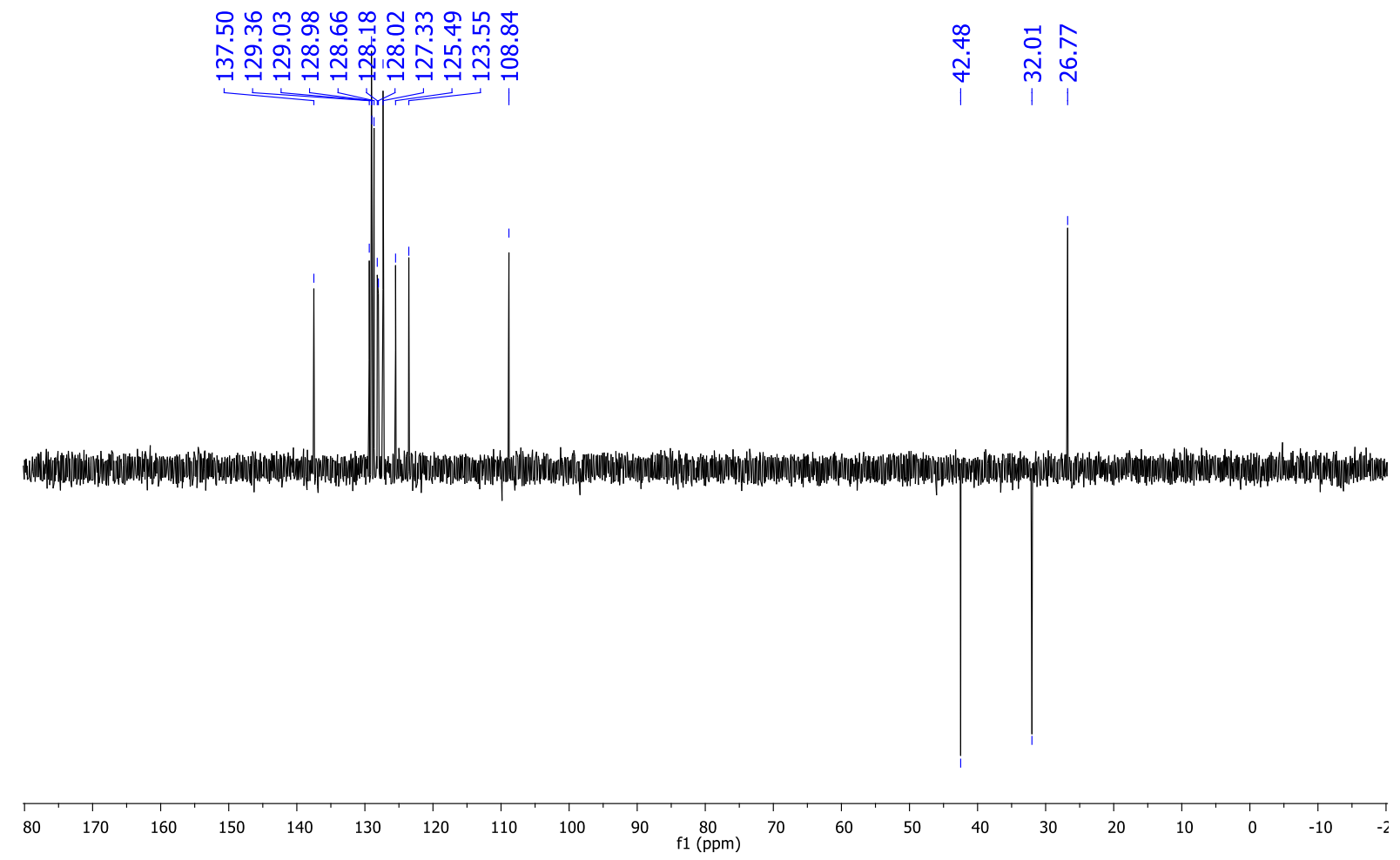


Copies of $\mathrm{H}^{1}, \mathrm{C}^{13}$ and DEPT 135 NMR spectra of compound 3qa

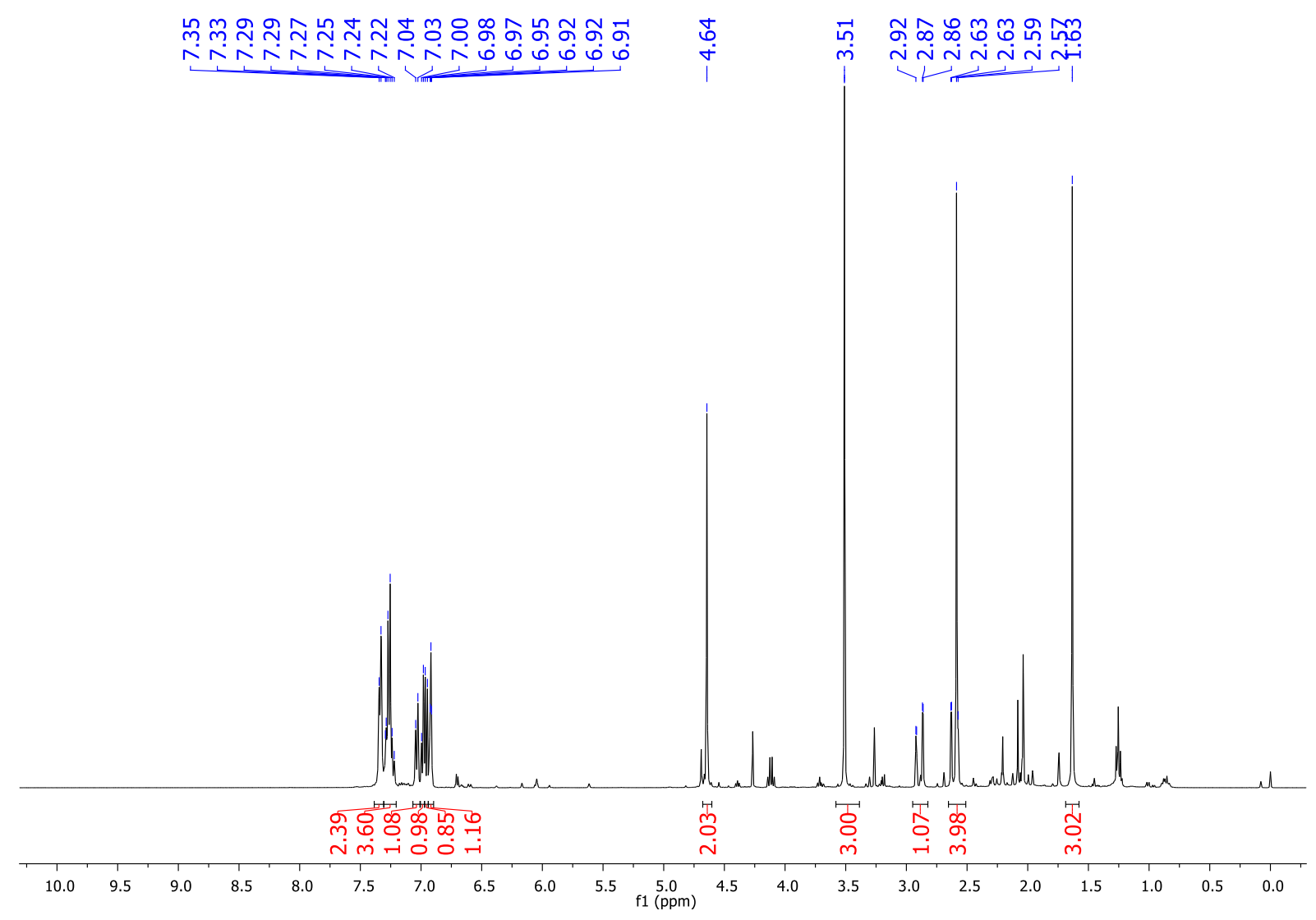

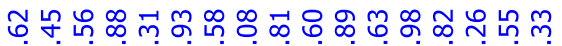

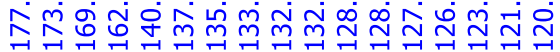

¿

文

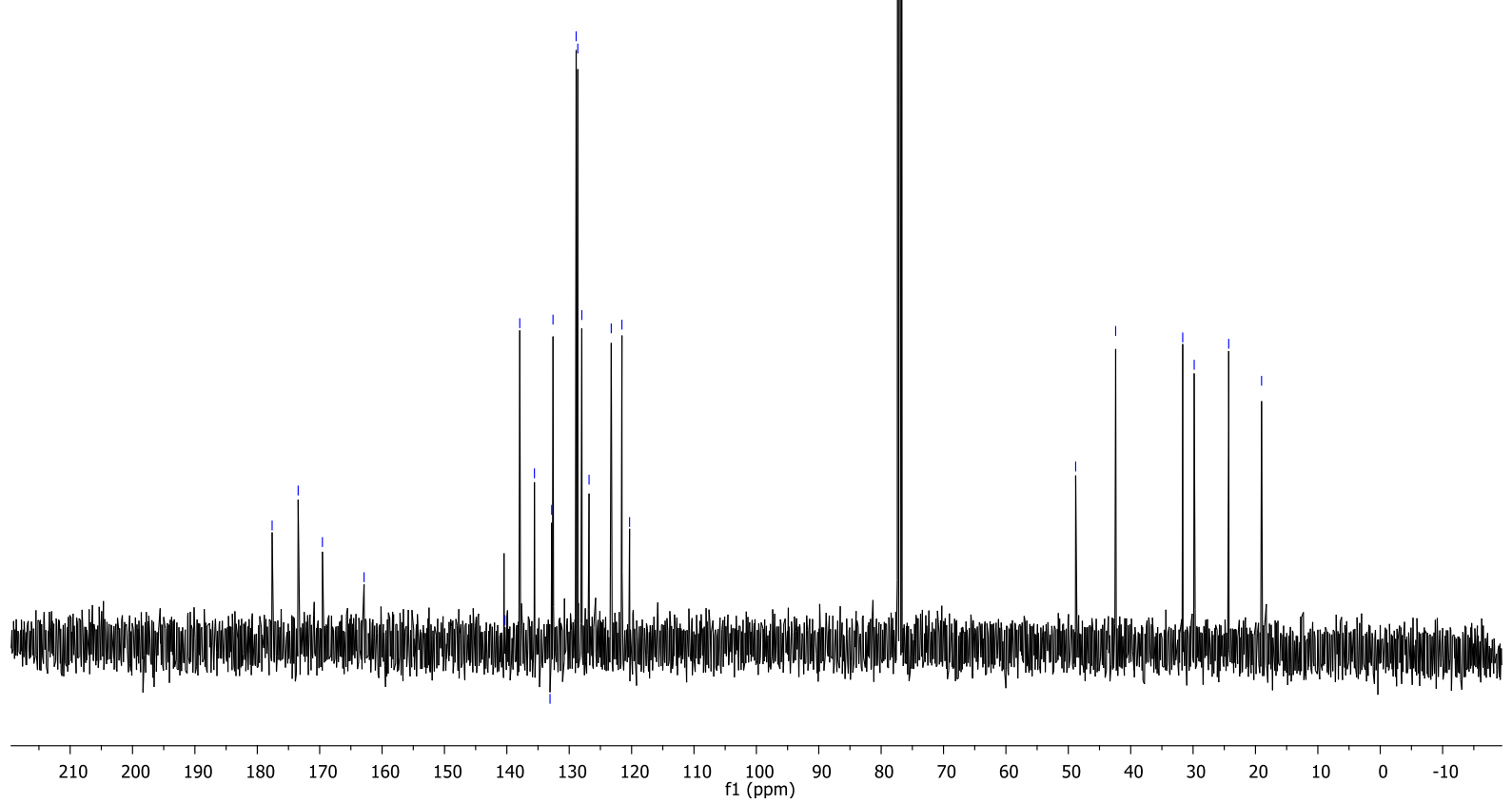




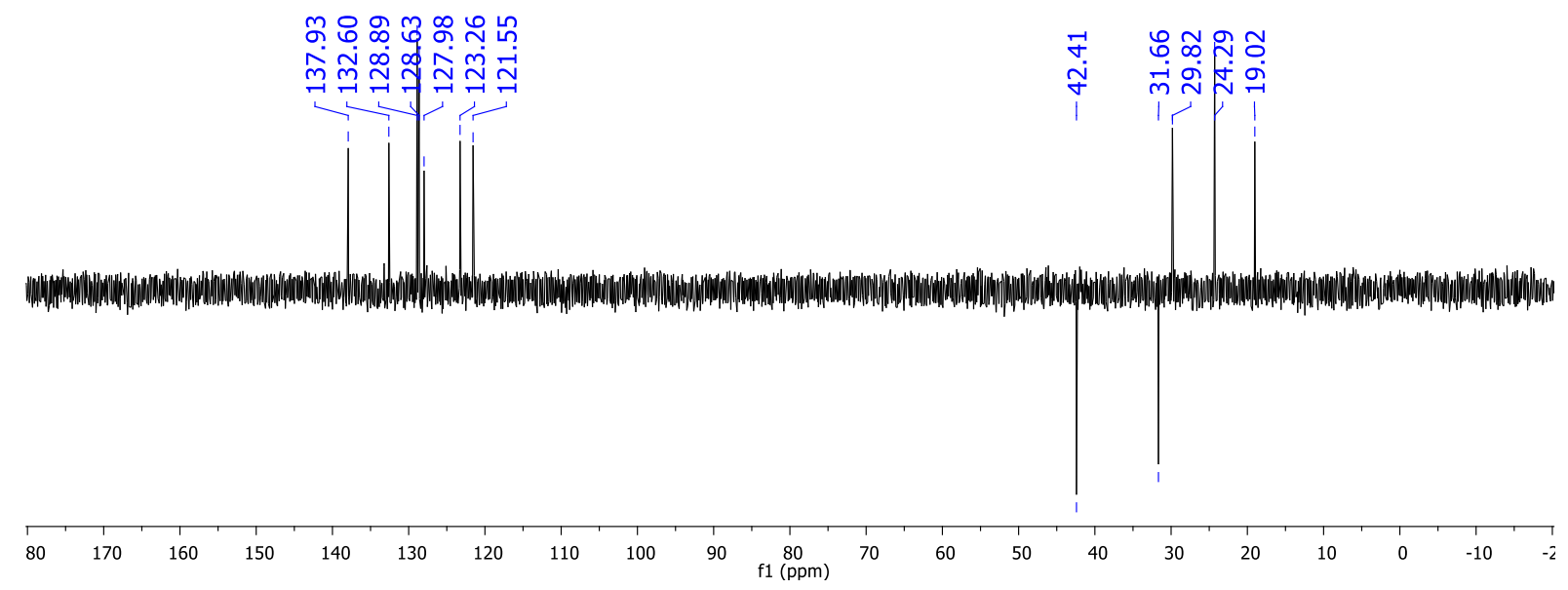


Copies of $\mathrm{H}^{1}, \mathrm{C}^{13}$ and DEPT 135 NMR spectra of compound 3ra

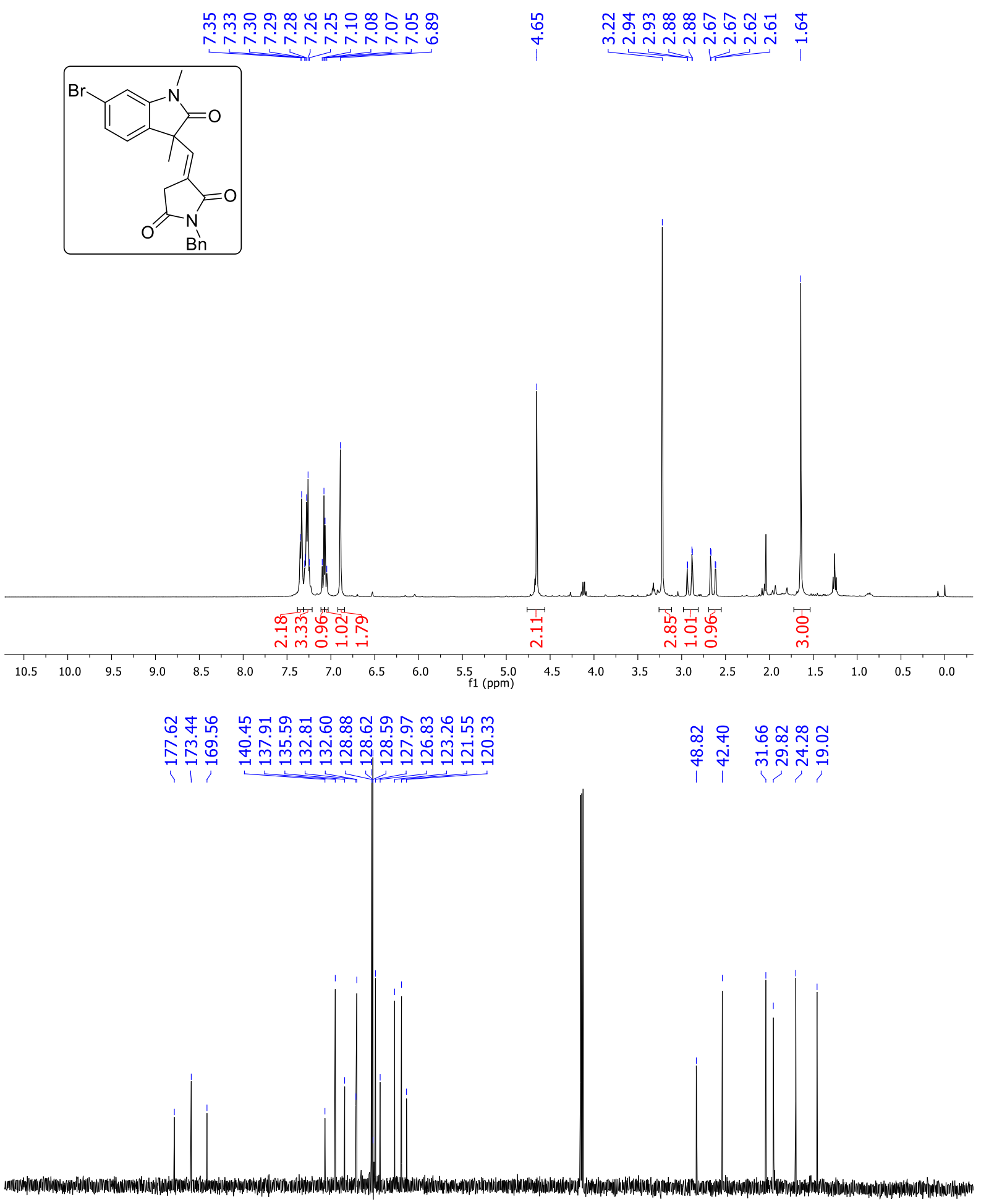

$\begin{array}{lllllllllllllllllllllllll}210 & 200 & 190 & 180 & 170 & 160 & 150 & 140 & 130 & 120 & 110 & \begin{array}{c}100 \\ \mathrm{f} 1(\mathrm{ppm})\end{array} & 90 & 80 & 70 & 60 & 50 & 40 & 30 & 20 & 10 & 0 & -10 & \end{array}$ 


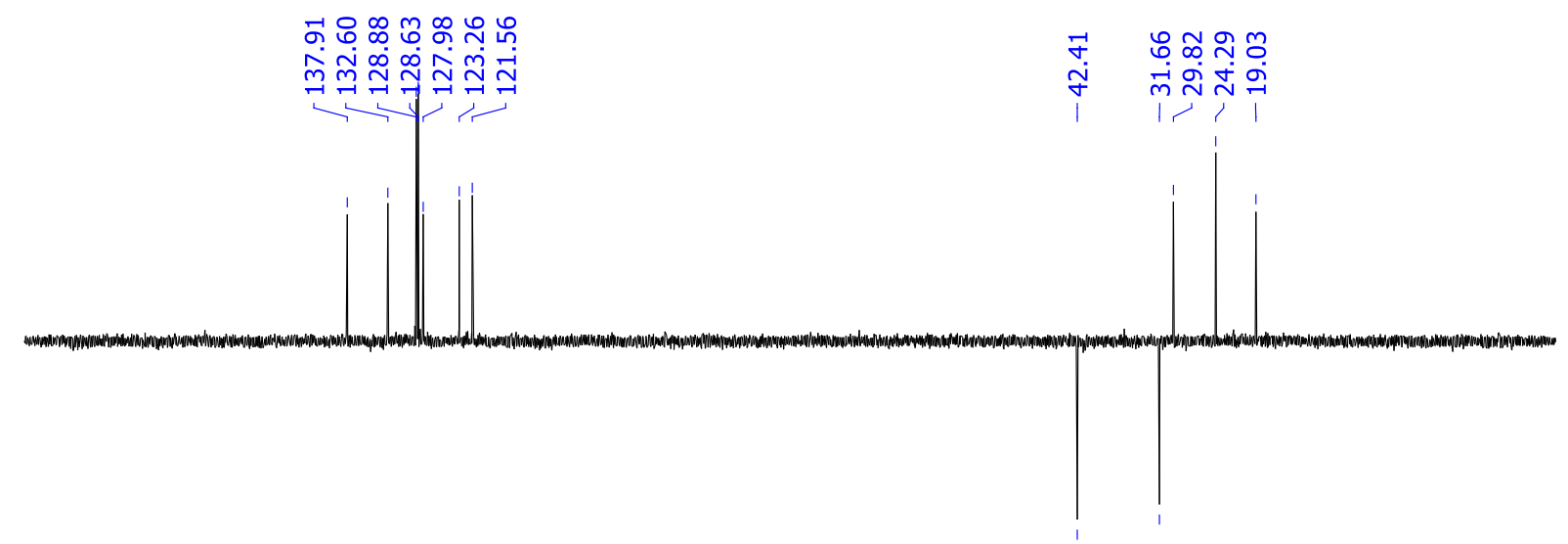

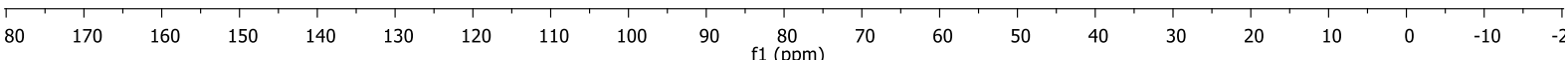


Copies of $\mathrm{H}^{1}, \mathrm{C}^{13}$ and DEPT 135 NMR spectra of compound 3ra'

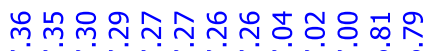

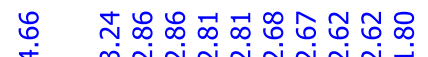

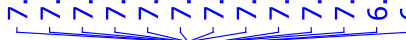
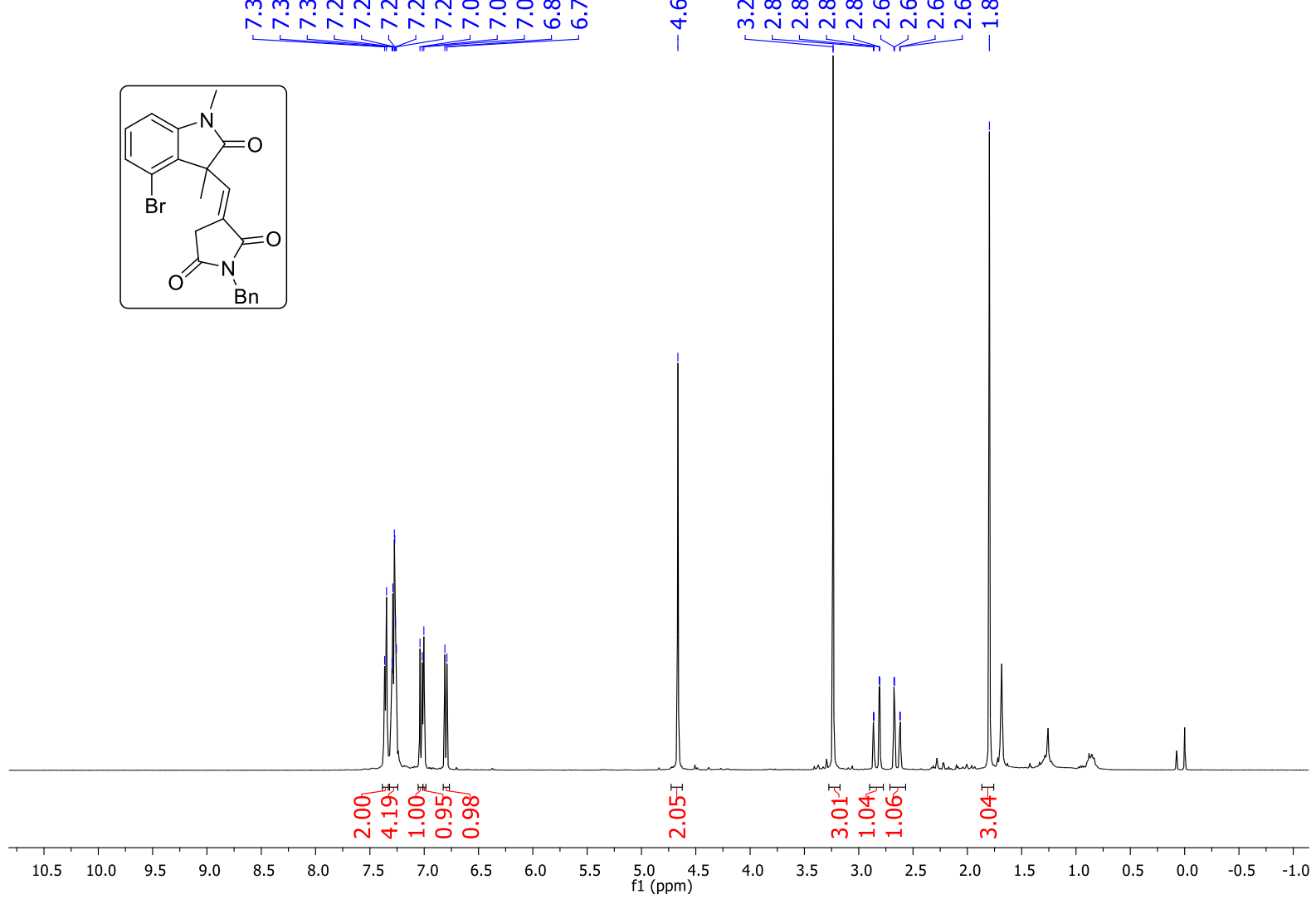

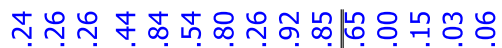

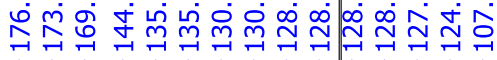

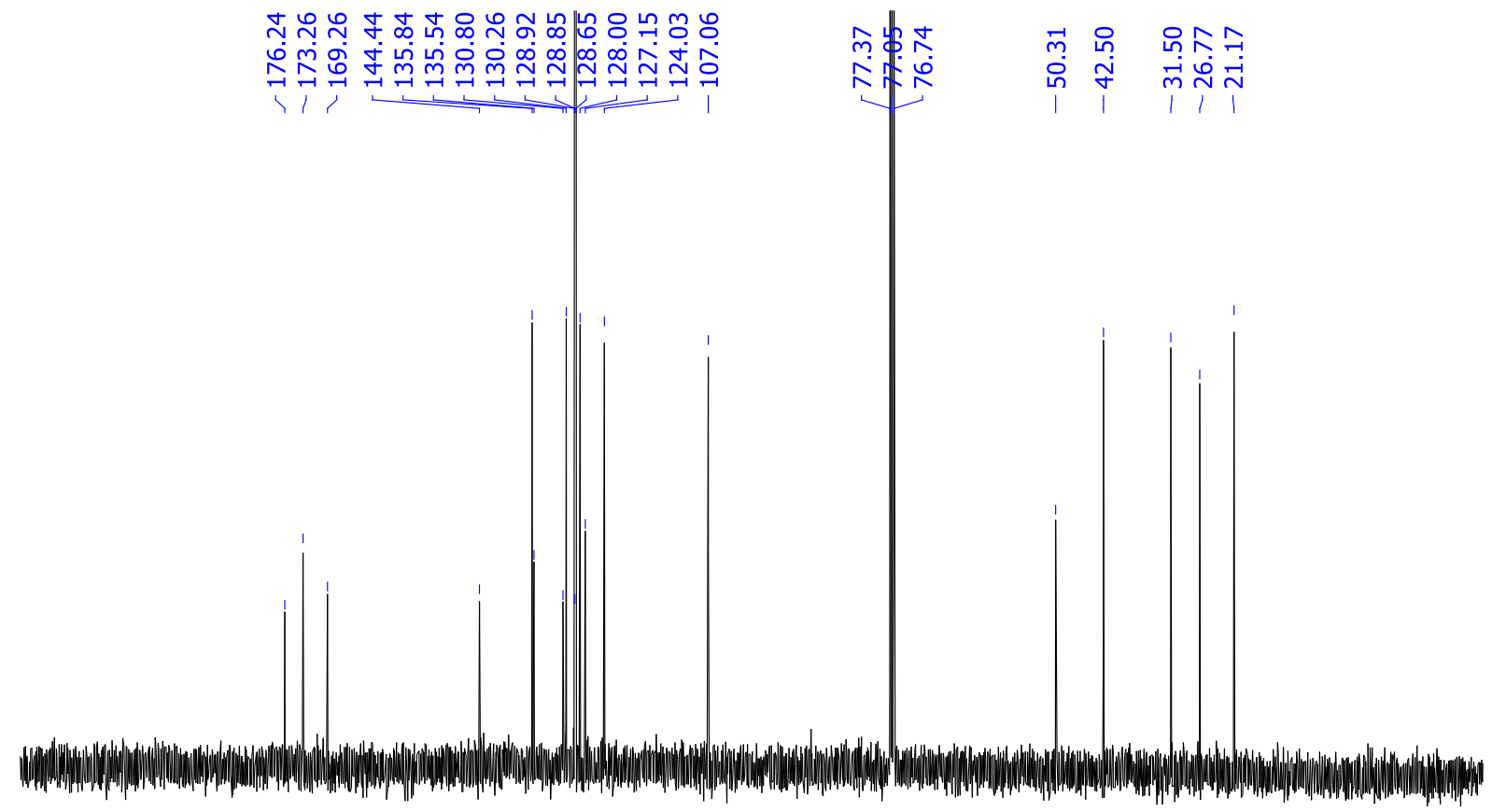

$\begin{array}{lllllllllll}210 & 200 & 190 & 180 & 170 & 160 & 150 & 140 & 130 & 120 & 110 \\ \mathrm{f} 1(\mathrm{ppm}) & 100\end{array}$ 


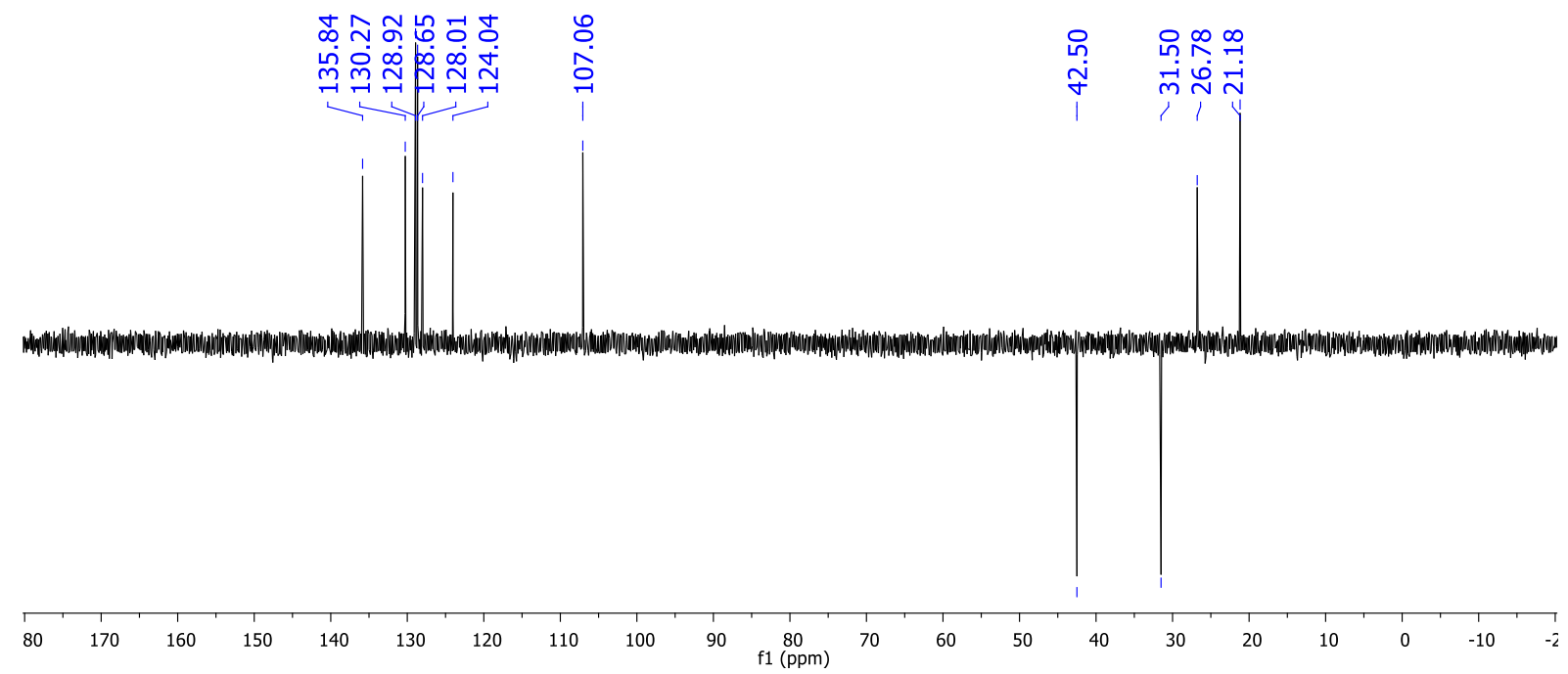


Copies of $\mathrm{H}^{1}, \mathrm{C}^{13}$ and DEPT 135 NMR spectra of compound 3sa

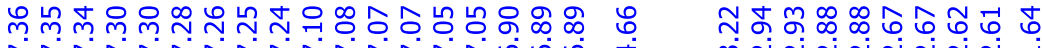

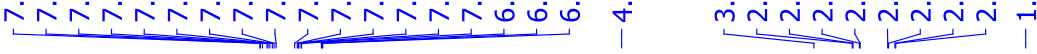
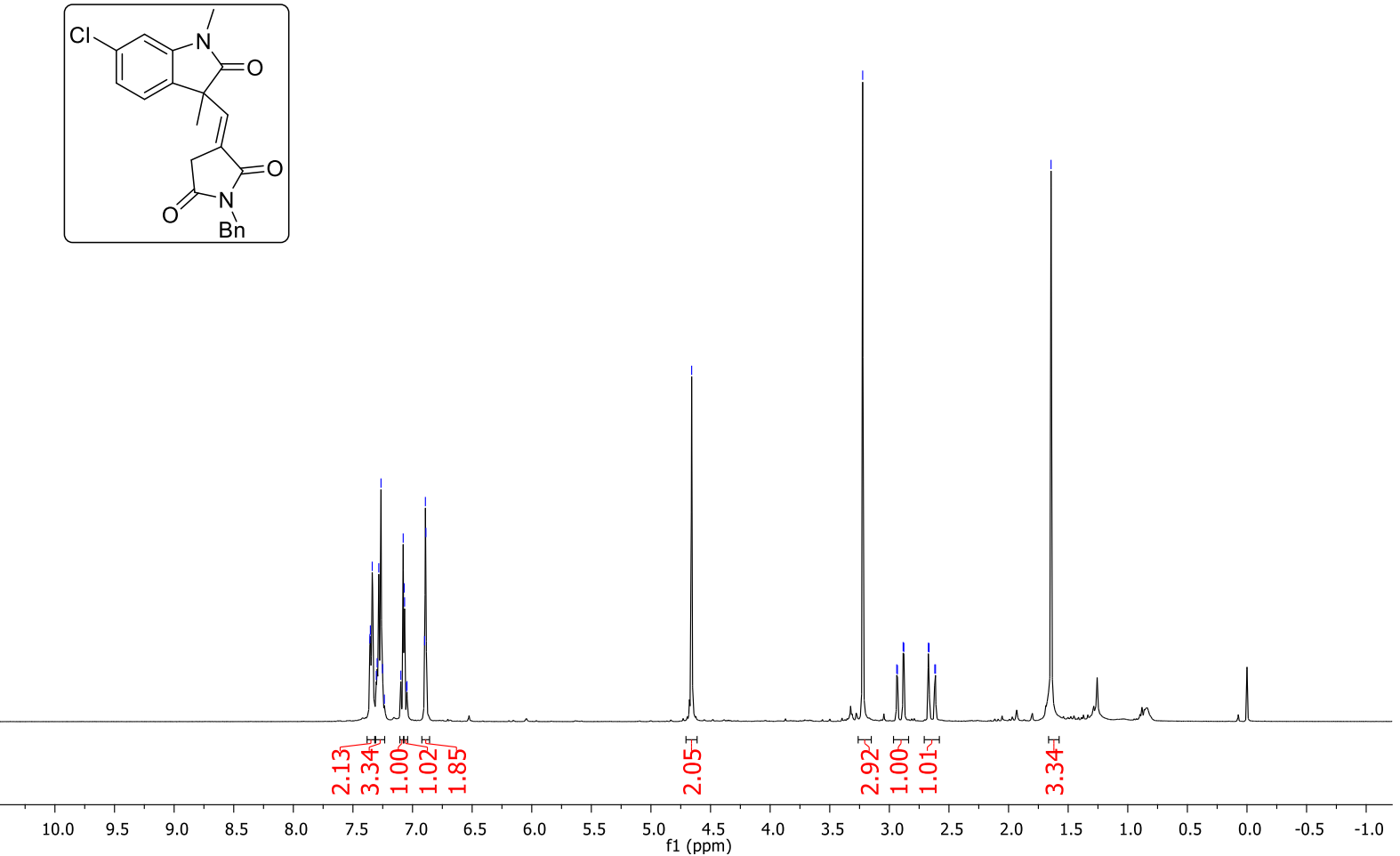

ळ

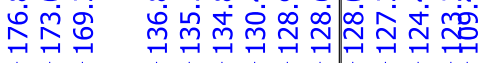

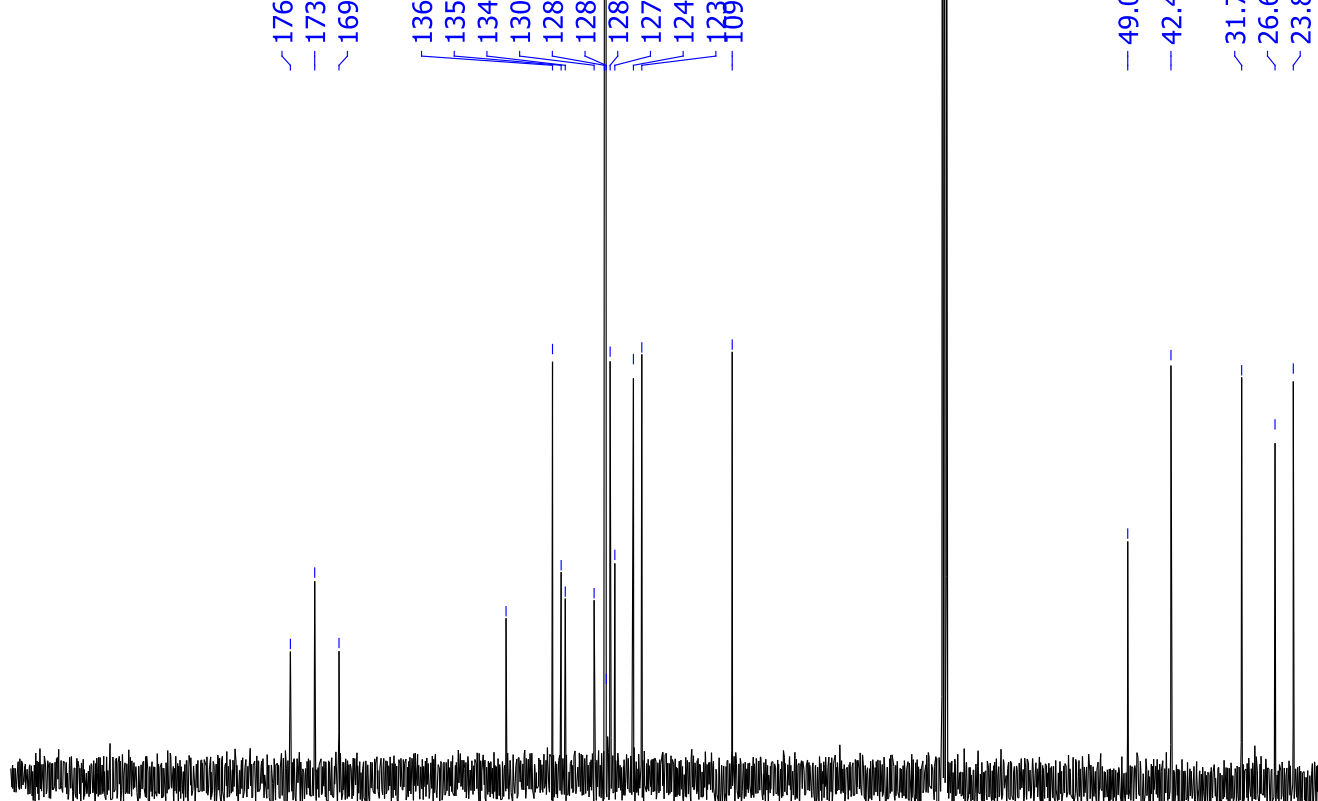

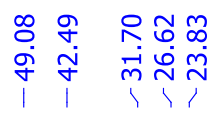

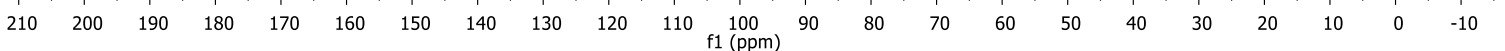




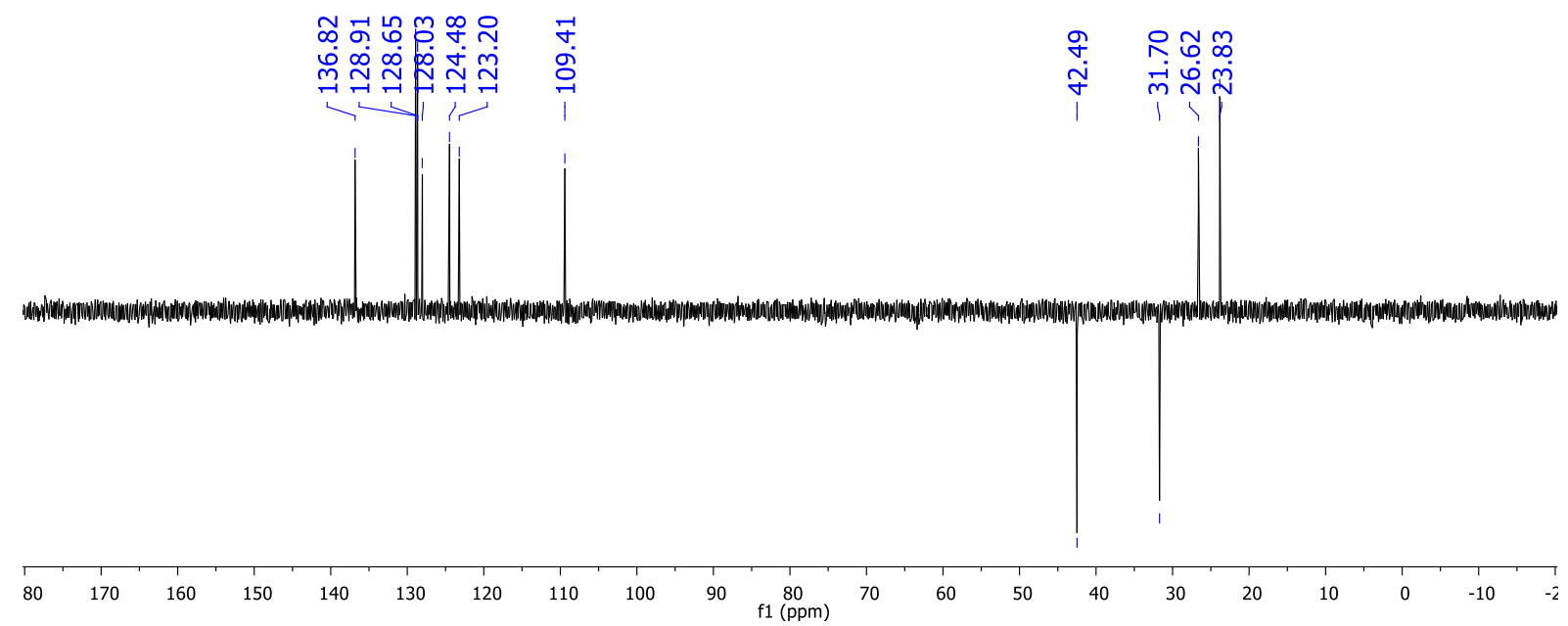


Copies of $\mathrm{H}^{1}, \mathrm{C}^{13}$ and DEPT 135 NMR spectra of compound 3sa'

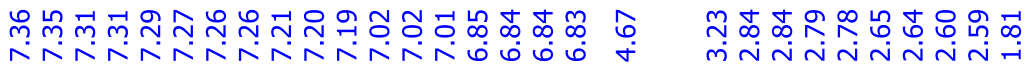

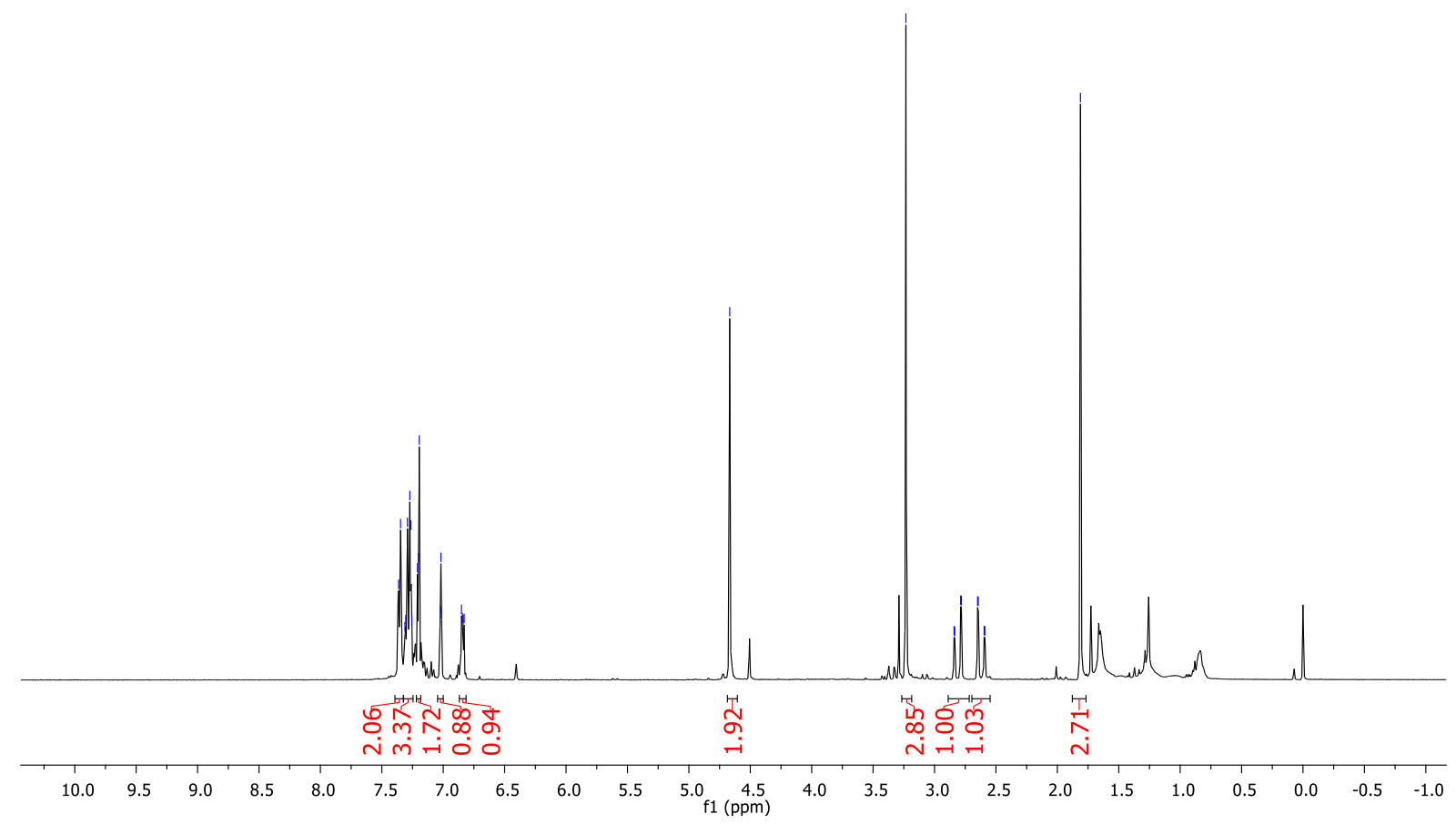

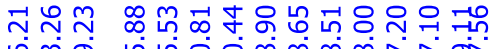

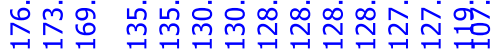

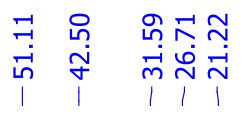

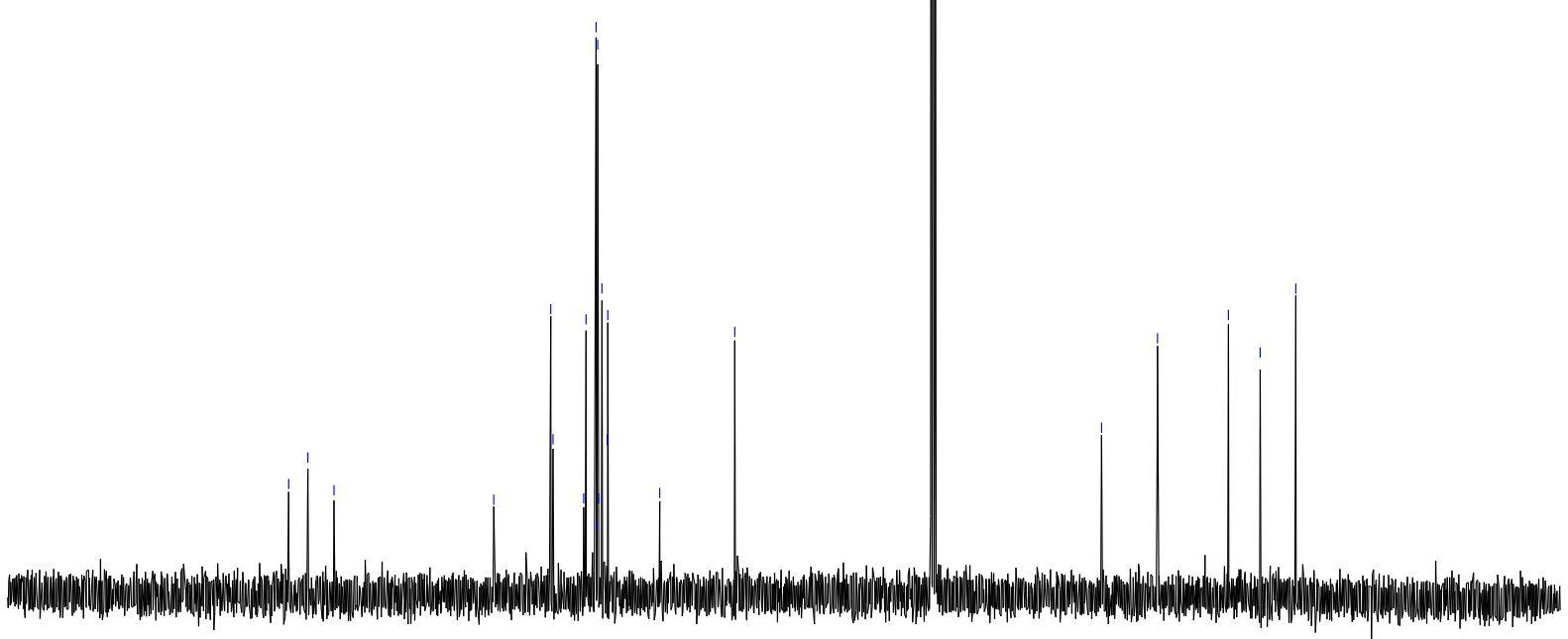

$\begin{array}{llllllllllllllllllllllll}210 & 200 & 190 & 180 & 170 & 160 & 150 & 140 & 130 & 120 & 110 & \underset{\mathrm{f} 1}{100}(\mathrm{ppm}) & 90 & 80 & 70 & 60 & 50 & 40 & 30 & 20 & 10 & 0 & -10\end{array}$ 


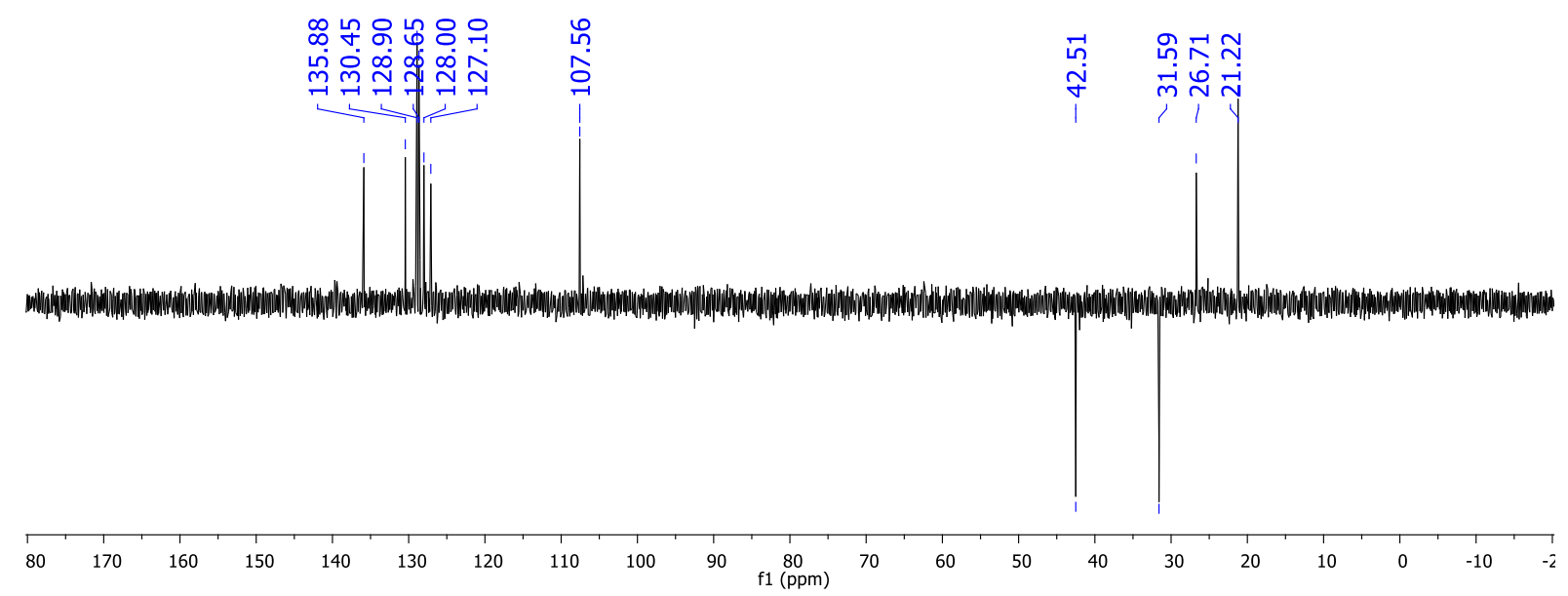


Copies of $\mathrm{H}^{1}, \mathrm{C}^{13}$ and DEPT 135 NMR spectra of compound 3ta

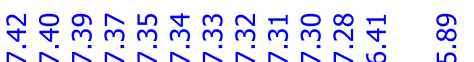

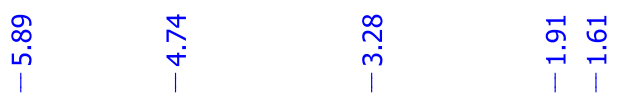<smiles></smiles>

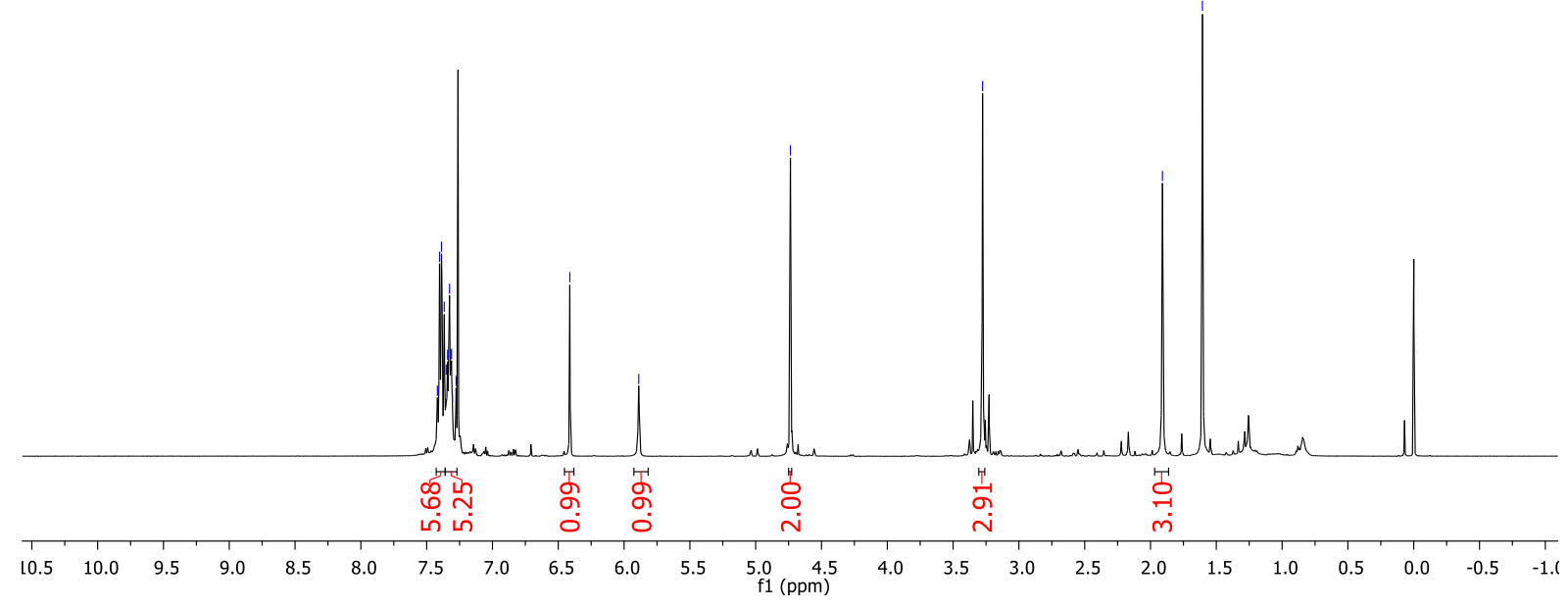

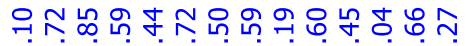

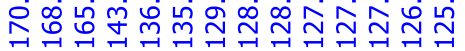

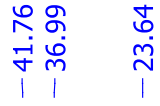

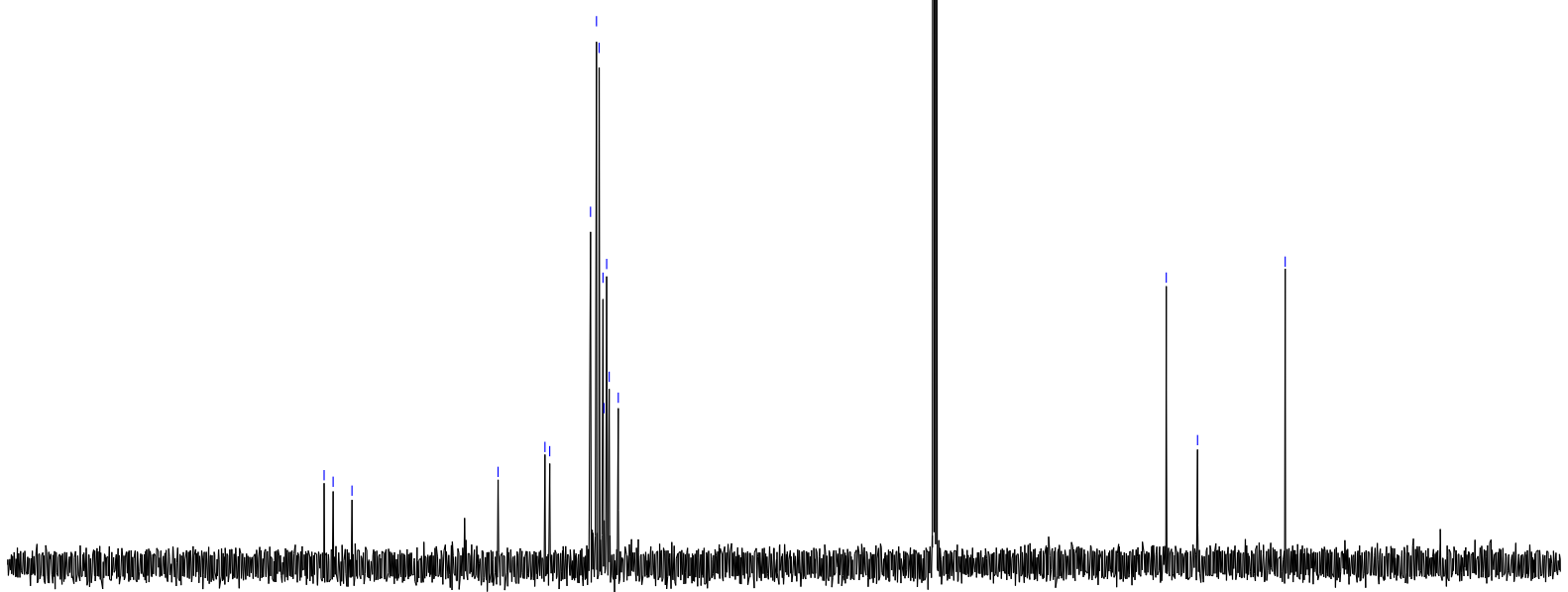

$\begin{array}{lllllllllllllllllllllllll}210 & 200 & 190 & 180 & 170 & 160 & 150 & 140 & 130 & 120 & 110 & \begin{array}{c}100 \\ \mathrm{f} 1(\mathrm{ppm})\end{array} & 90 & 80 & 70 & 60 & 50 & 40 & 30 & 20 & 10 & 0 & -10\end{array}$ 


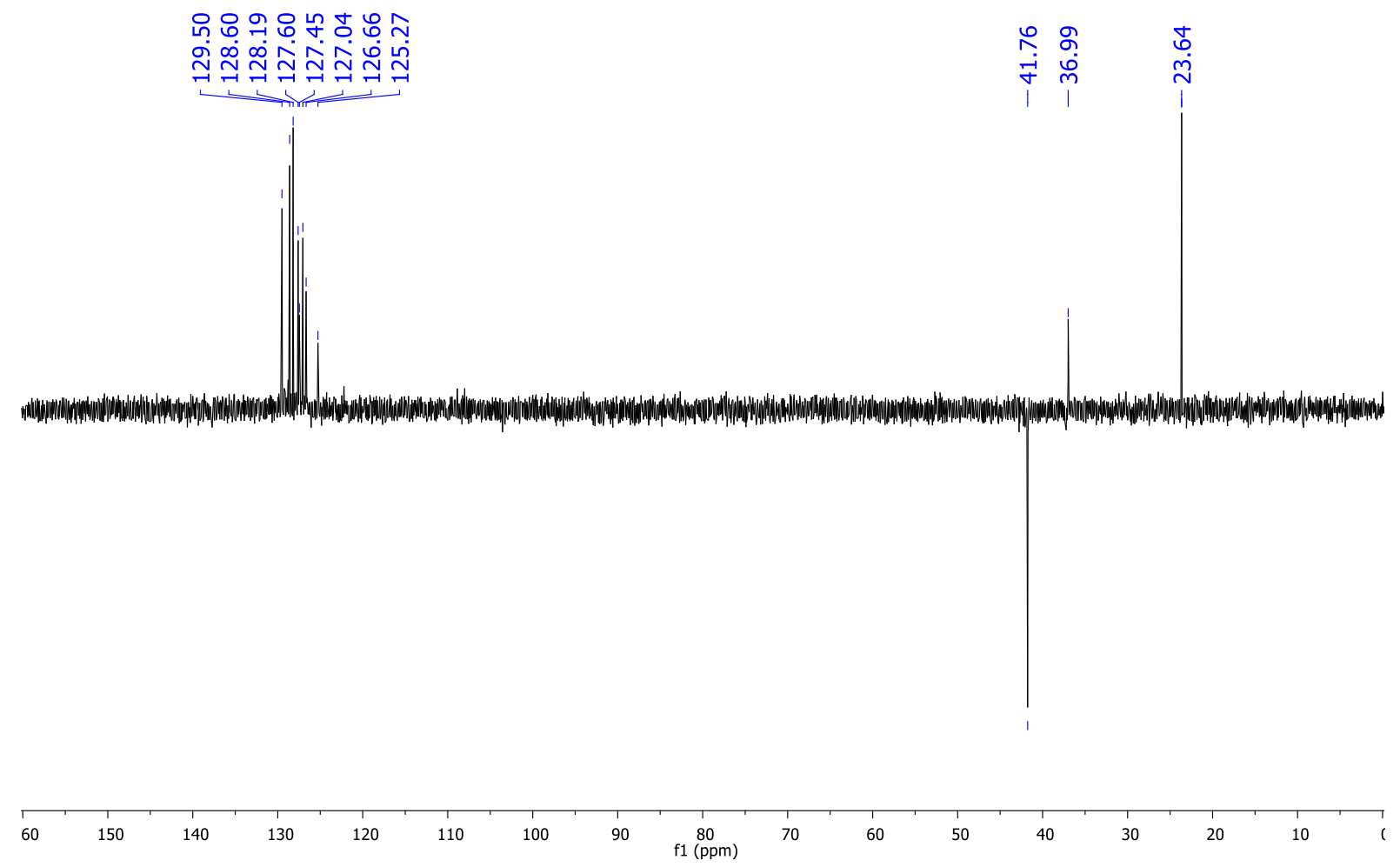


Copies of $\mathrm{H}^{1}, \mathrm{C}^{13}$ and DEPT 135 NMR spectra of compound 3ta' ㄱ.

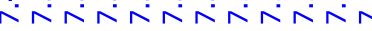

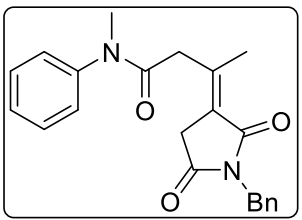

$\mathrm{Bn}$

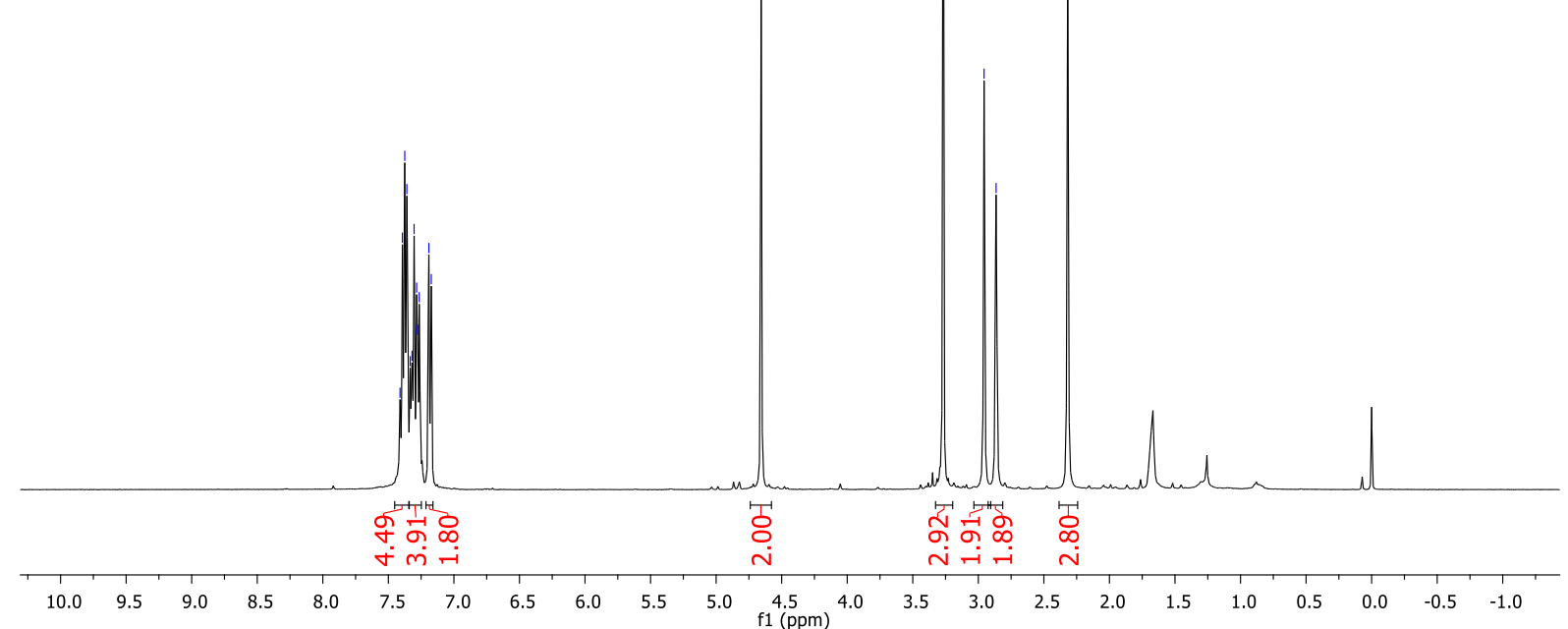

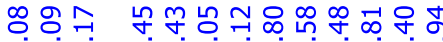

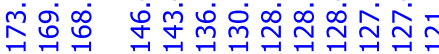

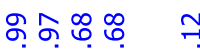

भै

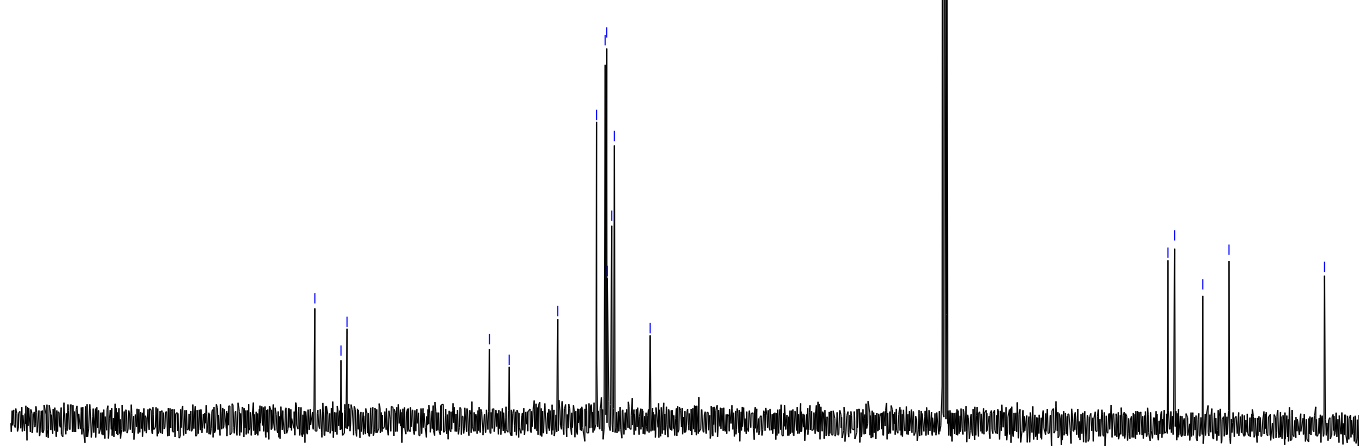

$\begin{array}{lllllllllllllllllllllll}210 & 200 & 190 & 180 & 170 & 160 & 150 & 140 & 130 & 120 & 110 & \begin{array}{c}100 \\ \mathrm{f} 1(\mathrm{ppm})\end{array} & 90 & 80 & 70 & 60 & 50 & 40 & 30 & 20 & 10 & 0 & -10\end{array}$ 


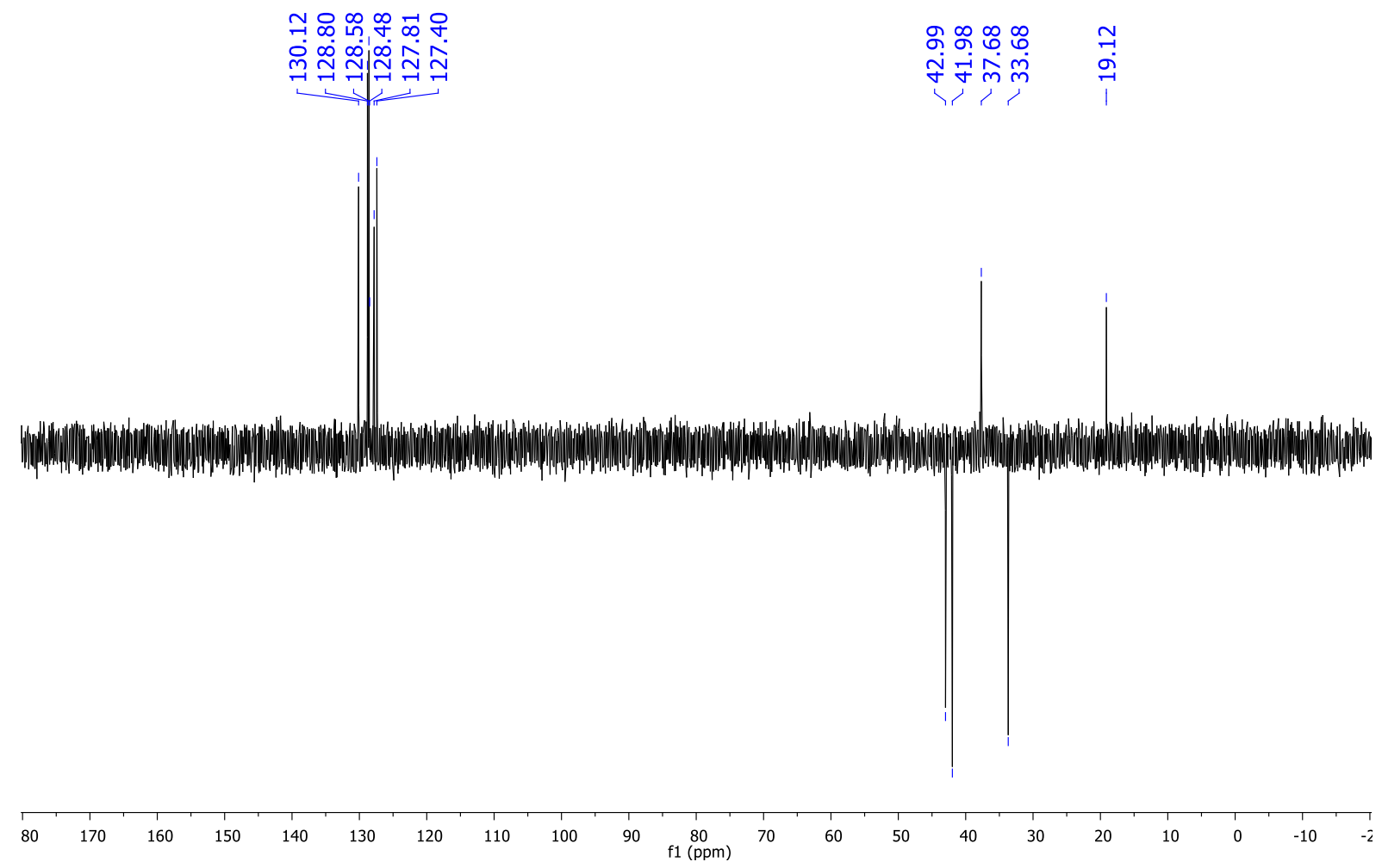

Copy of NOESY Experiment of Compound 3ta'

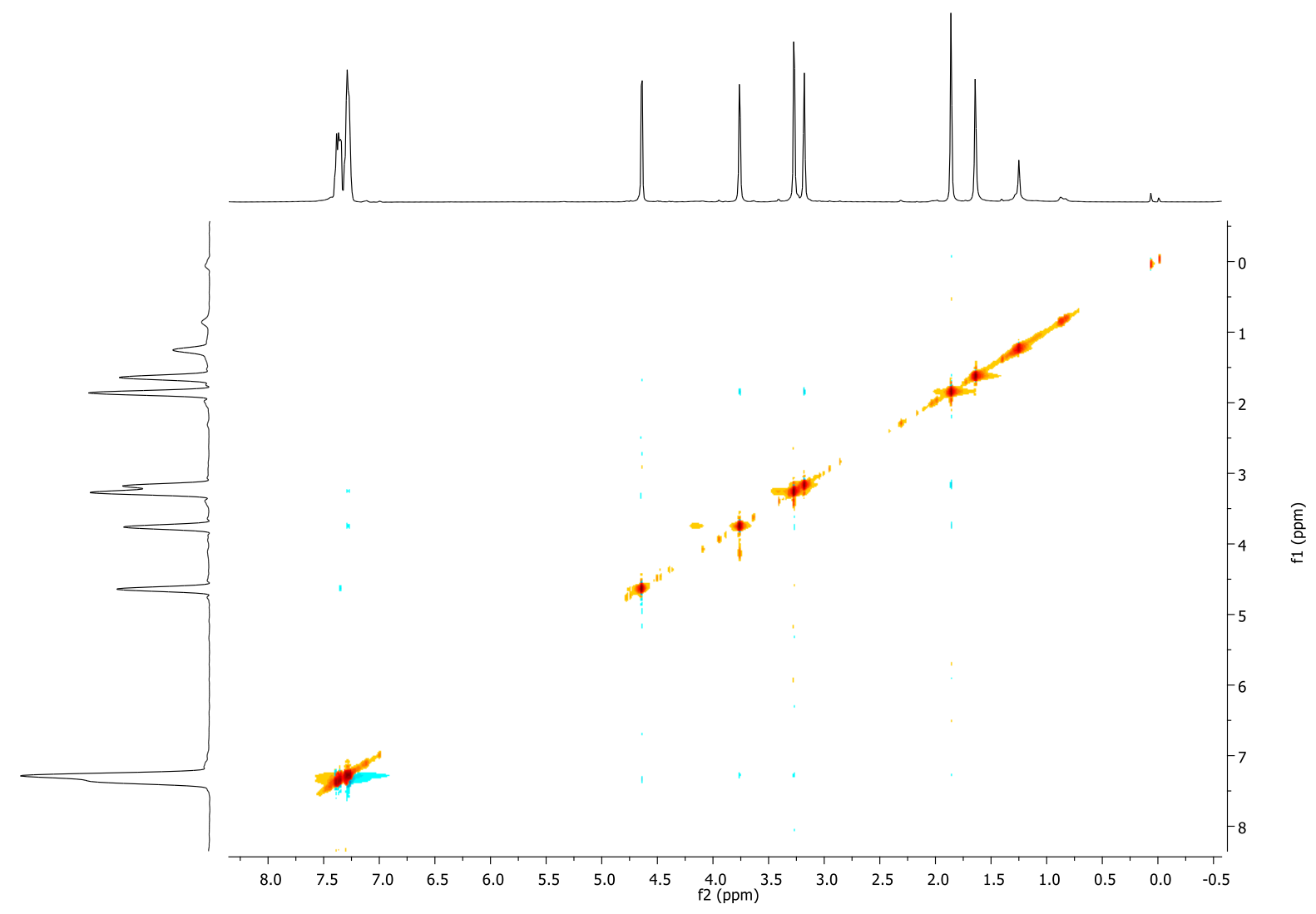


Copies of $\mathrm{H}^{1}, \mathrm{C}^{13}$ and DEPT 135 NMR spectra of compound 3ta"'

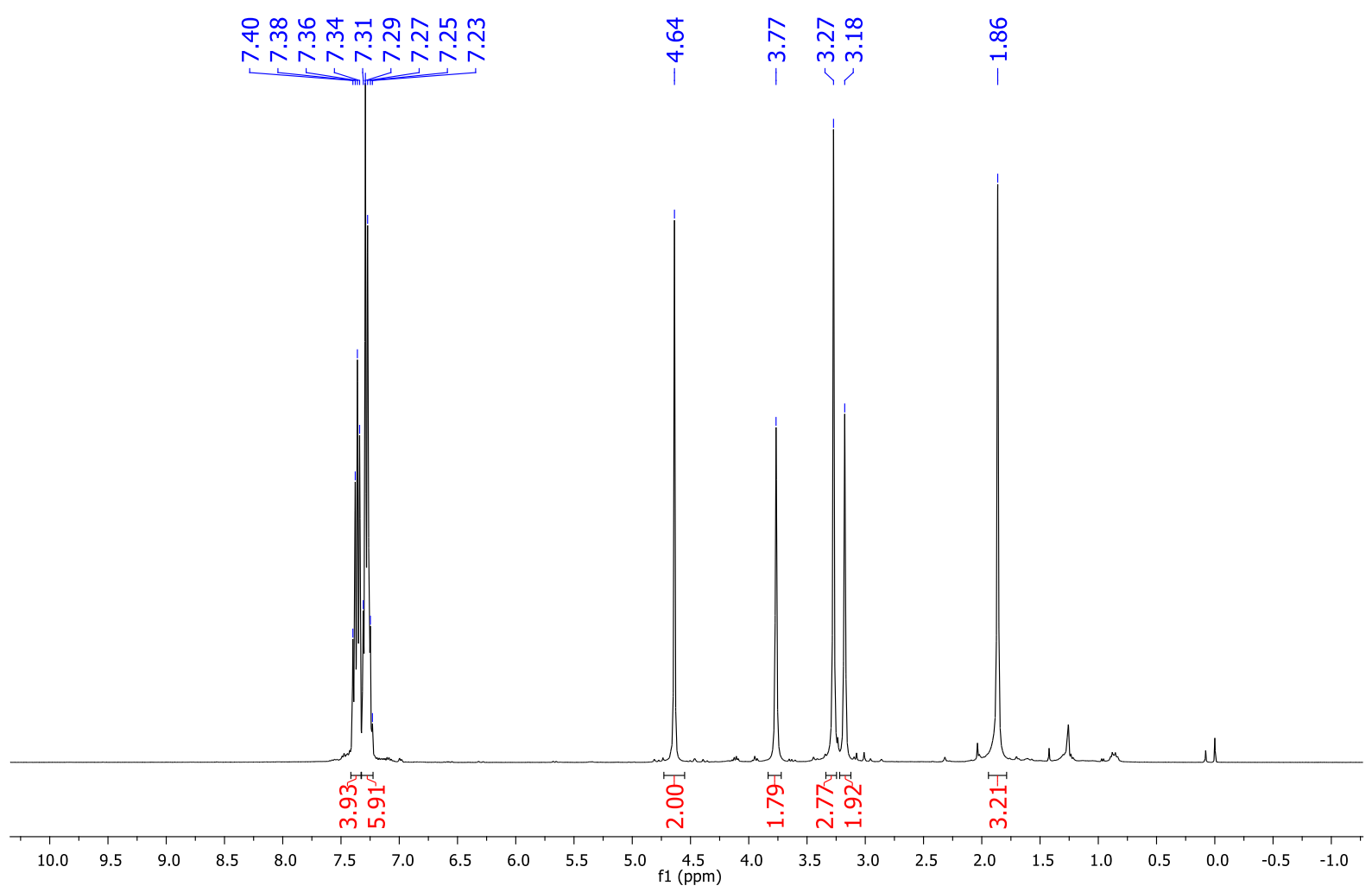

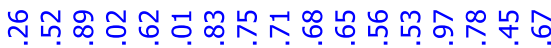

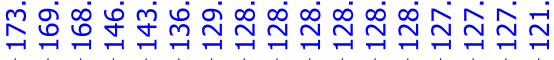

ตูํํำ 눙

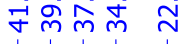

iा

।? ।

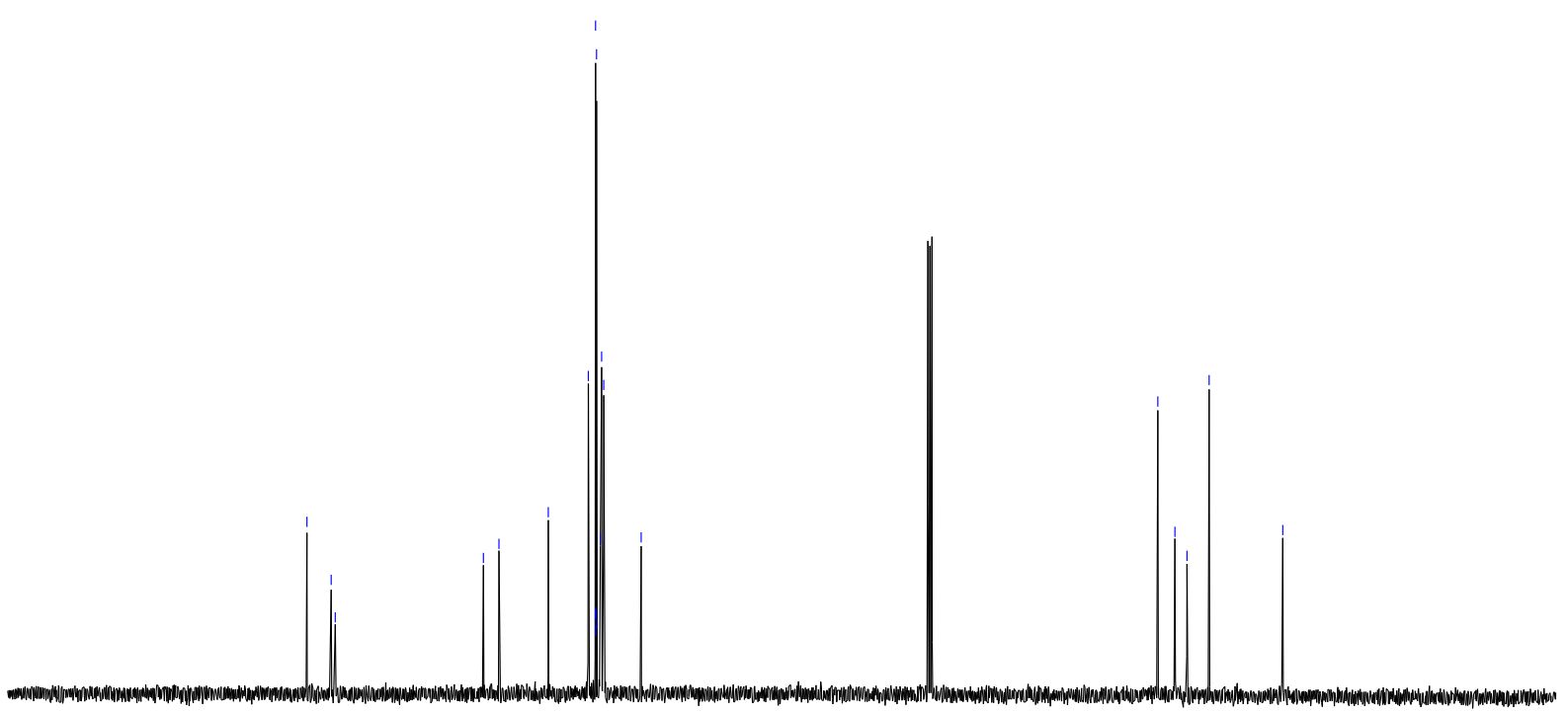

$\begin{array}{lllllllllllllllllllllll}210 & 200 & 190 & 180 & 170 & 160 & 150 & 140 & 130 & 120 & 110 & \underset{\mathrm{f} 1(\mathrm{ppm})}{100} & 90 & 80 & 70 & 60 & 50 & 40 & 30 & 20 & 10 & 0 & -10\end{array}$ 


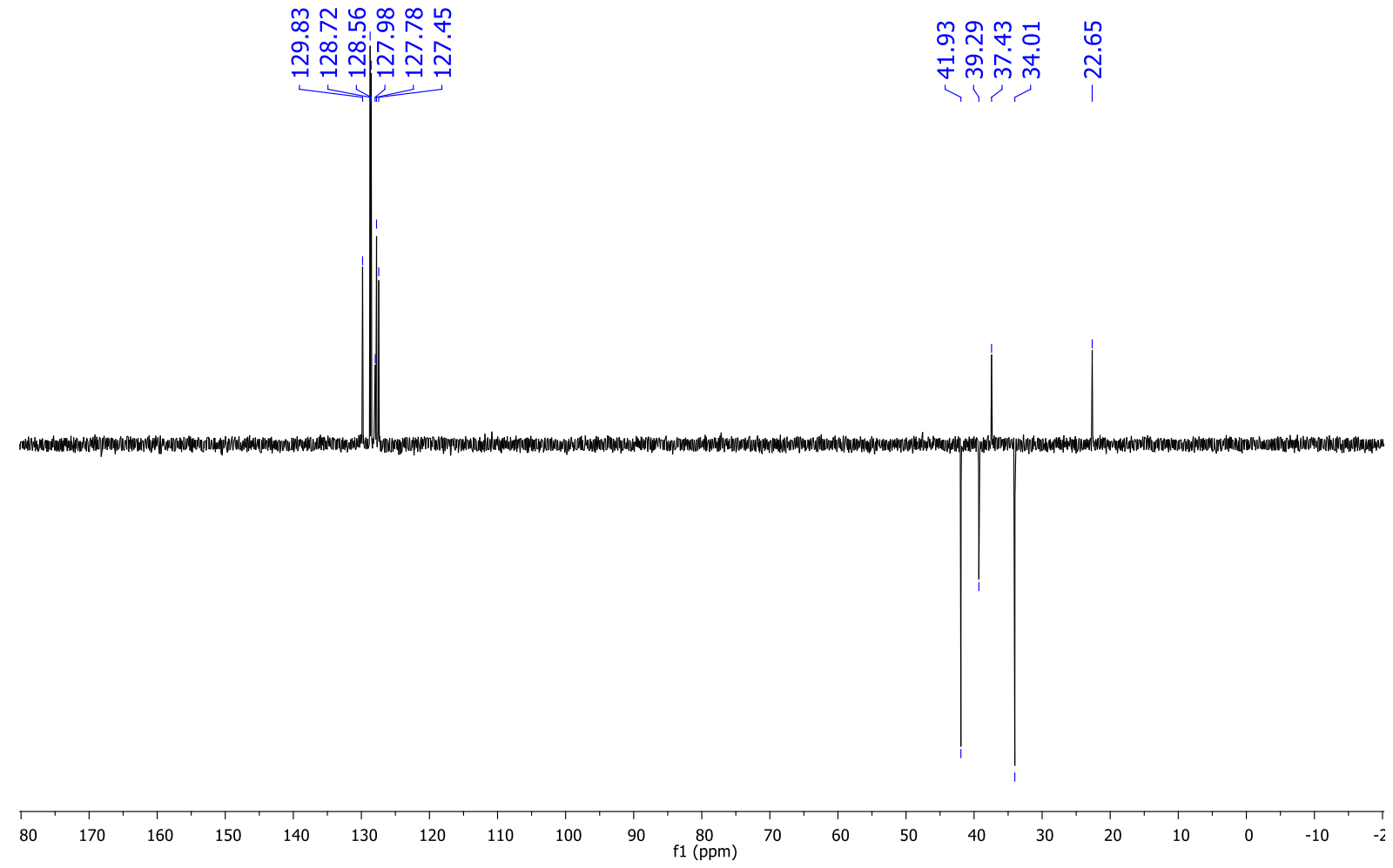

Copy of NOESY Experiment of Compound 3ta'

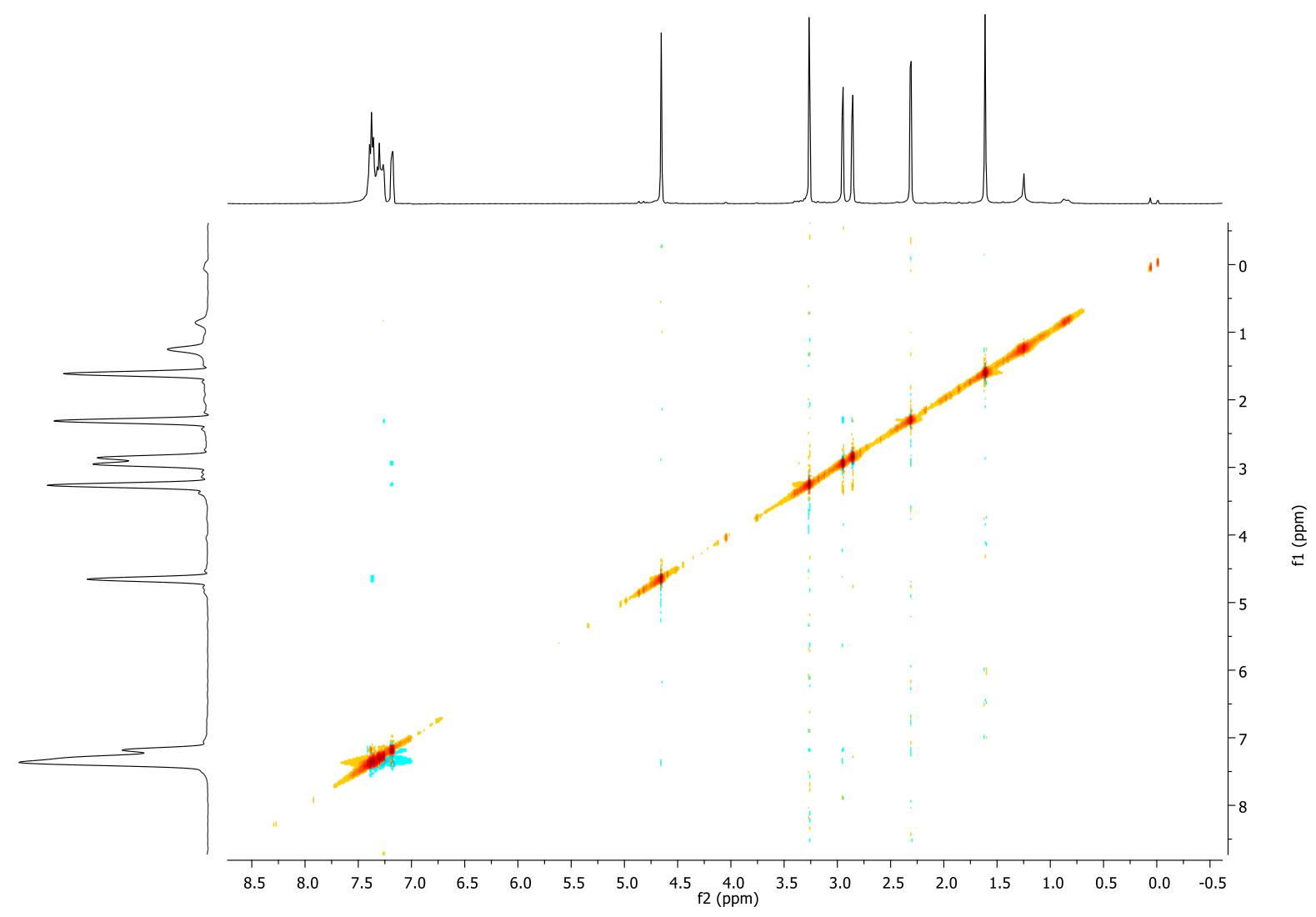


Copies of $\mathrm{H}^{1}, \mathrm{C}^{13}$ and DEPT 135 NMR spectra of compound 3ab

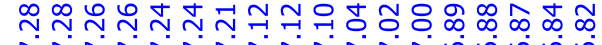

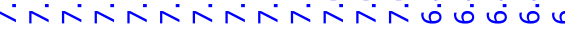

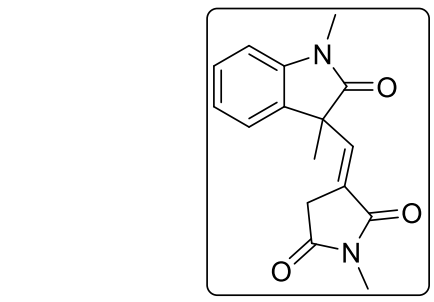

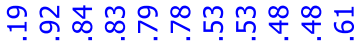
minjusininin
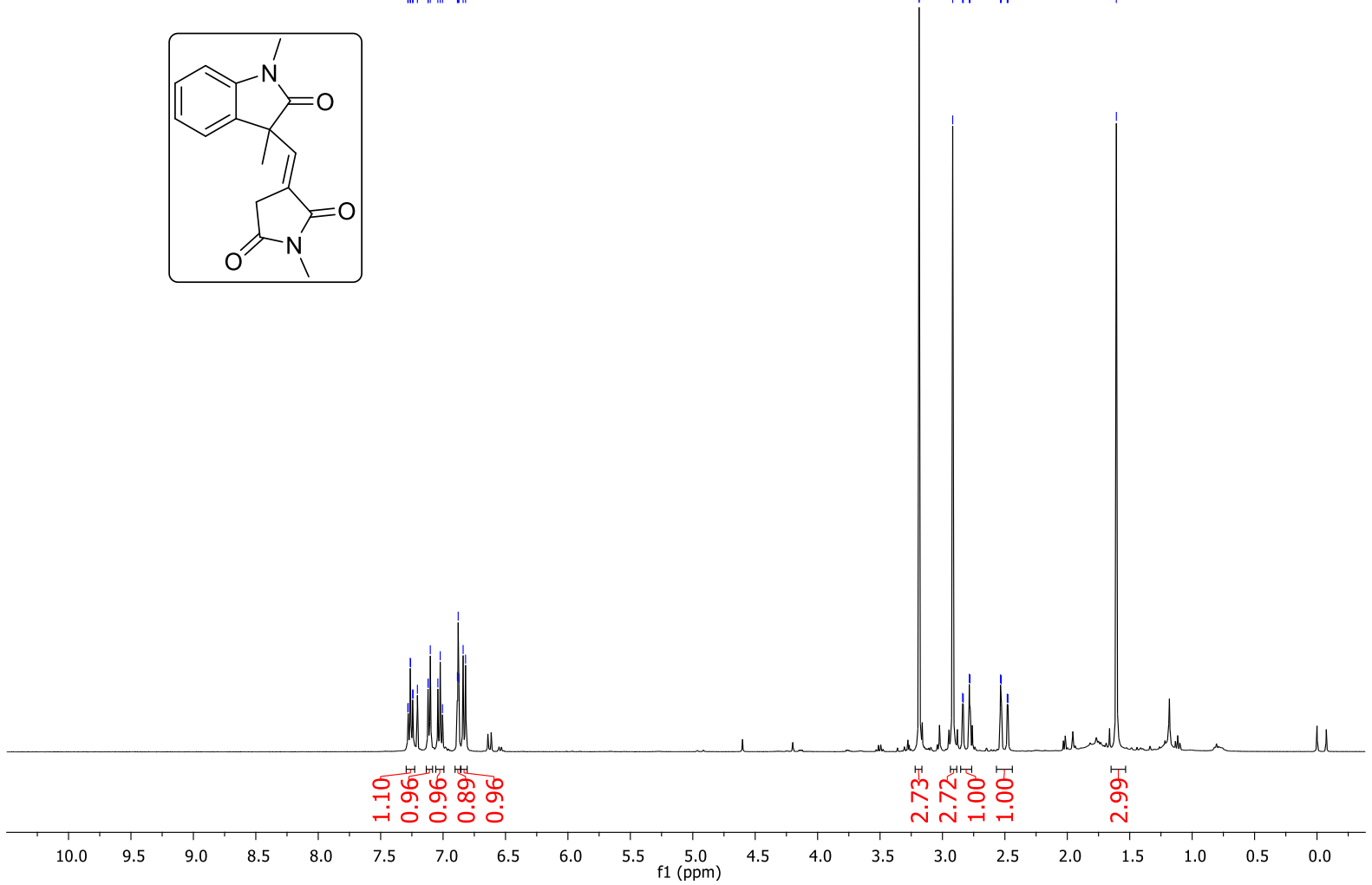

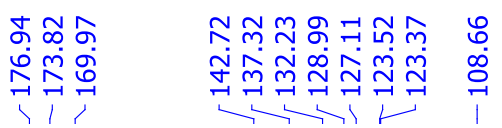

m.

l.

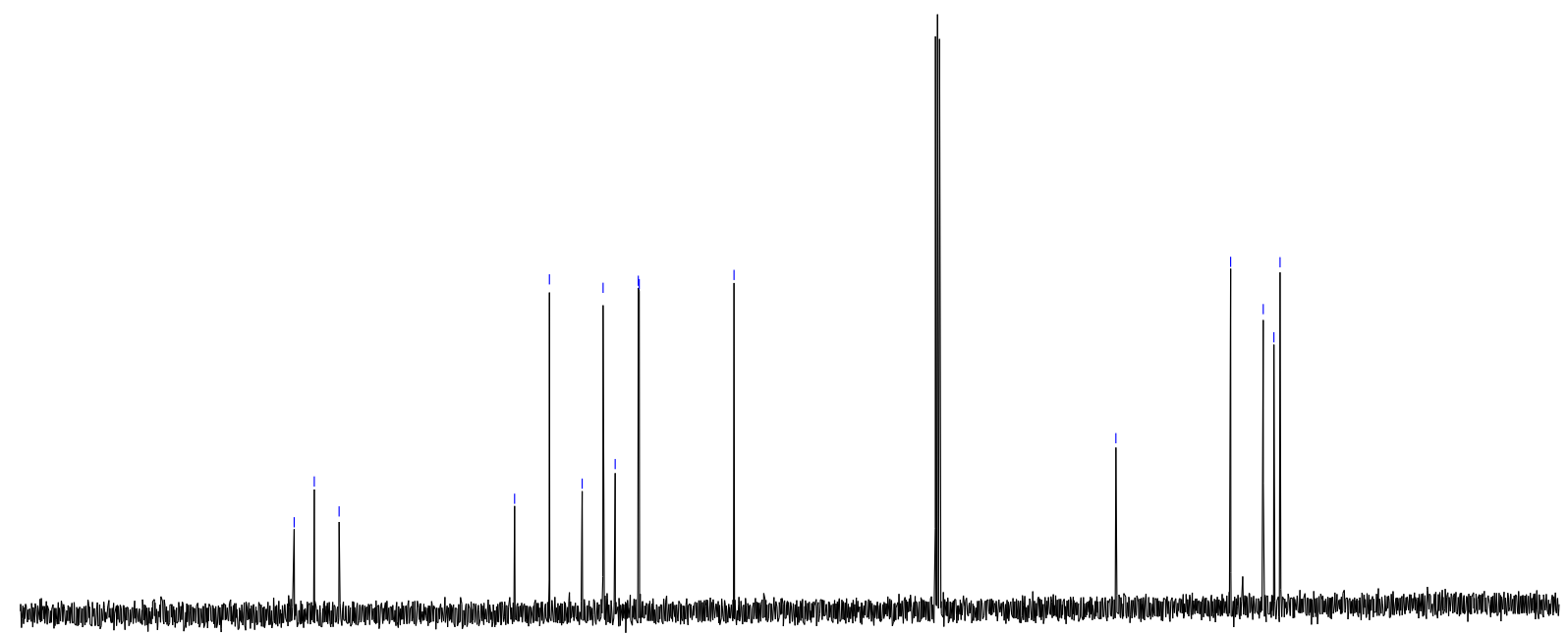

$\begin{array}{lllllllllllllllllllllll}210 & 200 & 190 & 180 & 170 & 160 & 150 & 140 & 130 & 120 & 110 & \begin{array}{c}100 \\ \mathrm{f} 1(\mathrm{ppm})\end{array} & 90 & 80 & 70 & 60 & 50 & 40 & 30 & 20 & 10 & 0 & -10\end{array}$ 


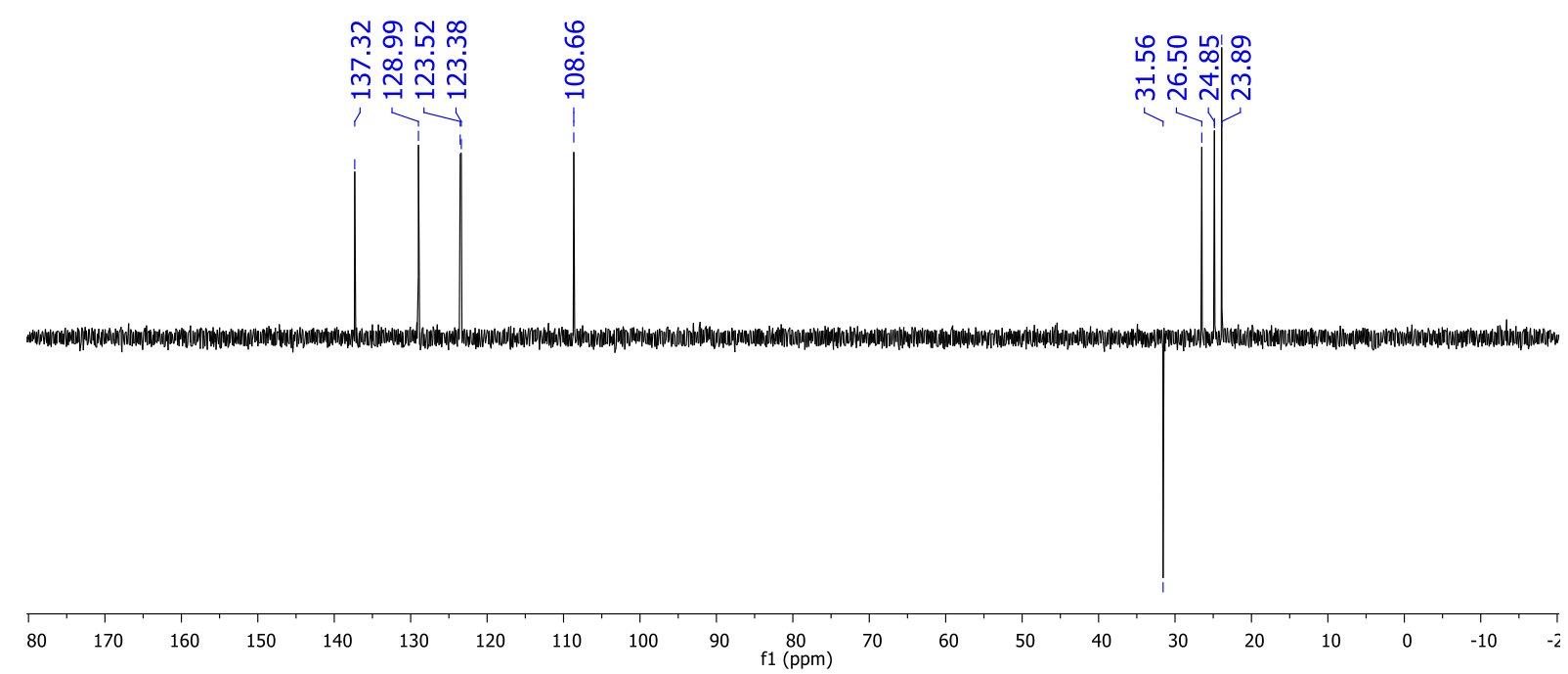


Copies of $\mathrm{H}^{1}, \mathrm{C}^{13}$ and DEPT 135 NMR spectra of compound 3ac

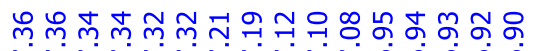

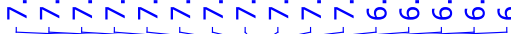

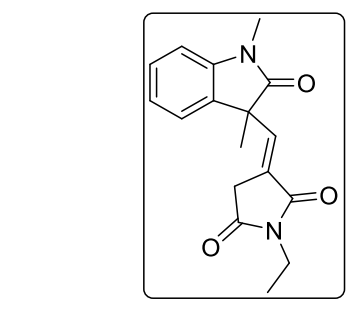

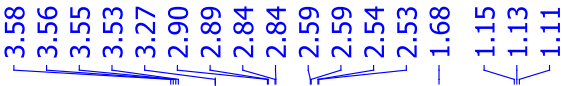
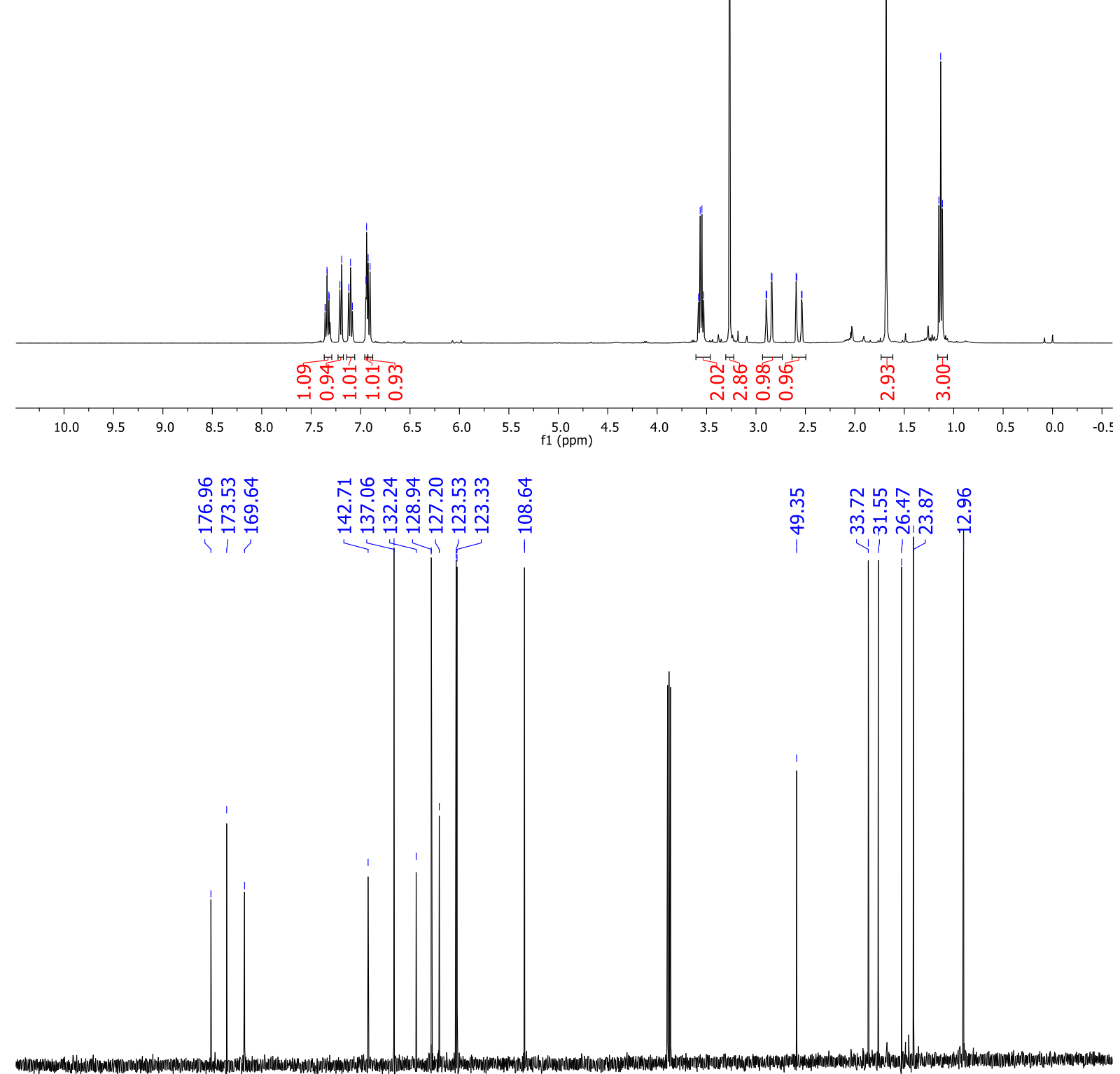

$\begin{array}{lllllllllllllllllllllllllllllll}210 & 200 & 190 & 180 & 170 & 160 & 150 & 140 & 130 & 120 & 110 & 100 & 90 & 80 & 70 & 60 & 50 & 40 & 30 & 20 & 10 & 0 & -10\end{array}$ 


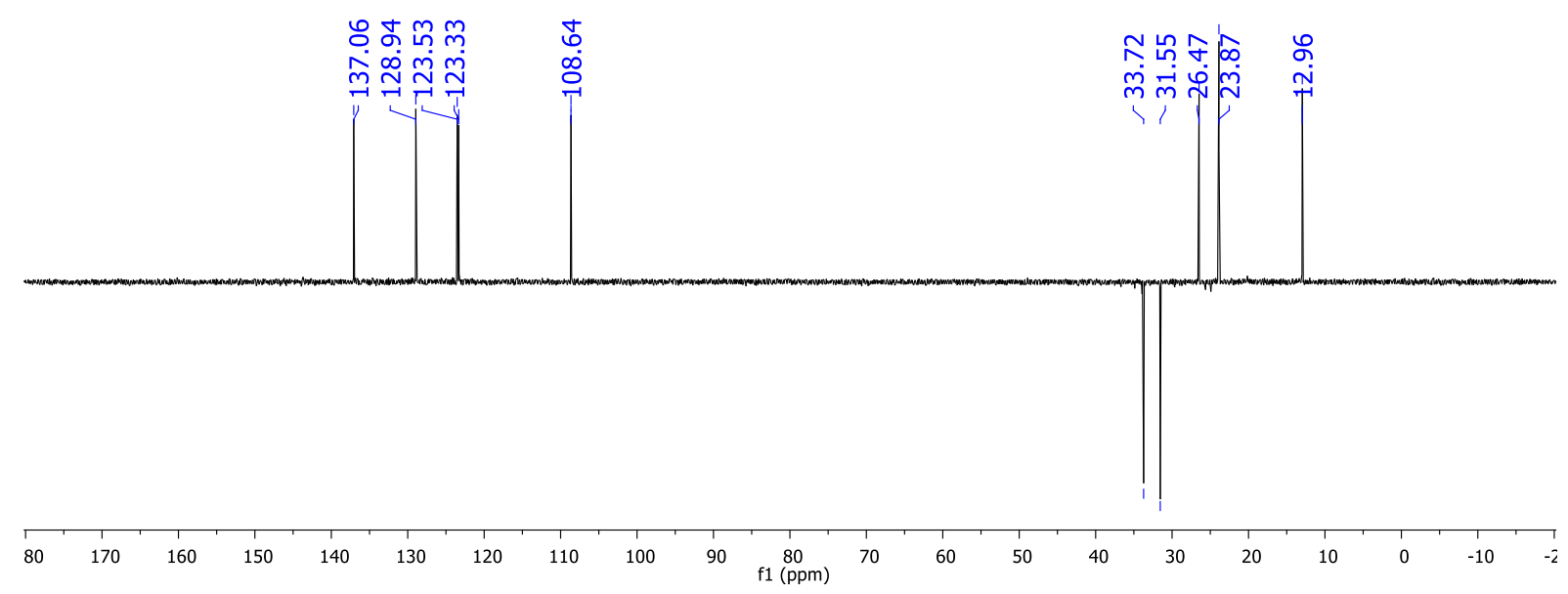


Copies of $\mathrm{H}^{1}, \mathrm{C}^{13}$ and DEPT 135 NMR spectra of compound 3ad

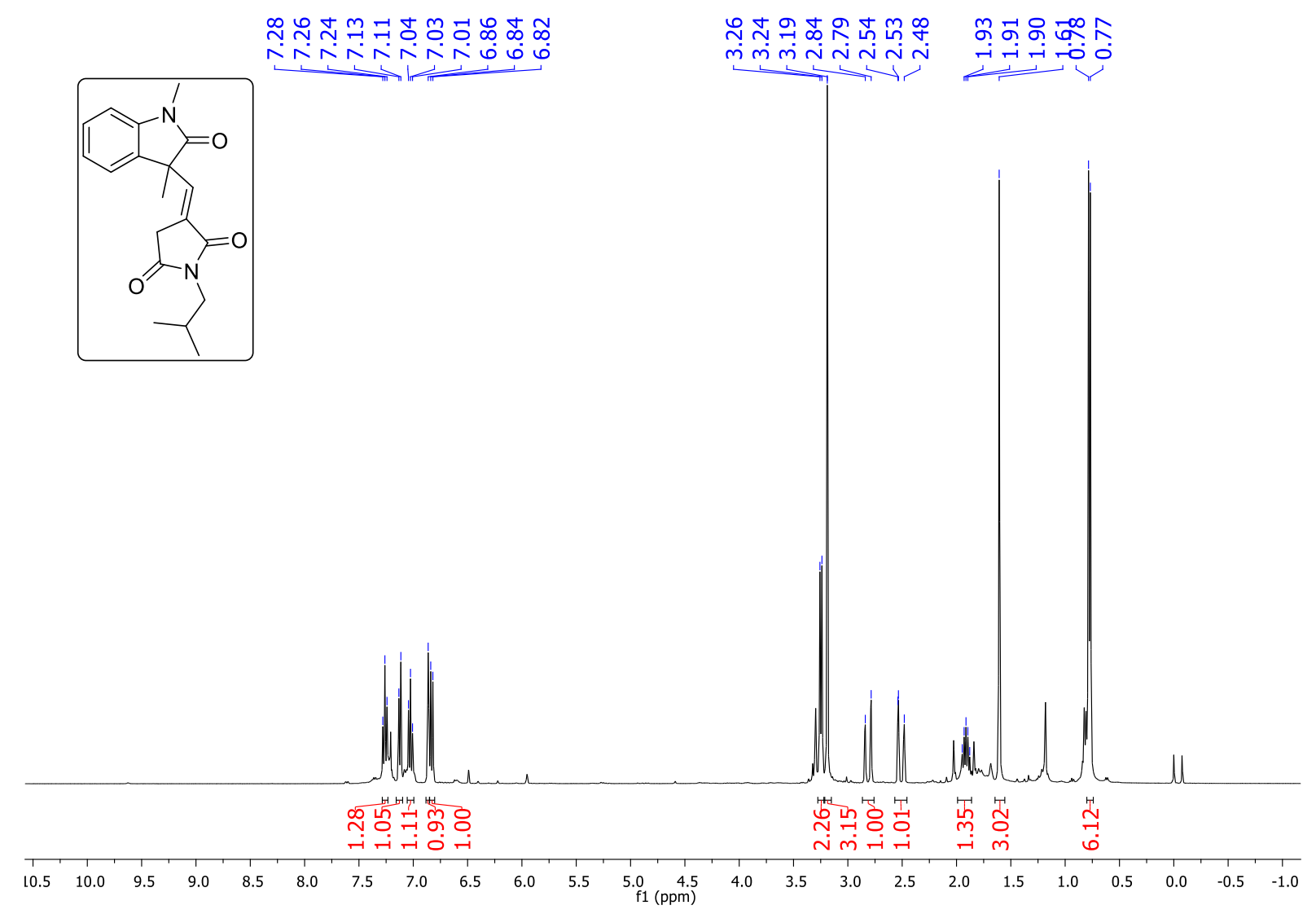

$$
\begin{aligned}
& \text { ํㅟㅁ f강ㅎㅇ }
\end{aligned}
$$

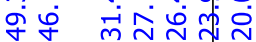

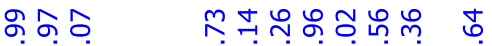

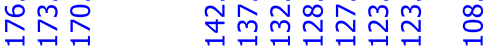

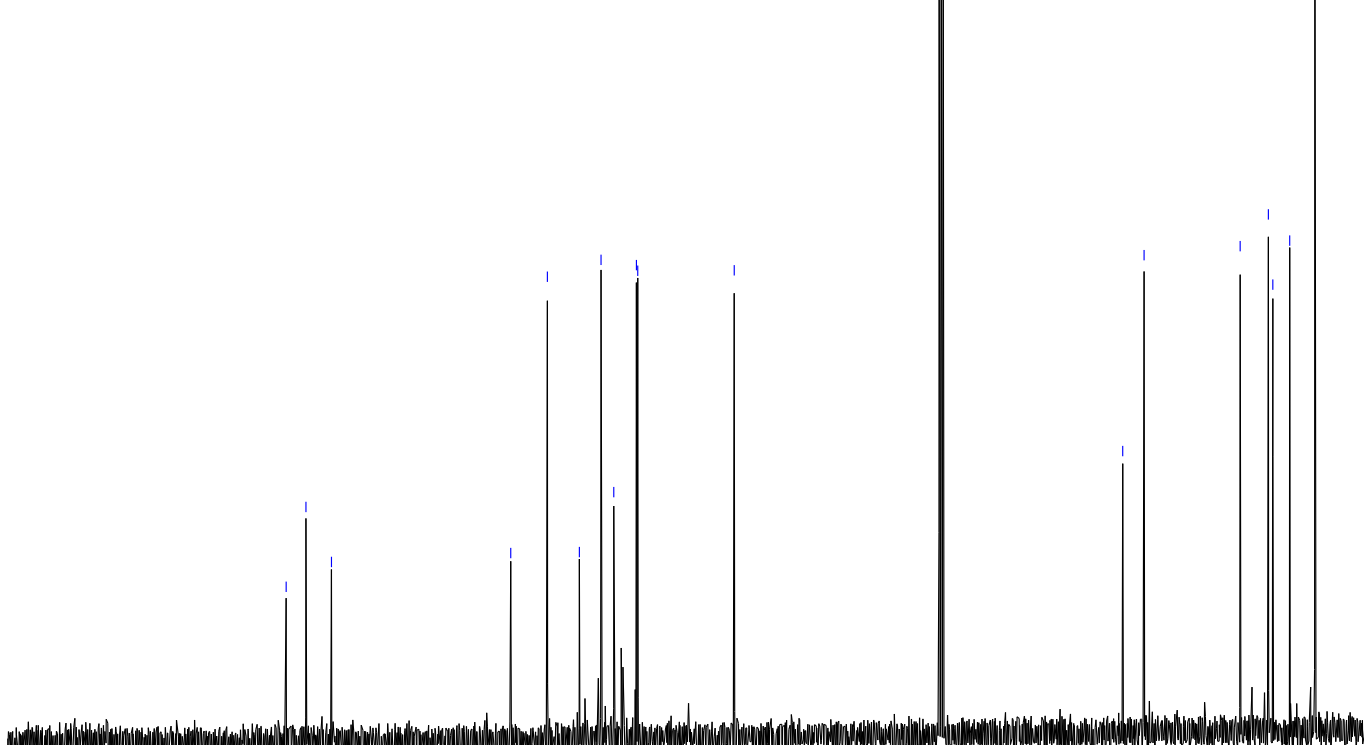

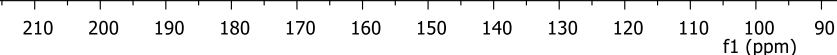




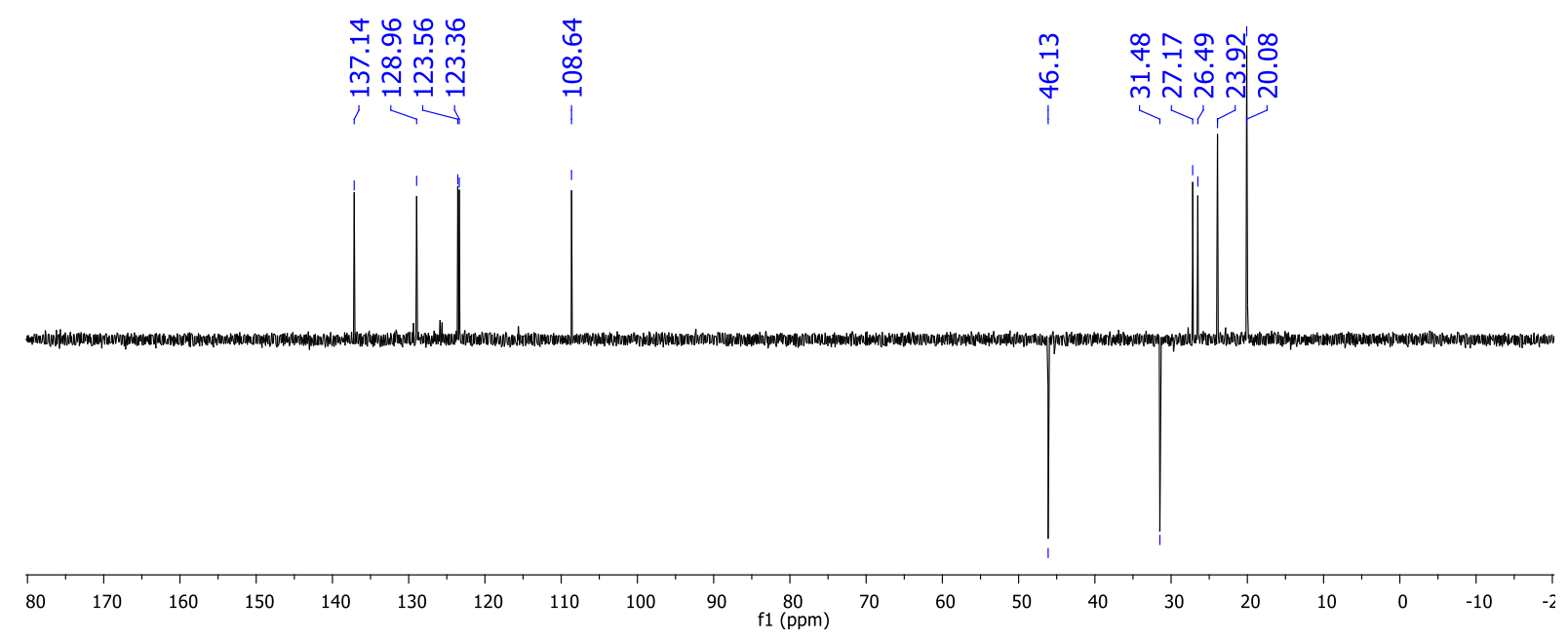


Copies of $\mathrm{H}^{1}, \mathrm{C}^{13}$ and DEPT 135 NMR spectra of compound 3ae

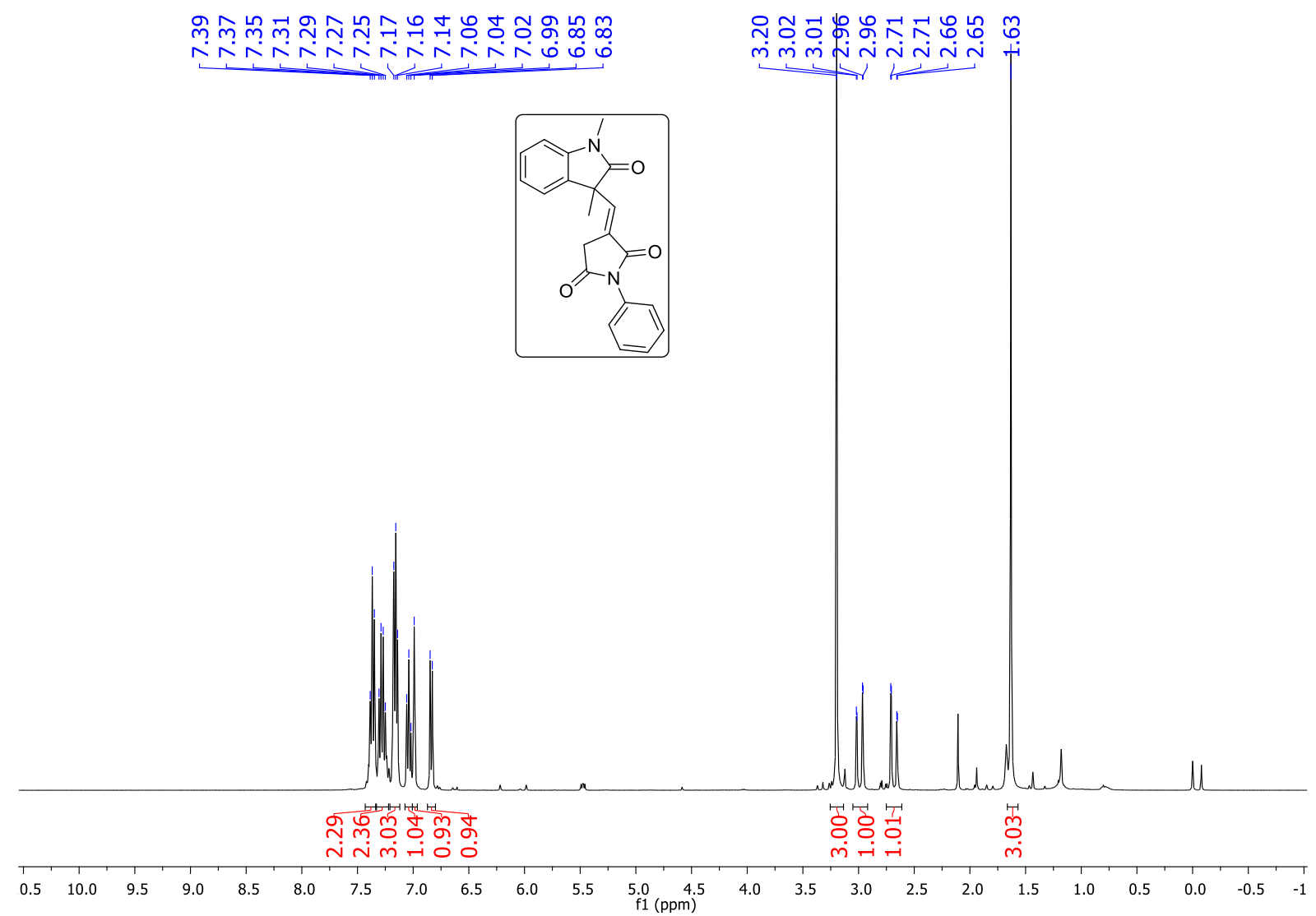

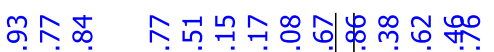

ம் ํำ

1,

苞 $\quad \stackrel{\infty}{\infty}$

के $\quad \vec{m} \stackrel{i}{\sim}$

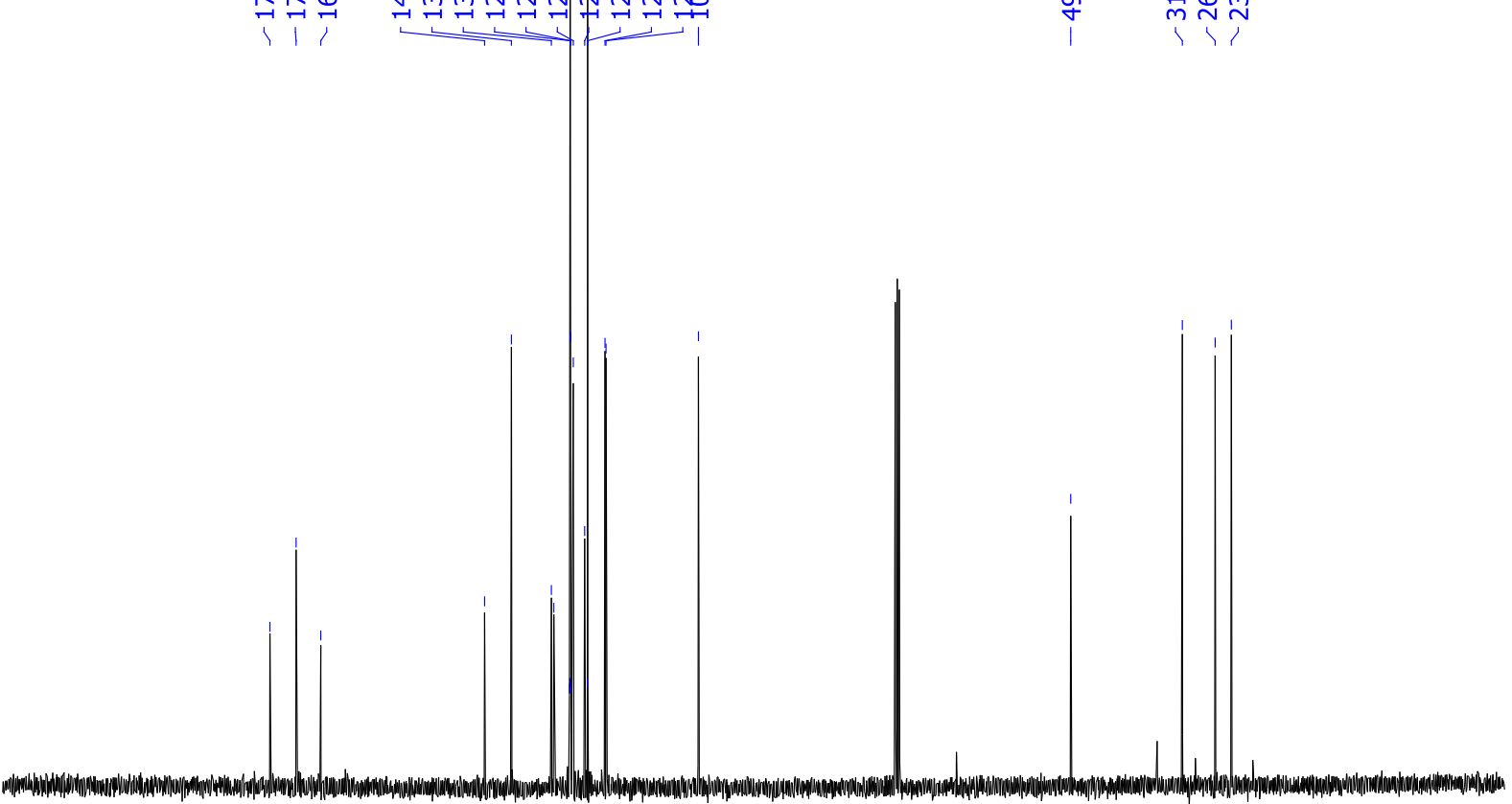

$\begin{array}{lllllllllllllllllllllll}210 & 200 & 190 & 180 & 170 & 160 & 150 & 140 & 130 & 120 & 110 & 100 & 90 & 80 & 70 & 60 & 50 & 40 & 30 & 20 & 10 & 0 & -10\end{array}$ 

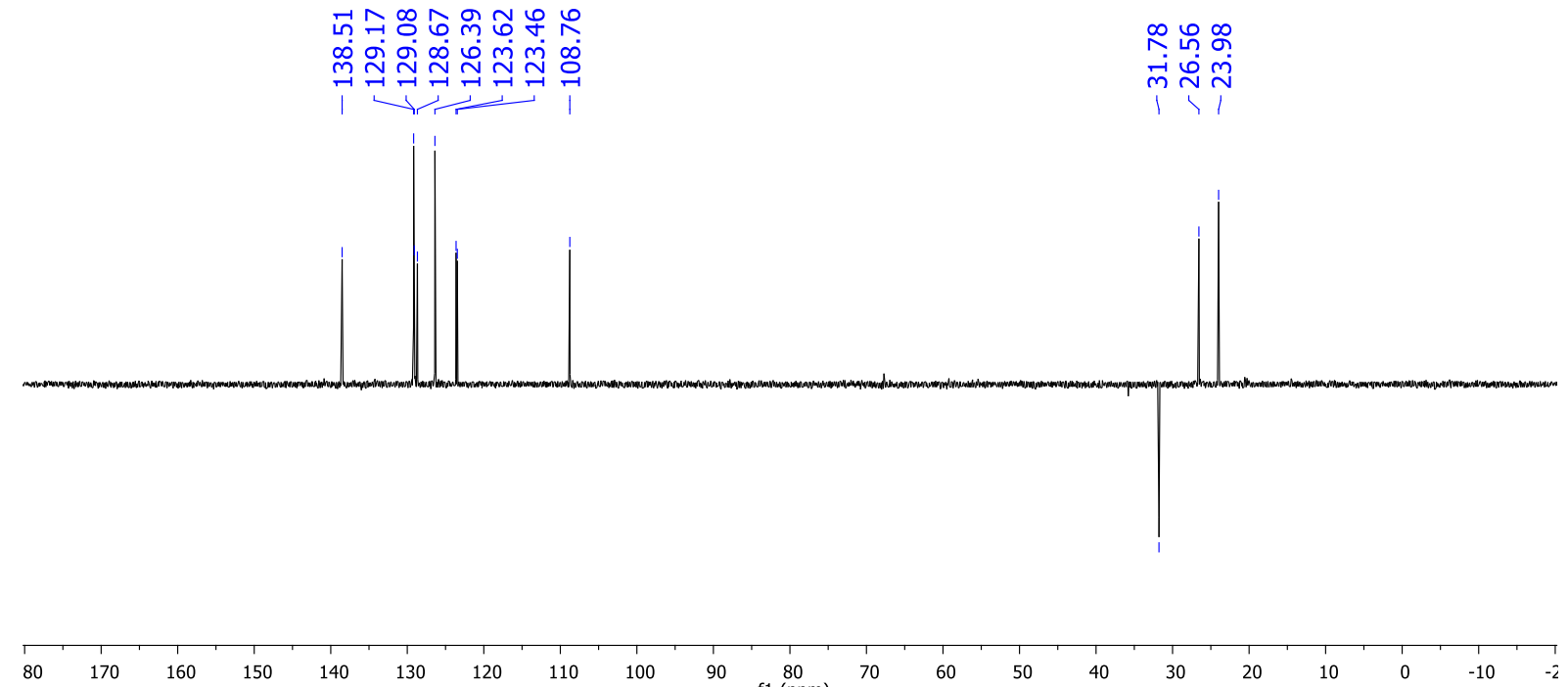
Copies of $\mathrm{H}^{1}, \mathrm{C}^{13}$ and DEPT 135 NMR spectra of compound 3af

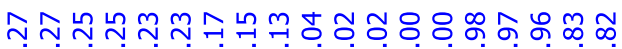

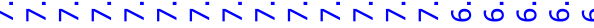

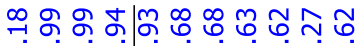

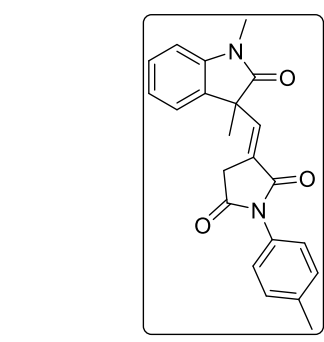
miñininini

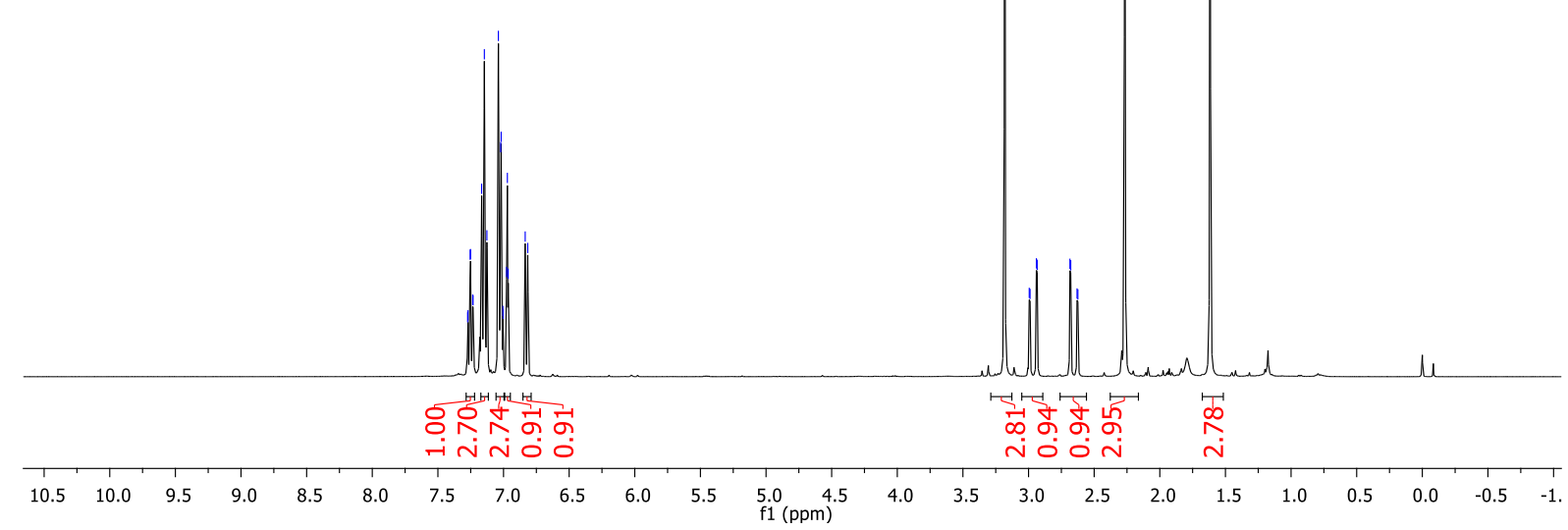

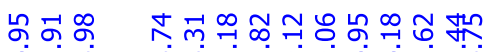

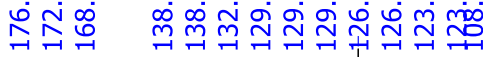

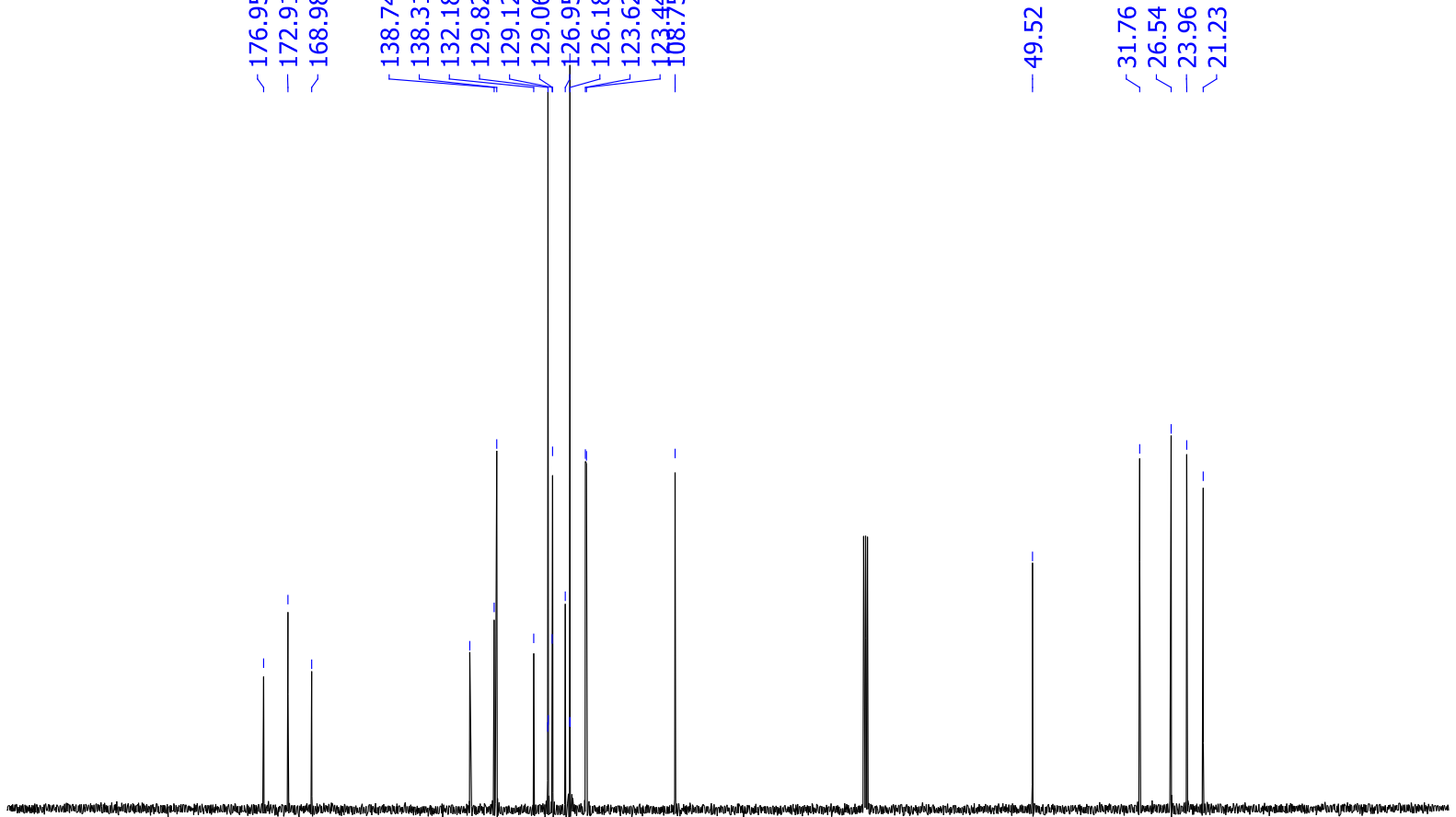

$\begin{array}{lllllllllllllllllllllllllll}210 & 200 & 190 & 180 & 170 & 160 & 150 & 140 & 130 & 120 & 110 & \begin{array}{l}100 \\ 100\end{array} & 90 & 80 & 70 & 60 & 50 & 40 & 30 & 20 & 10 & 0 & -10\end{array}$ 


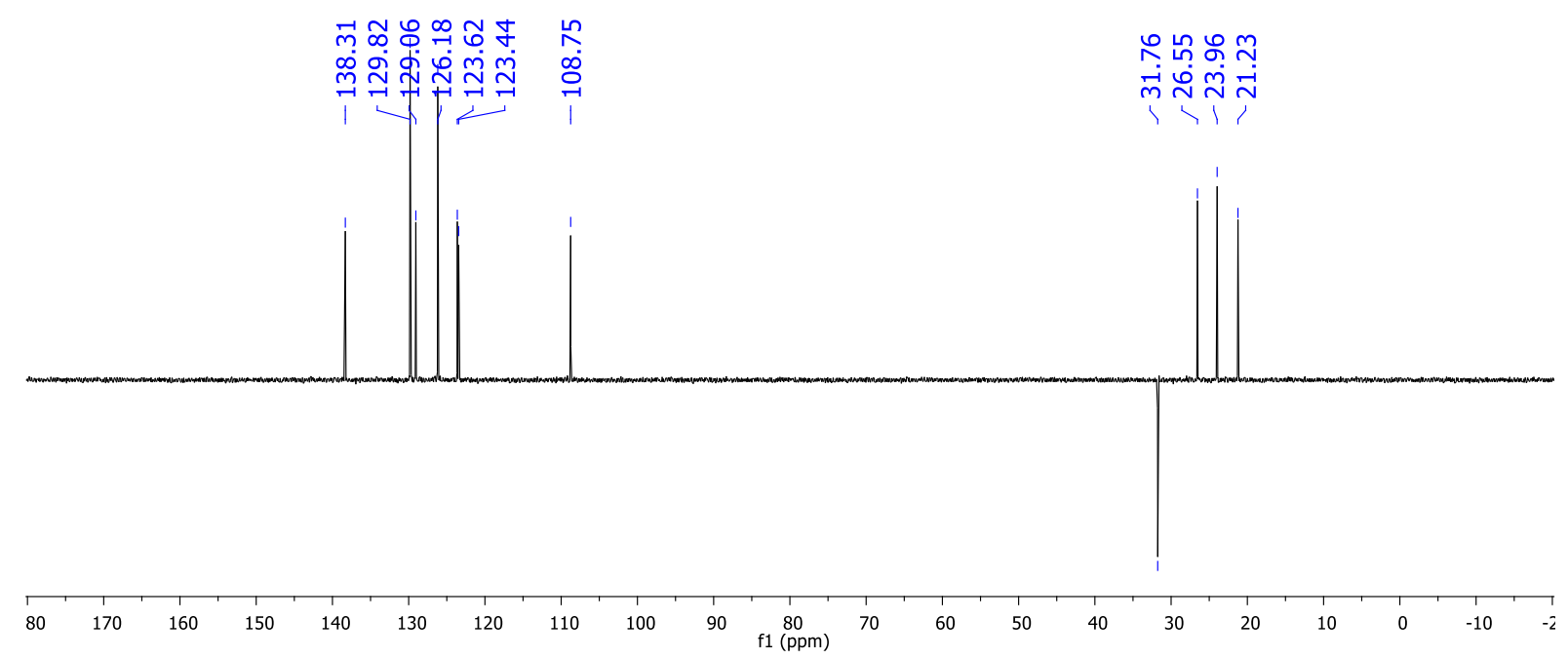


Copies of $\mathrm{H}^{1}, \mathrm{C}^{13}$ and DEPT 135 NMR spectra of compound 3ag
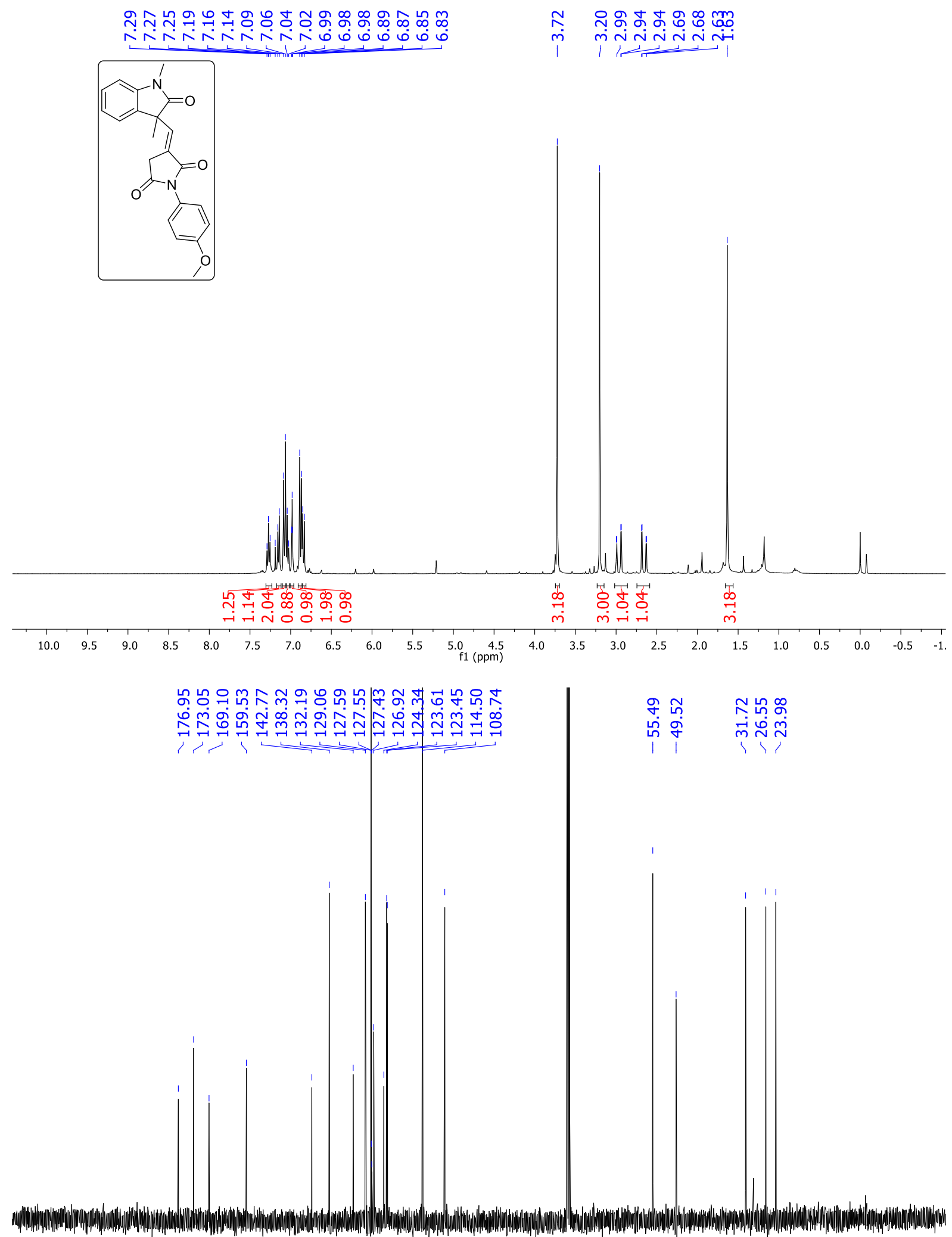

$\begin{array}{llllllllllllllllllllllll}210 & 200 & 190 & 180 & 170 & 160 & 150 & 140 & 130 & 120 & 110 & \underset{\mathrm{f} 1}{100}(\mathrm{ppm}) & 90 & 80 & 70 & 60 & 50 & 40 & 30 & 20 & 10 & 0 & -10\end{array}$ 


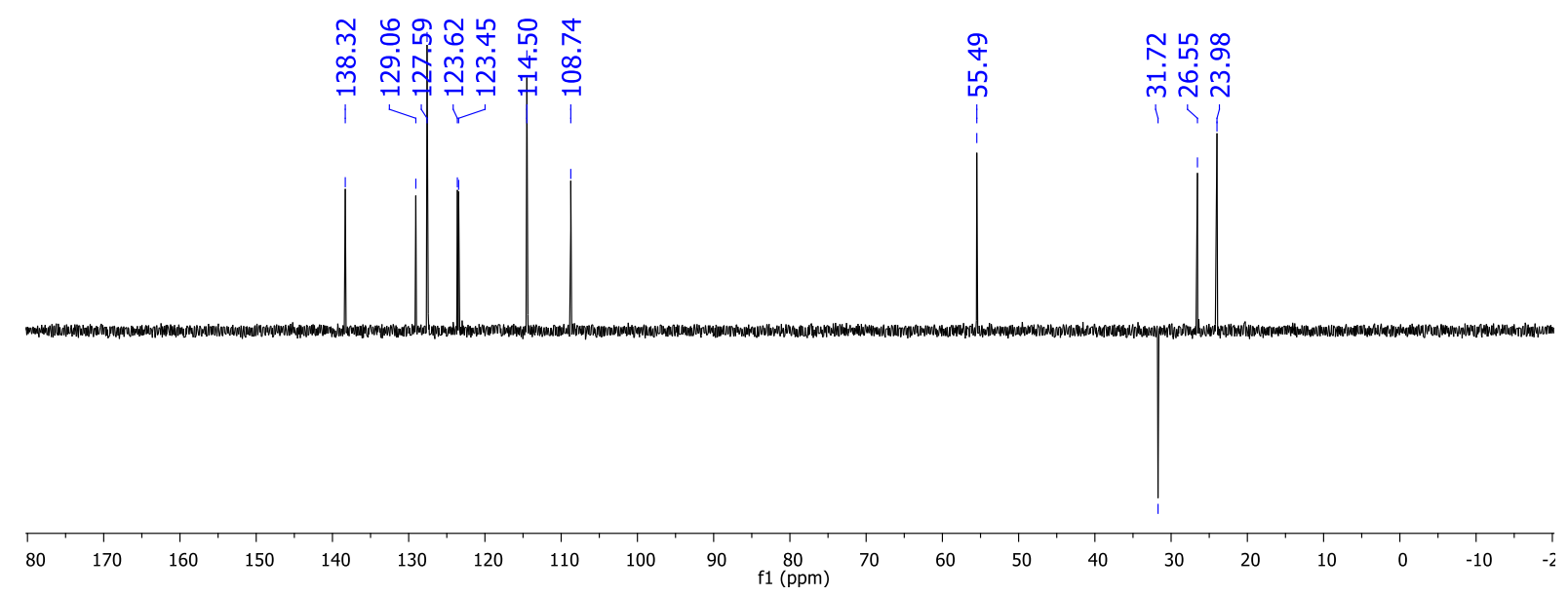


Copies of $\mathrm{H}^{1}, \mathrm{C}^{13}$ and DEPT 135 NMR spectra of compound 3ah

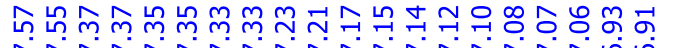

ÑNNNNNNNN

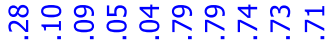

(1) m.minininis

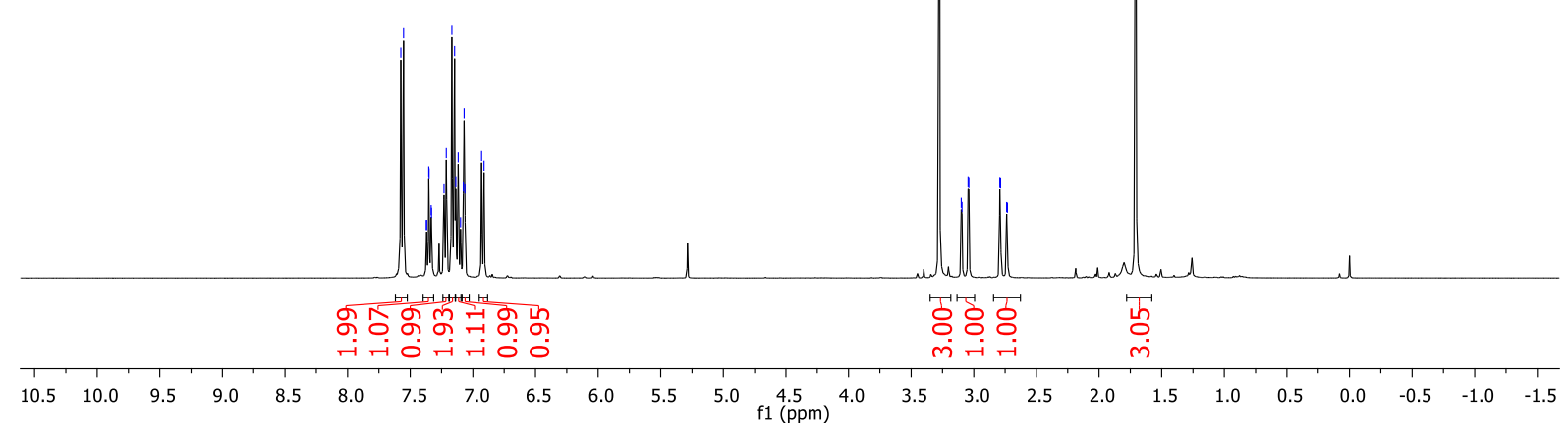

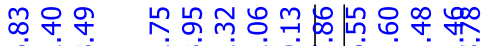

ปั

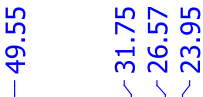

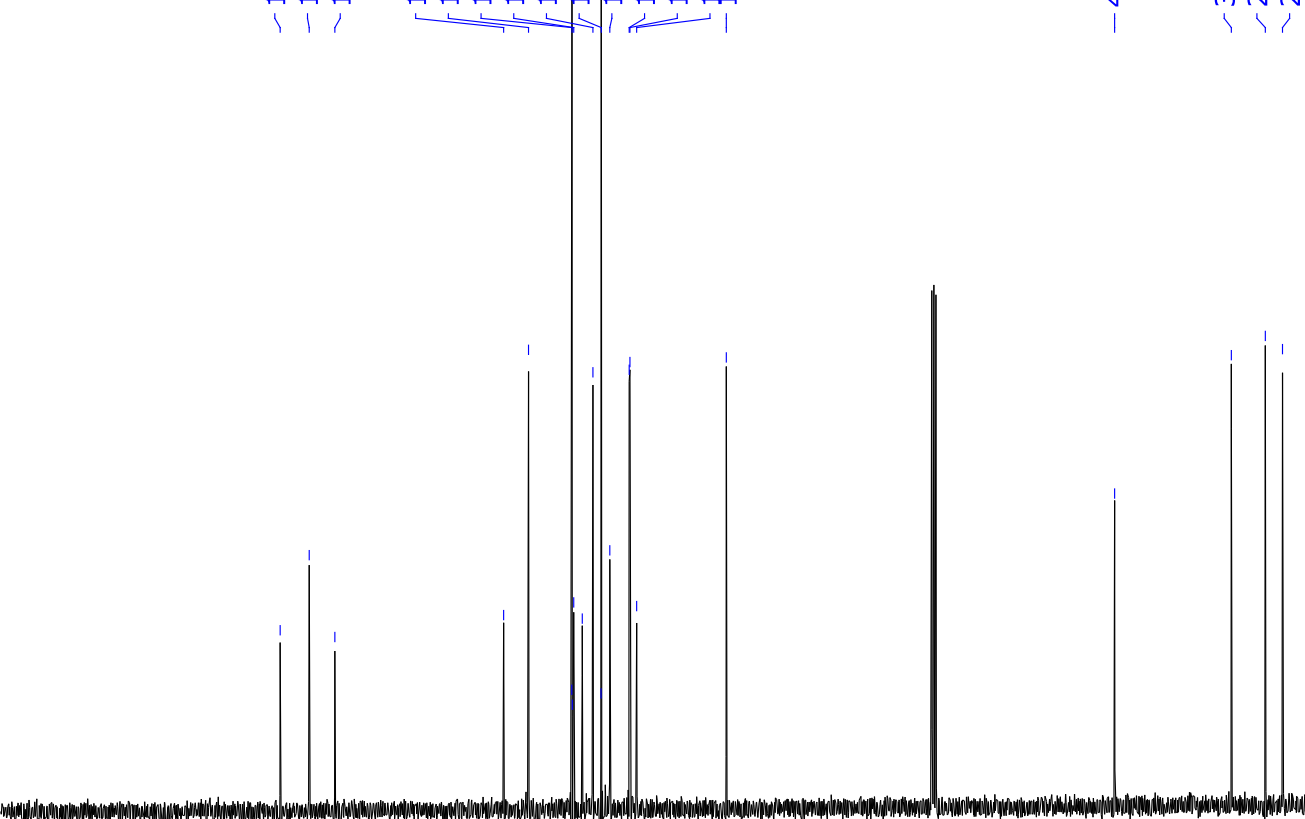

$\begin{array}{lllllllllllllllllllllll}210 & 200 & 190 & 180 & 170 & 160 & 150 & 140 & 130 & 120 & 110 & 100 & 90 & 80 & 70 & 60 & 50 & 40 & 30 & 20 & 10 & 0 & -10\end{array}$ 


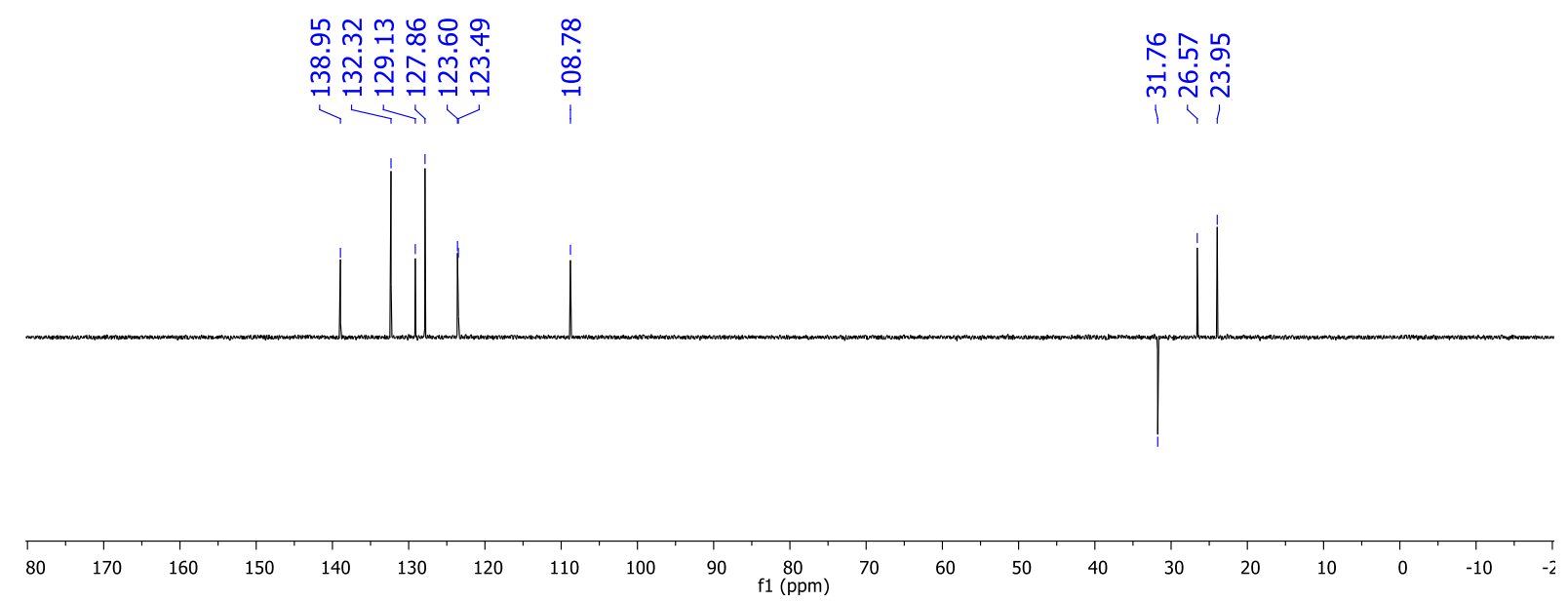


Copies of $\mathrm{H}^{1}, \mathrm{C}^{13}$ and DEPT 135 NMR spectra of compound 5aa
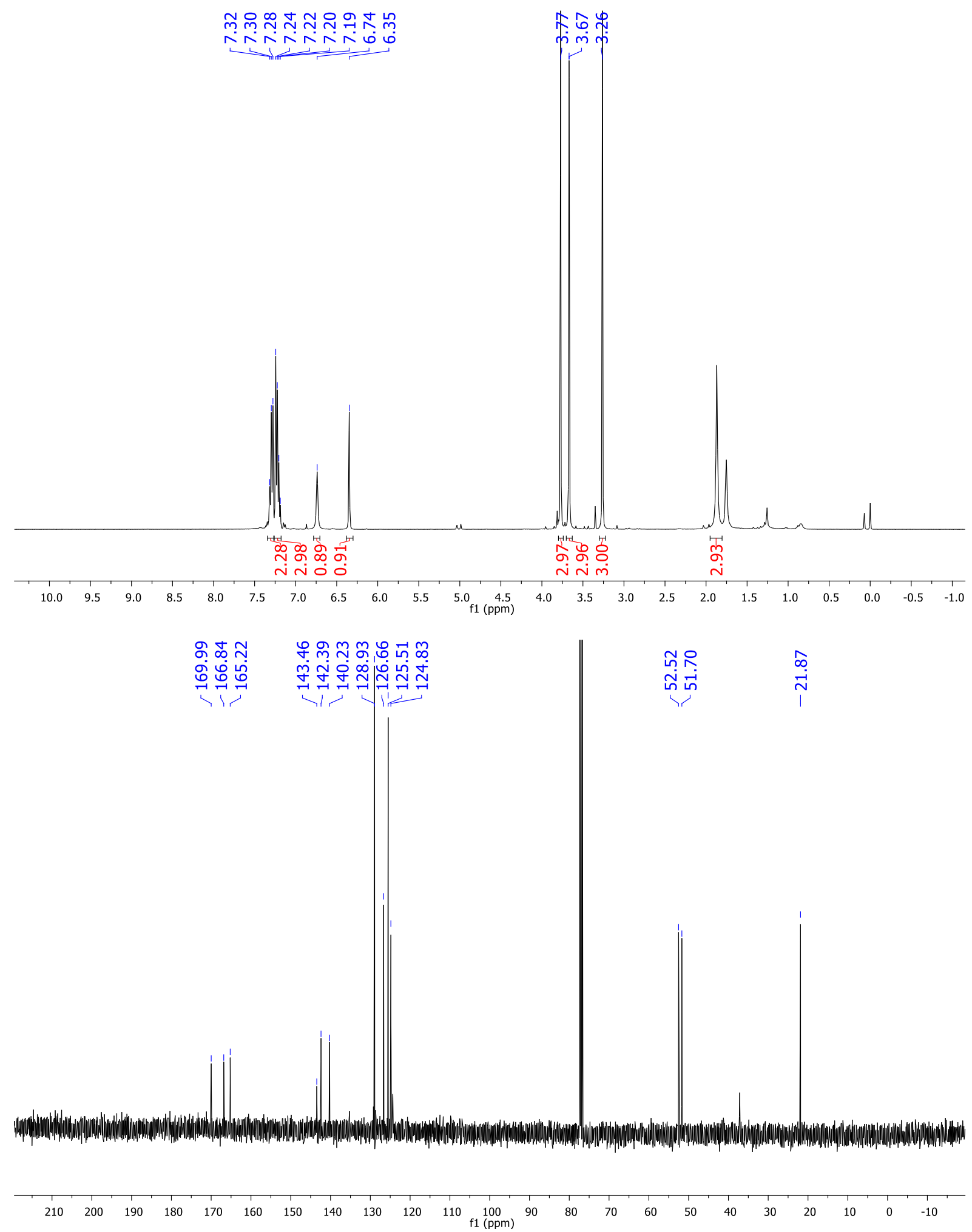


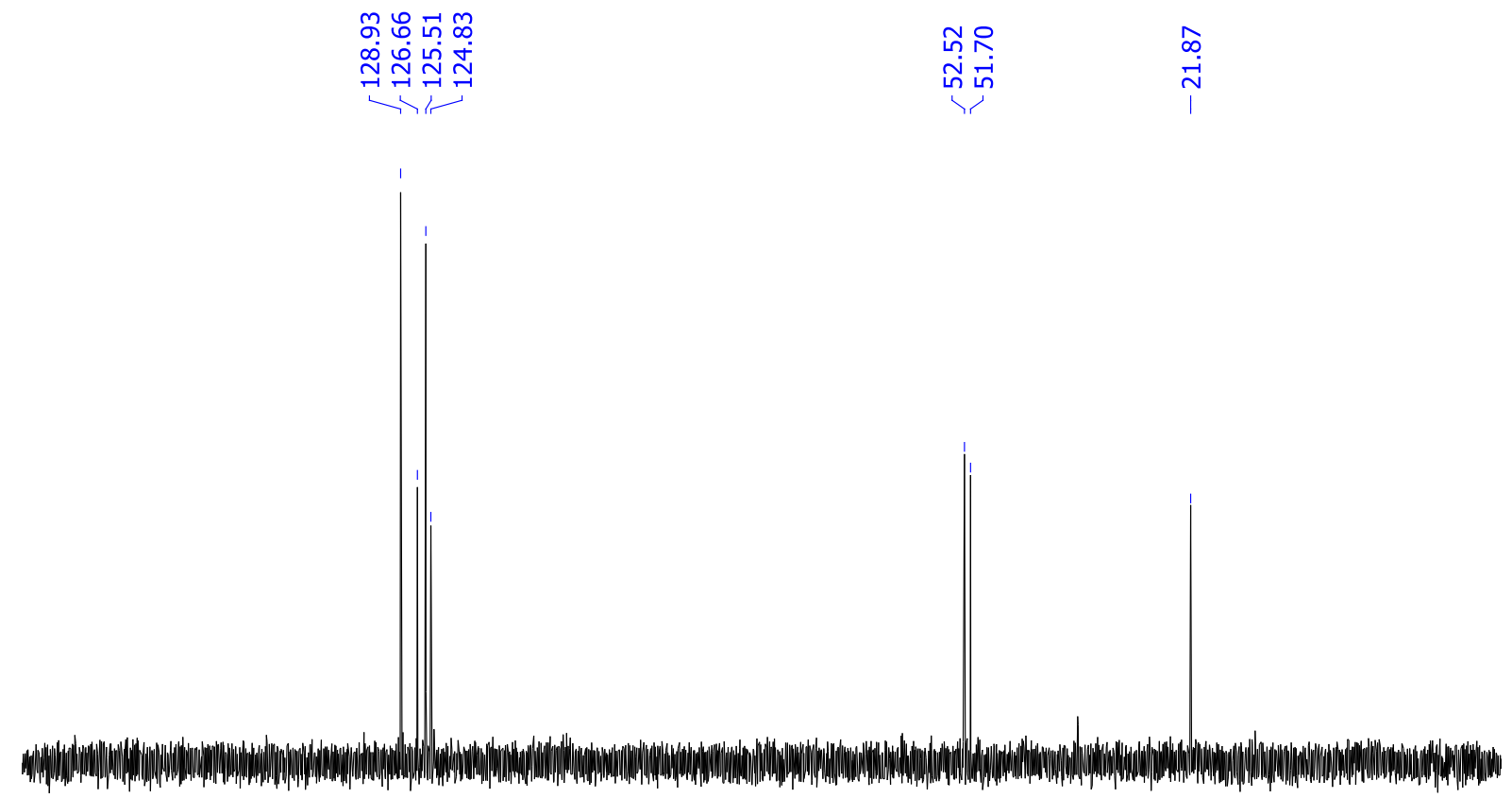

\begin{tabular}{lllllllllllllllllllll}
\hline 80 & 170 & 160 & 150 & 140 & 130 & 120 & 110 & 100 & 90 & $\begin{array}{c}80 \\
\mathrm{f} 1(\mathrm{ppm})\end{array}$ & 70 & 60 & 50 & 40 & 30 & 20 & 10 & 0 & -10 & -2
\end{tabular}

Copy of NOESY Experiment of Compound 5aa

There is no NOE correlation between Ha 6.35 (s, 1H) and methyl groups Hb $6.74(\mathrm{~s}, 1 \mathrm{H})$. These results clearly revealed that the regiochemistry of compound 5aa is correct.

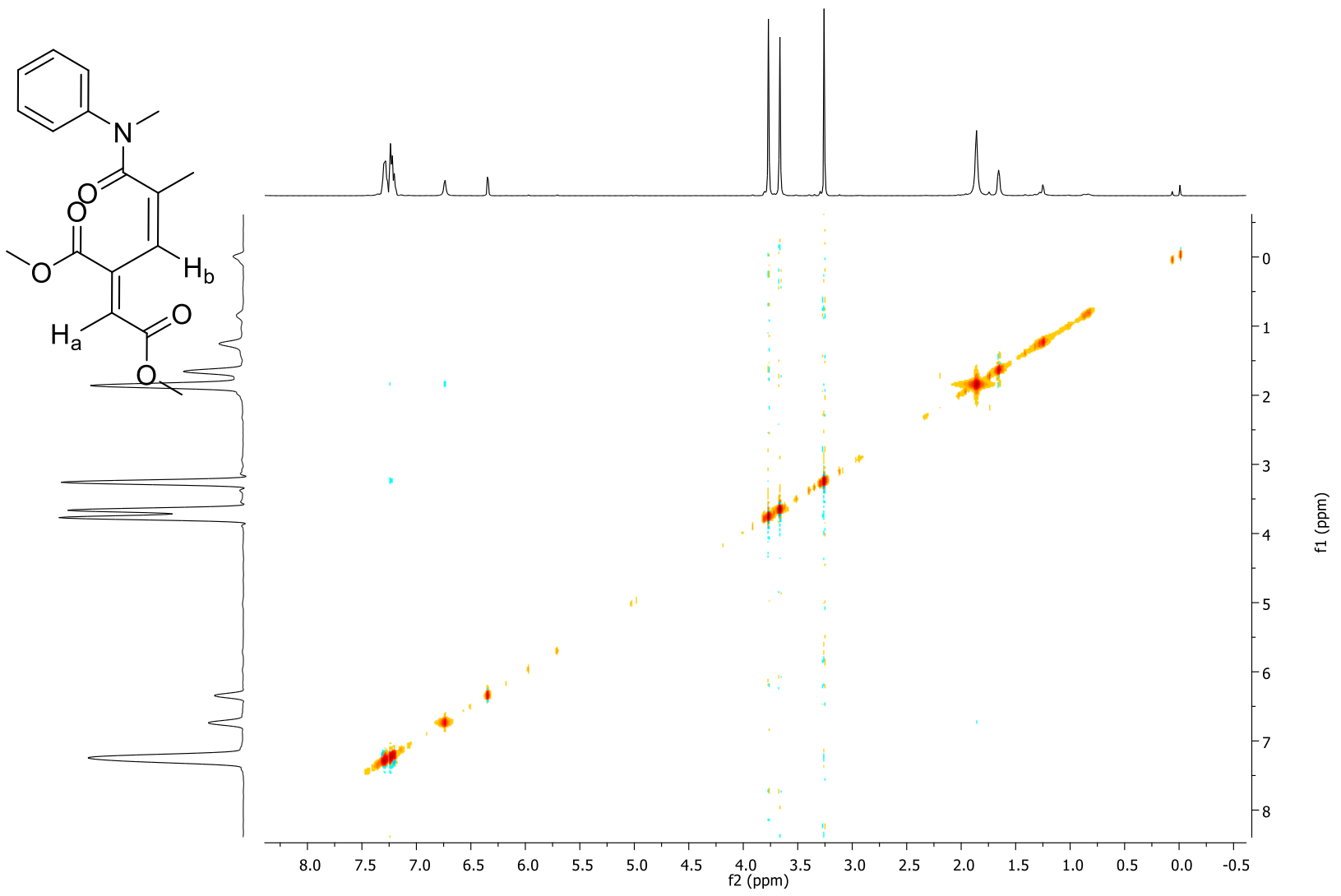


Copies of $\mathrm{H}^{1}, \mathrm{C}^{13}$ and DEPT $135 \mathrm{NMR}$ spectra of compound 5aa'
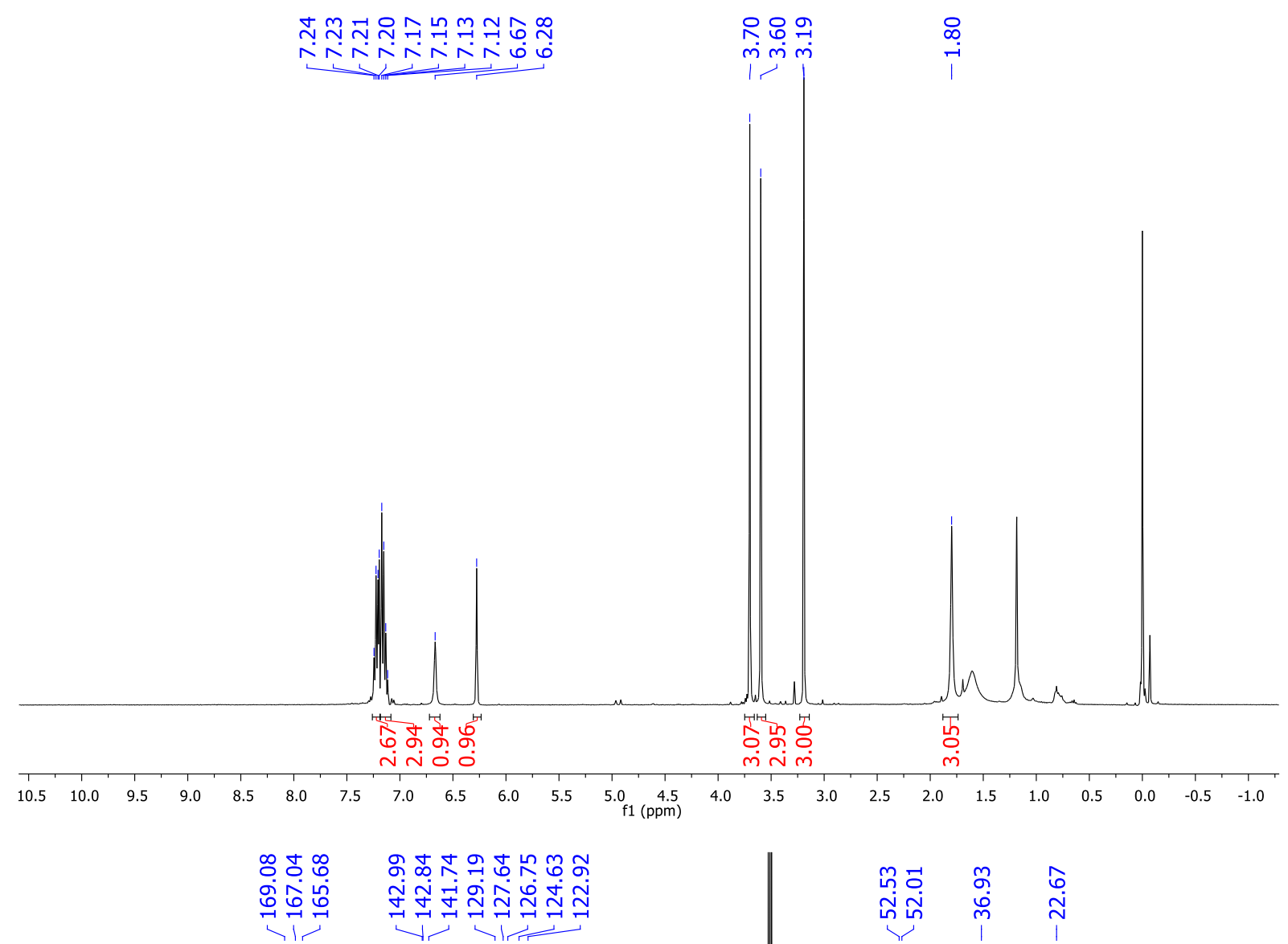

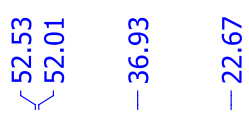

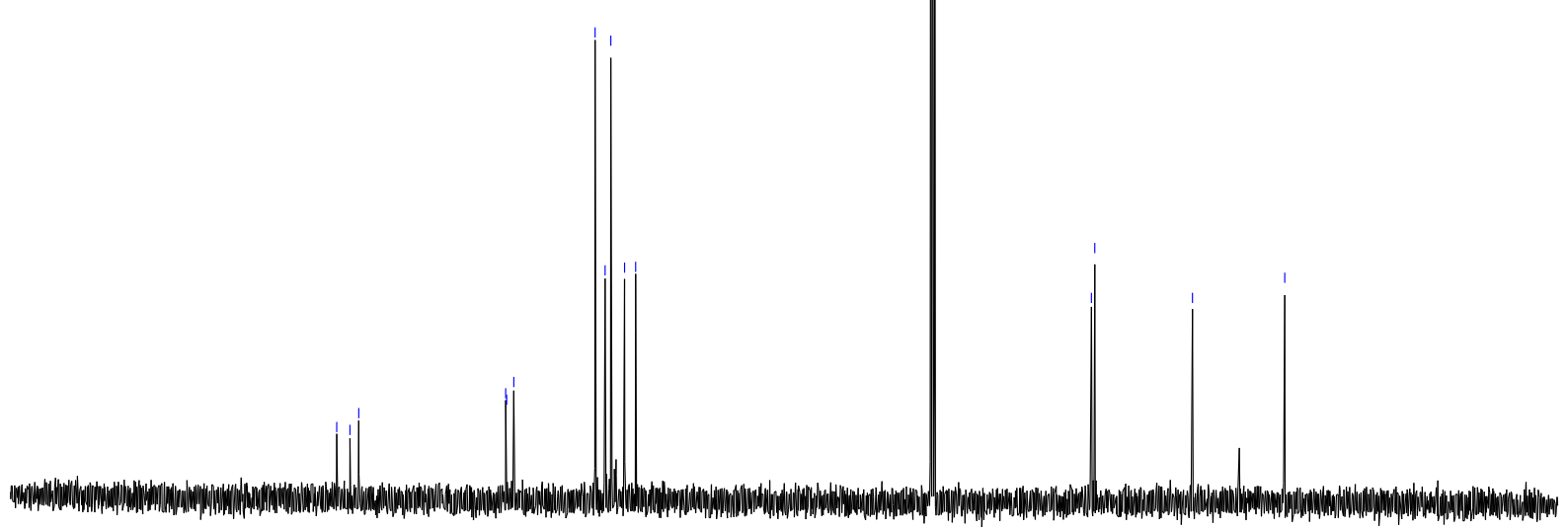

$\begin{array}{llllllllllllllllllllllll}210 & 200 & 190 & 180 & 170 & 160 & 150 & 140 & 130 & 120 & 110 & 100 & 90 & 80 & 70 & 60 & 50 & 40 & 30 & 20 & 10 & 0 & -10\end{array}$ 


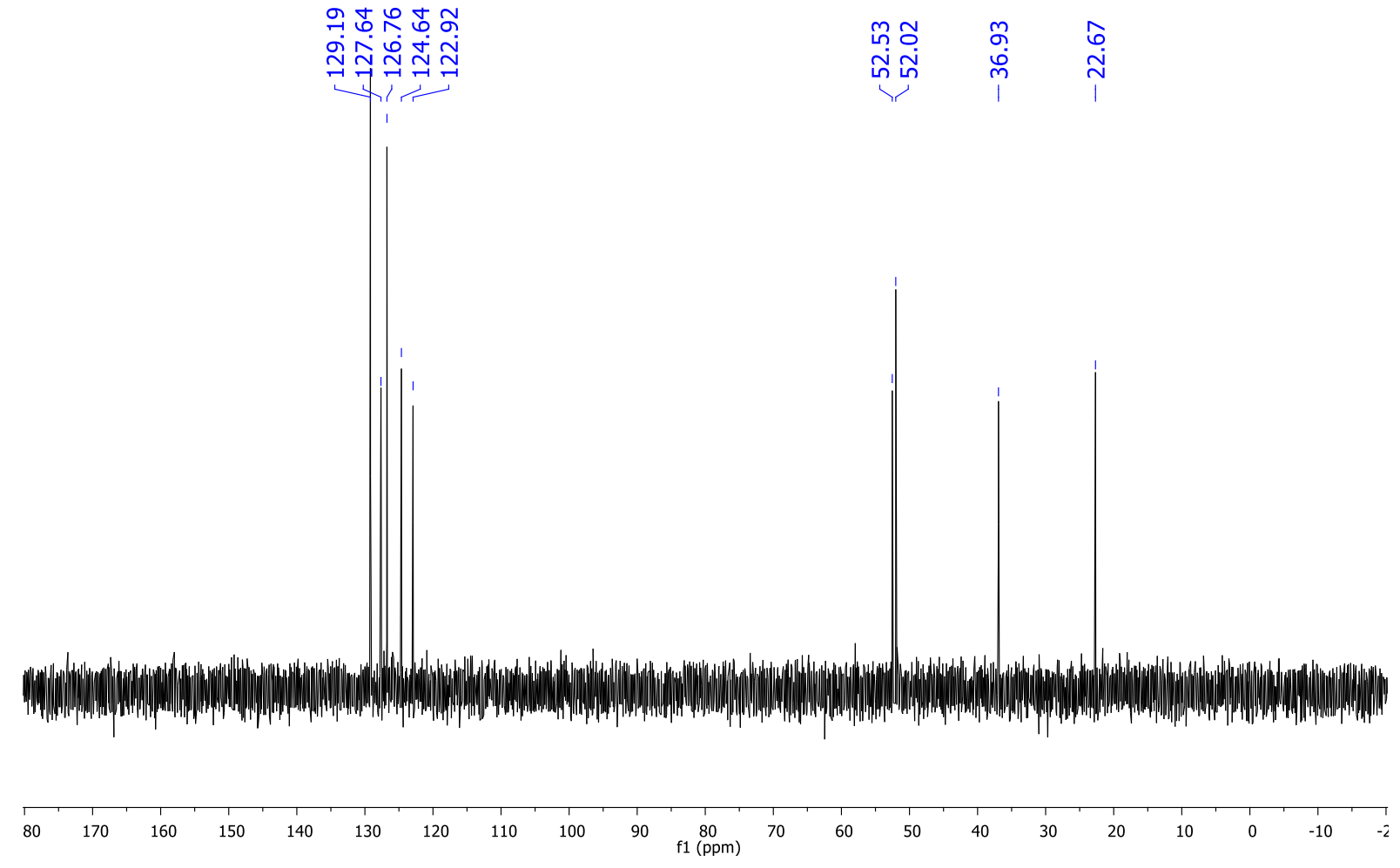

Copy of NOESY Experiment of Compound 5aa'

There is a NOE correlation between Ha $5.70(\mathrm{~s}, 1 \mathrm{H})$ and methyl groups $\mathrm{Hb} 5.98(\mathrm{~s}, 1 \mathrm{H})$.

These results clearly revealed that the regiochemistry of compound 5aa' is correct.

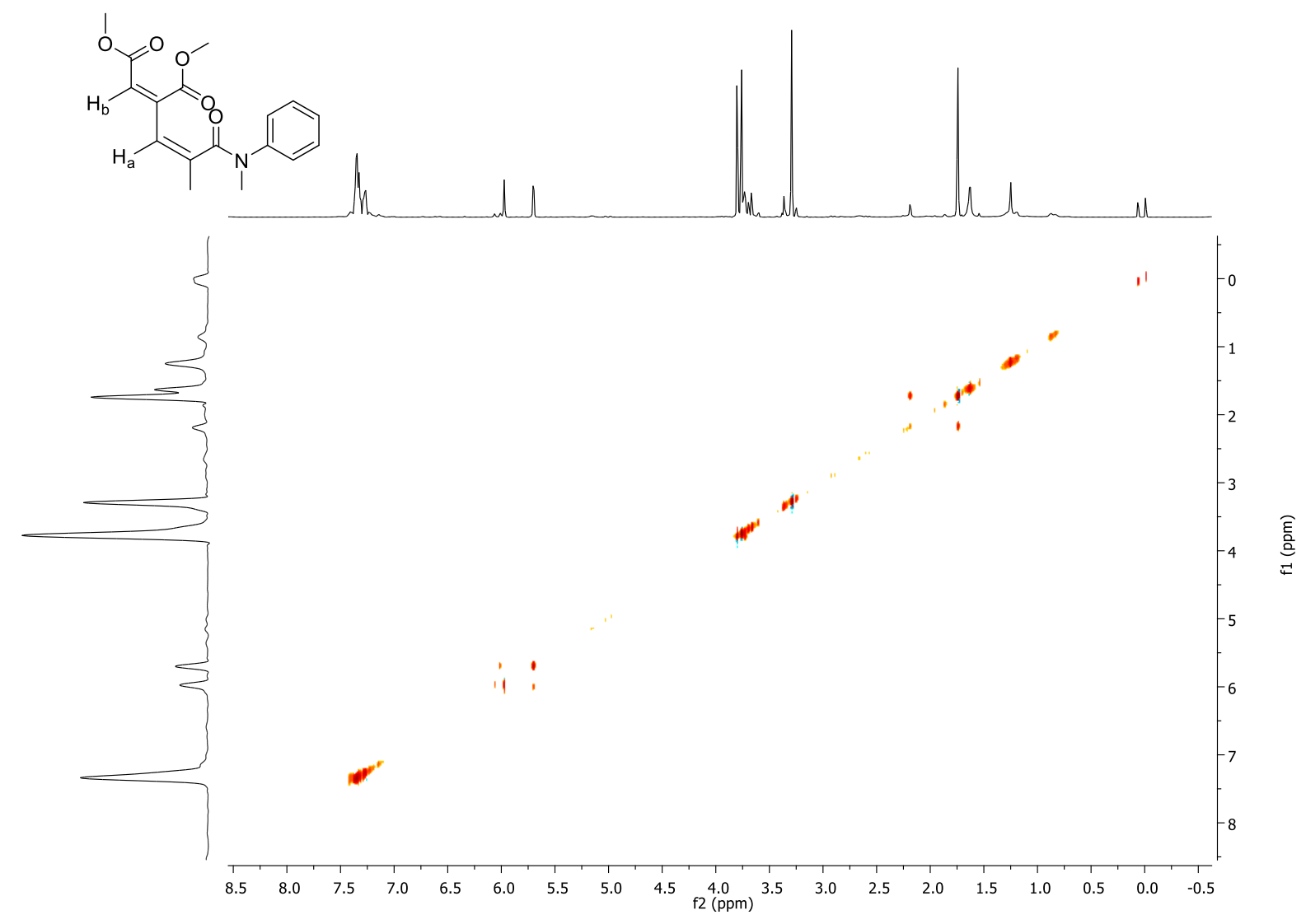


Copies of $\mathrm{H}^{1}, \mathrm{C}^{13}$ and DEPT 135 NMR spectra of compound 5ab
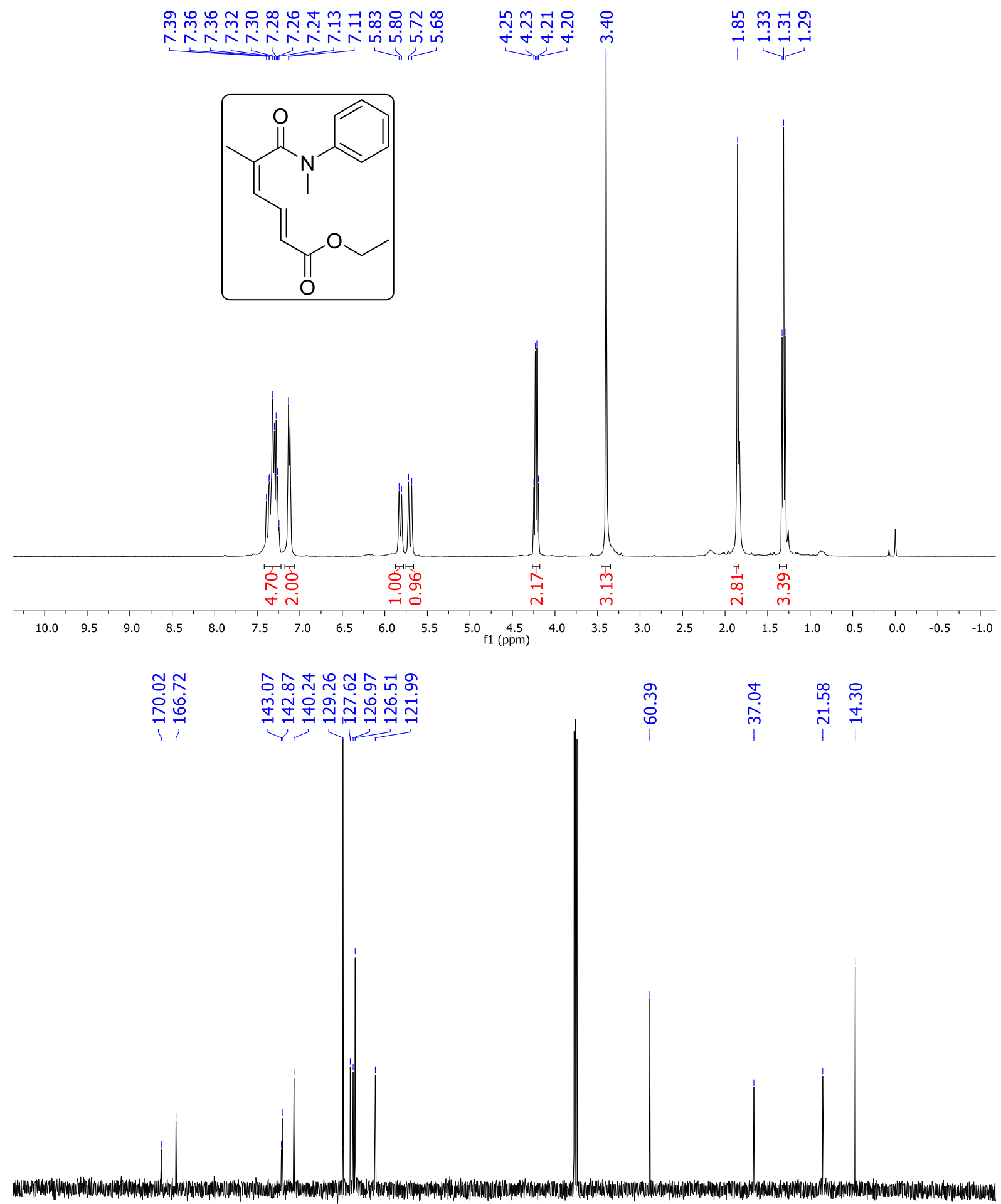

$\begin{array}{lllllllllll}200 & 190 & 180 & 170 & 160 & 150 & 140 & 130 & 120 & 110 & 100 \begin{array}{r}90 \\ f 1(\mathrm{ppm})\end{array}\end{array}$ 㽞 นุ̣กㄲำ

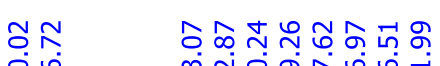

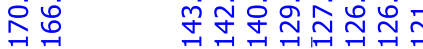

80

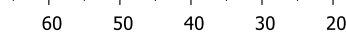

$\begin{array}{lll}10 & 0 & -10\end{array}$ 


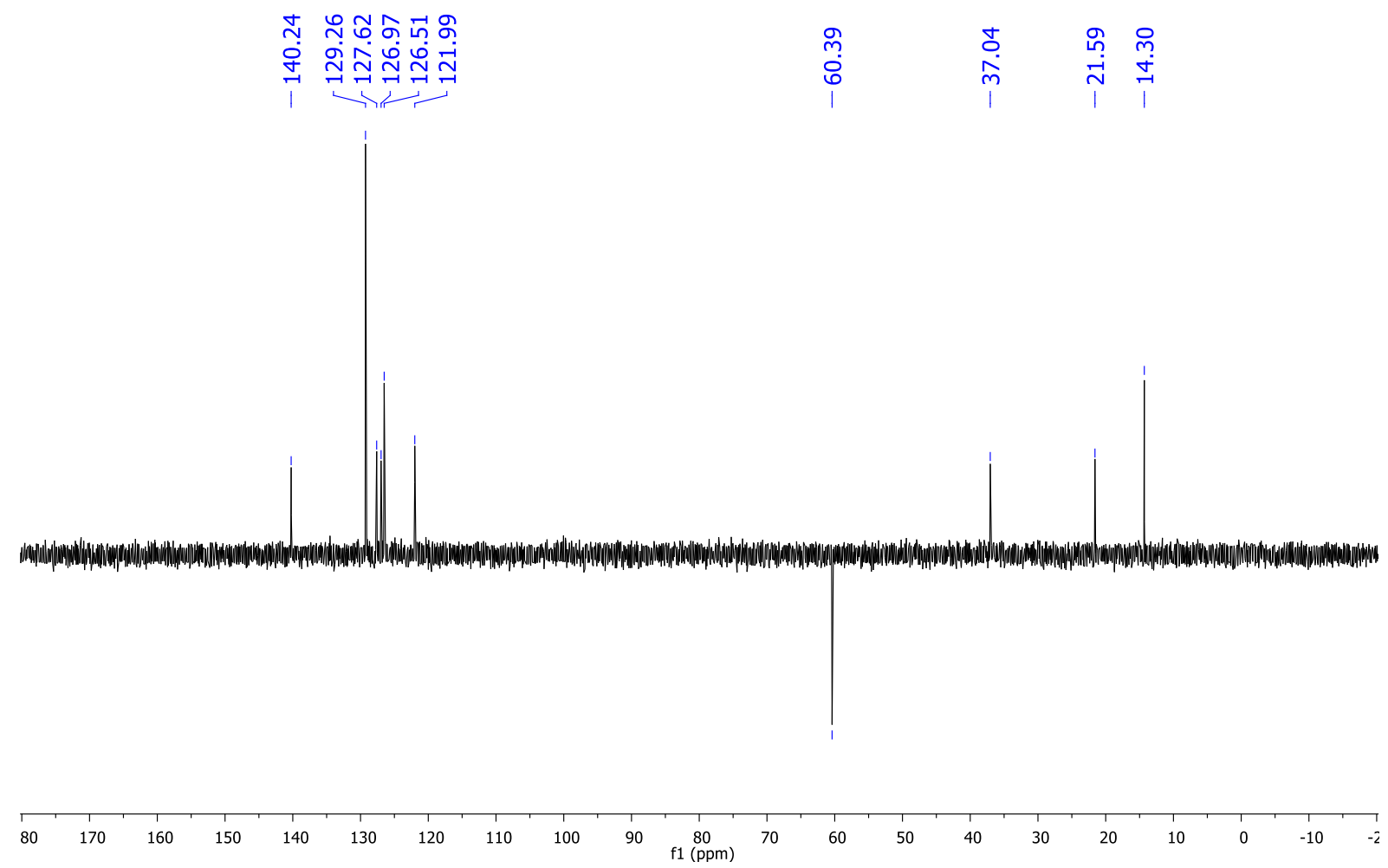


Copies of $\mathrm{H}^{1}, \mathrm{C}^{13}$ and DEPT 135 NMR spectra of compound 5ac

œ

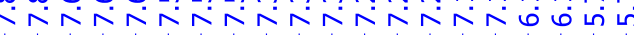
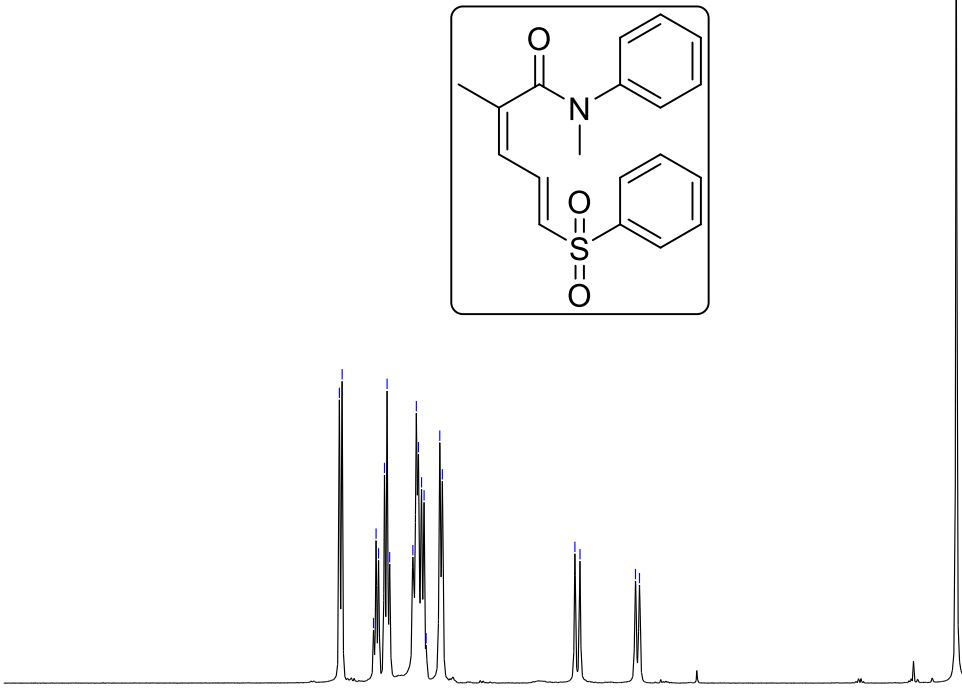

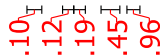

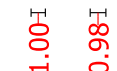

$\begin{array}{lllllllllll}10.0 & 9.5 & 9.0 & 8.5 & 8.0 & 7.5 & 7.0 & 6.5 & 6.0 & 5.5 & 5.0 \\ \mathrm{f} 1(\mathrm{ppm}) & 4.5\end{array}$

กิ సู

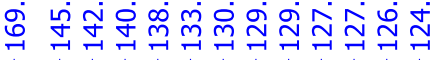

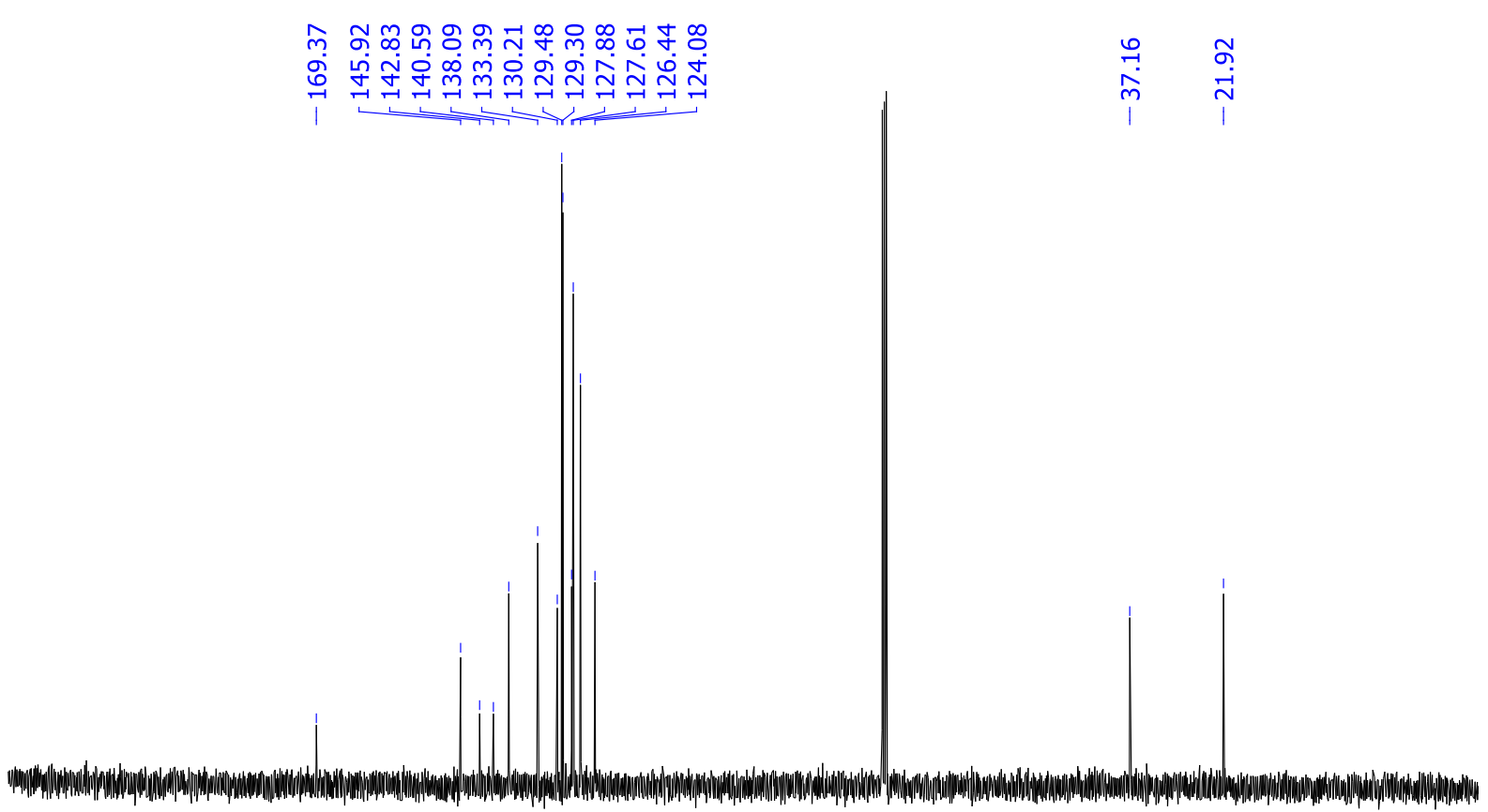

$\begin{array}{llllllllllllllllllllllllllll}210 & 200 & 190 & 180 & 170 & 160 & 150 & 140 & 130 & 120 & 110 & \underset{\mathrm{f} 1(\mathrm{ppm})}{100} & 90 & 80 & 70 & 60 & 50 & 40 & 30 & 20 & 10 & 0 & -10\end{array}$

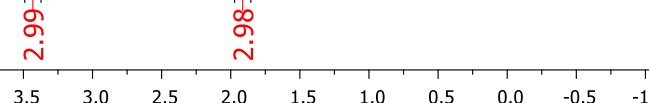




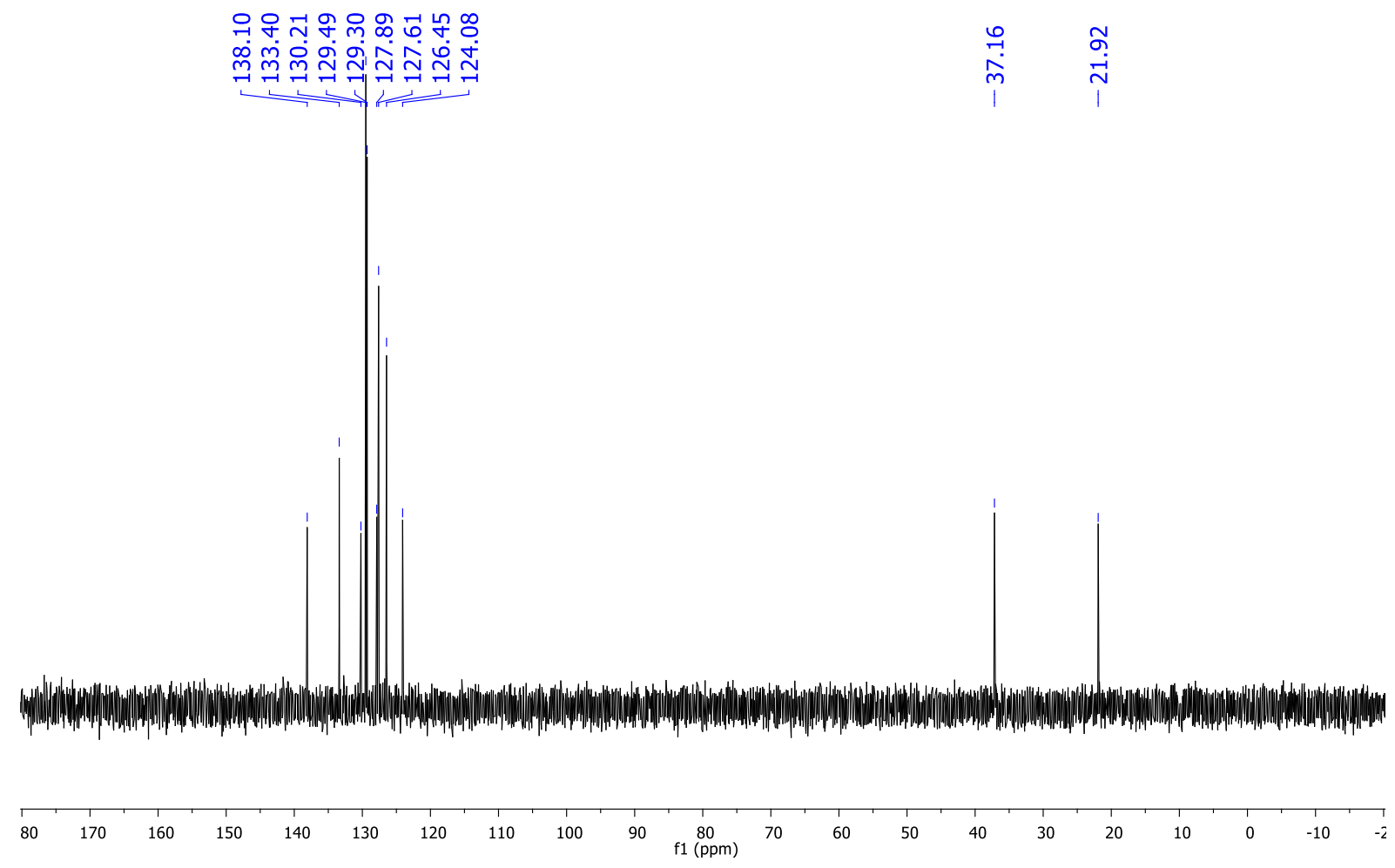


Copies of $\mathrm{H}^{1}, \mathrm{C}^{13}$ and DEPT 135 NMR spectra of compound 6
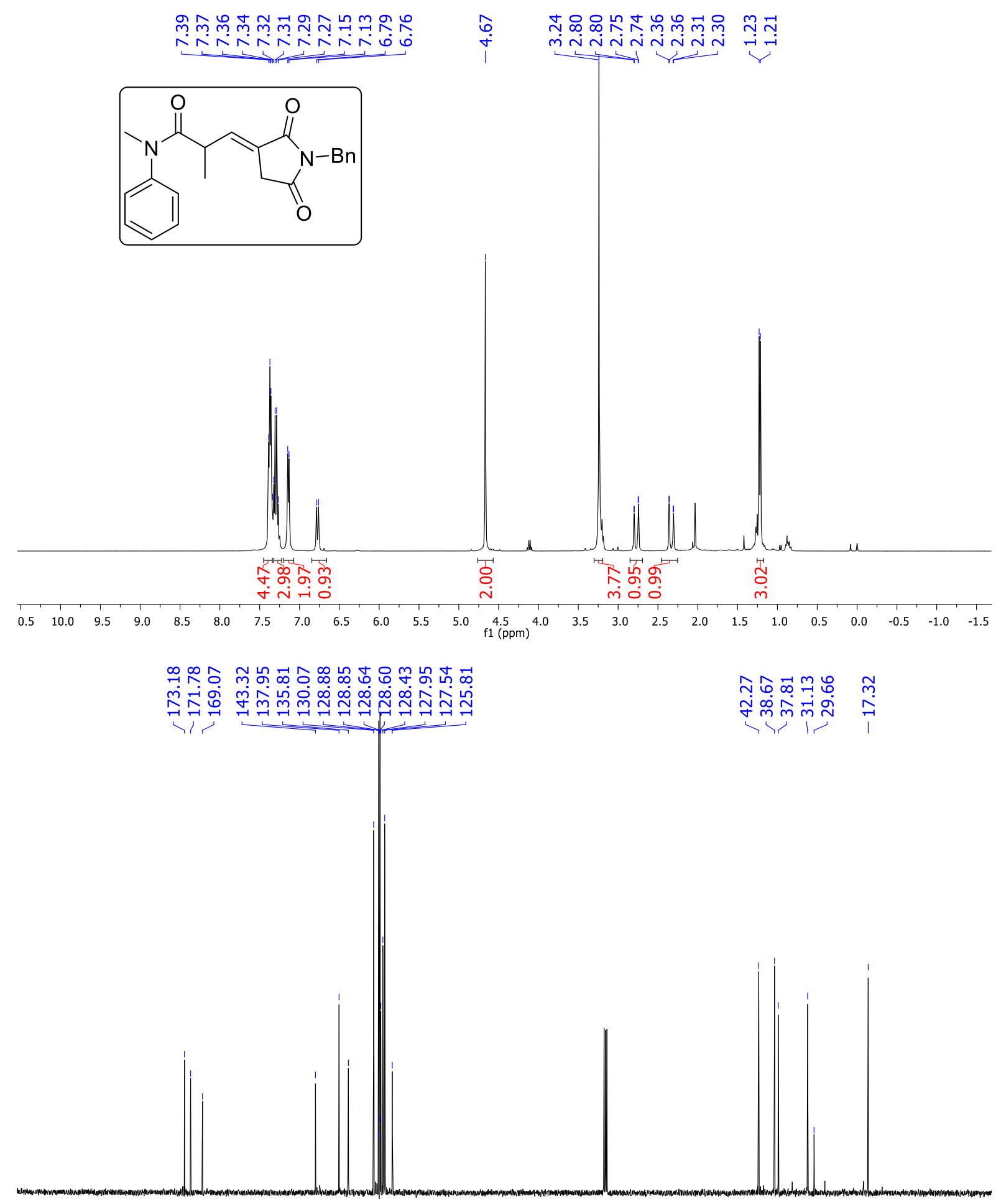

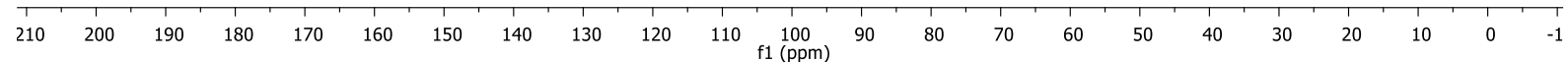




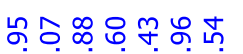

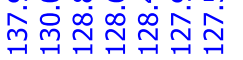

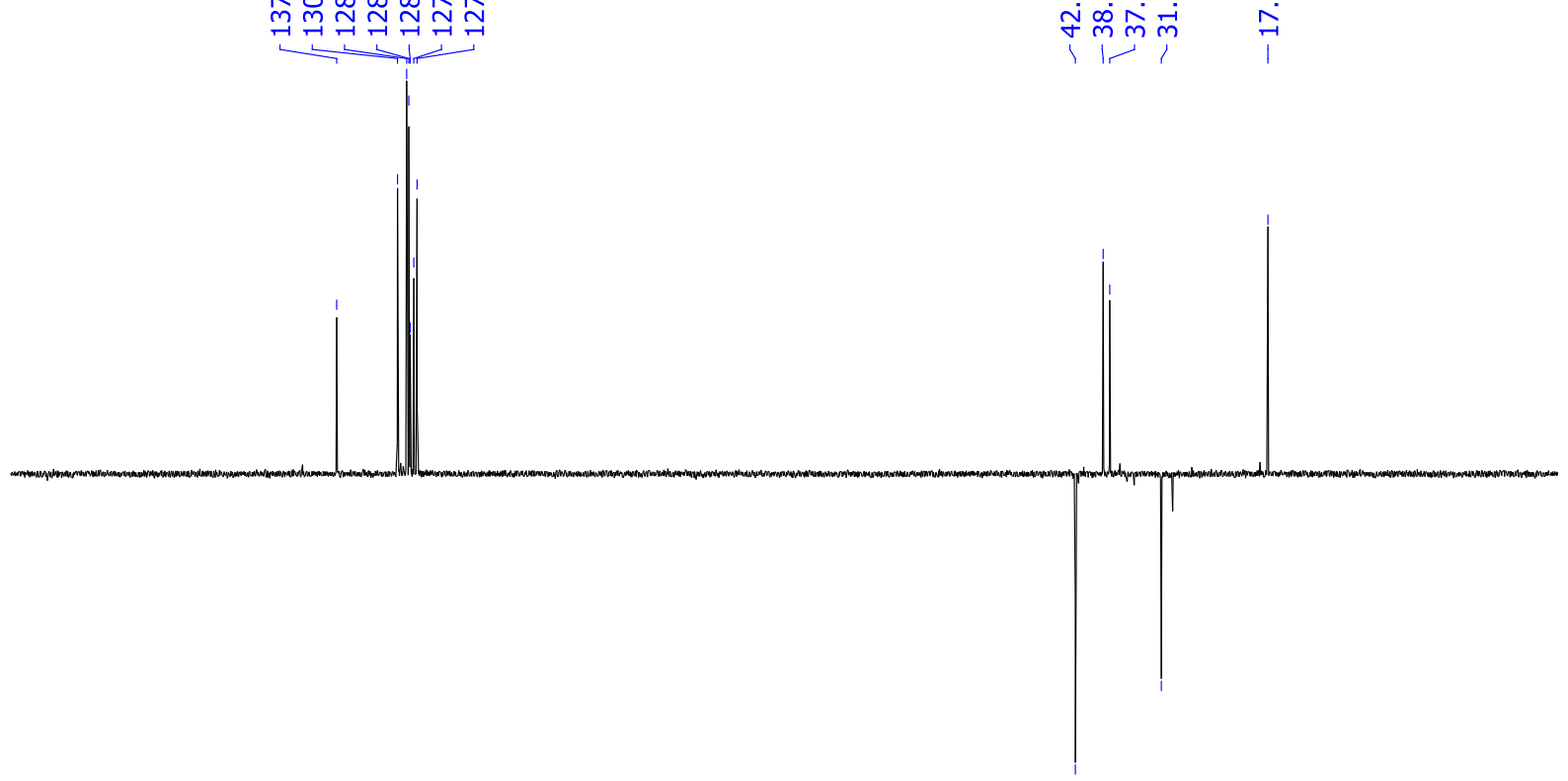

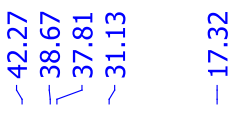

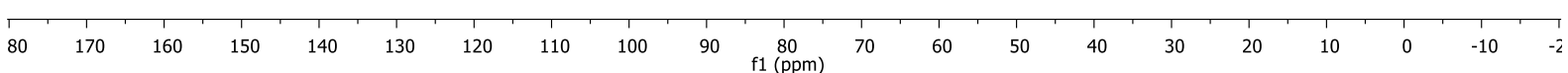

